$$
\begin{gathered}
\text { Aus dem Fachbereich Medizin } \\
\text { der Johann Wolfgang Goethe-Universität } \\
\text { Frankfurt am Main }
\end{gathered}
$$

\author{
betreut am \\ Zentrum der Radiologie \\ Institut für Diagnostische und Interventionelle Radiologie \\ Direktor: Prof. Dr. Thomas Vogl
}

\title{
Diagnostik von Bandscheibenvorfällen der Lendenwirbelsäule mittels virtueller Noncalcium Dual-Energy-Computertomographie
}

\author{
Dissertation \\ zur Erlangung des Doktorgrades der Medizin \\ des Fachbereichs Medizin \\ der Johann Wolfgang Goethe-Universität \\ Frankfurt am Main
}

vorgelegt von

Jochen Gotthard Günter Nöske

aus Annweiler am Trifels

Frankfurt am Main, 2021 
Dekan:

Referent:

Korreferent/in:

Tag der mündlichen Prüfung:
Prof. Dr. Stefan Zeuzem

Prof. Dr. Thomas Vogl

Prof. Dr. Andrea Meurer

15. Oktober 2021 


\title{
Diagnostik von Bandscheibenvorfällen der Lendenwirbelsäule mittels virtueller Noncalcium Dual-Energy-Computertomographie
}

\author{
Diagnosis of Lumbar Disk Herniation using virtual Noncalcium \\ Dual-Energy Computed Tomography
}

Teile dieser Arbeit wurden publiziert in:

Parts of this thesis were published in:

(1) Booz C, Nöske J, Martin SS, Albrecht MH, Yel I, Lenga L, GruberRoth T, Eichler K, D'Angelo T, Vogl TJ, Wichmann JL Virtual Noncalcium Dual-Energy CT: Detection of Lumbar Disk Herniation in Comparison with Standard Grayscale CT. Radiology. 2019 Feb;290(2):446-455. doi: 10.1148/radiol.2018181286 
Inhaltsverzeichnis

1 Zusammenfassung ............................................................. 8

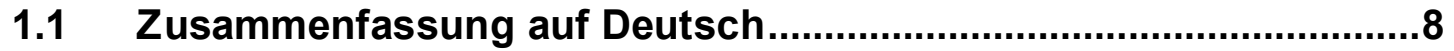

1.2 Abstract in English .................................................................10

2 Einführung ..................................................................... 12

2.1 Hintergrund und Zielsetzung dieser Studie ....................................12

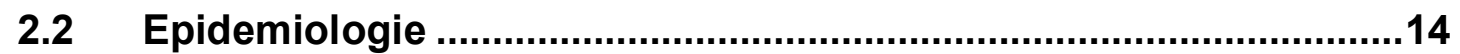

2.3 Aufbau und Funktion der Bandscheiben ......................................15

2.4 Pathologien der Bandscheiben .................................................17

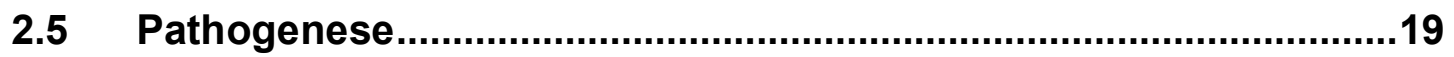

2.6 Klinische Symptomatik und Untersuchung ...................................22

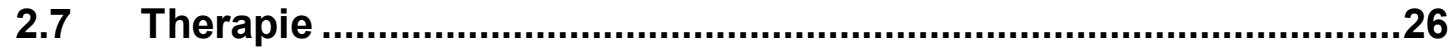

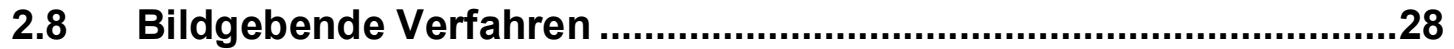

2.9 Dual-Energy-Computertomographie............................................32

2.9.1 Virtuelle Noncalcium Technik ........................................................38

3 Material und Methoden...................................................... 40

3.1 Patientenselektion und Studiendesign ........................................40

3.2 DECT Untersuchungsprotokoll ...................................................43

3.3 MRT Untersuchungsprotokoll.....................................................45

3.4 CT-Bildrekonstruktion und Nachverarbeitung .............................46

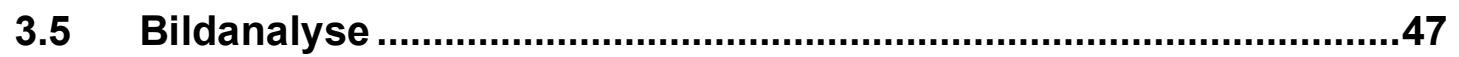

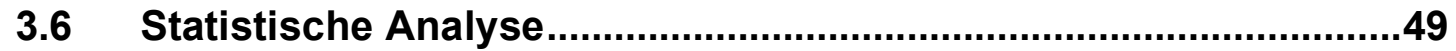

4 Ergebnisse ......................................................................... 51

4.1 Diagnostische Genauigkeit pro Patient........................................51

4.2 Diagnostische Genauigkeit pro Bandscheibe ..............................55

4.3 Qualitative Bewertung der Studienparameter ..............................62

5 Diskussion ........................................................................... 66

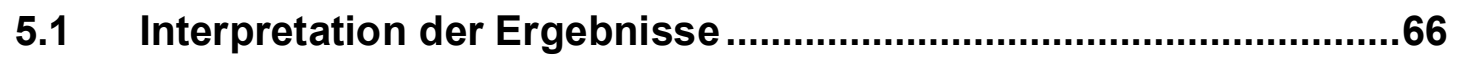

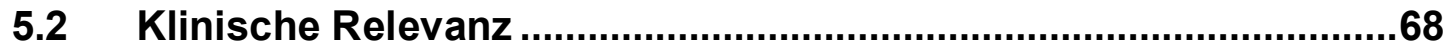

5.3 Kritische Beurteilung der Methoden .............................................71

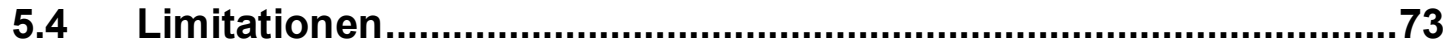




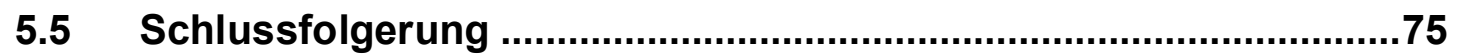

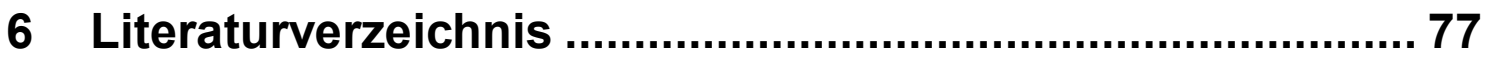

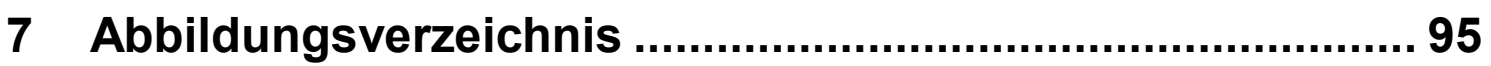

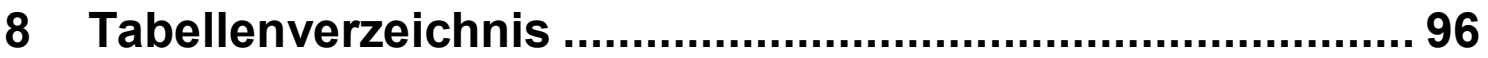

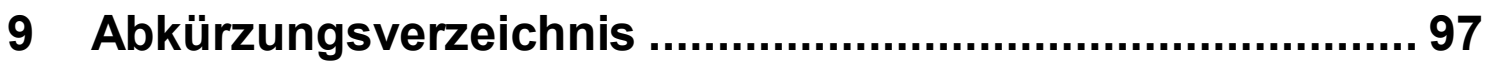

10 Curriculum Vitae.................................................................. 98

Schriftliche Erklärung .......................................................... 102 


\section{Zusammenfassung}

\subsection{Zusammenfassung auf Deutsch}

Zielsetzung: Das Ziel dieser Arbeit war die Beurteilung der diagnostischen Leistungsfähigkeit der virtuellen Noncalcium (VNCa) Dual-Energy-Computertomographie (DECT) für den Nachweis lumbaler Bandscheibenvorfälle im Vergleich zu der konventionellen Graustufen-Computertomographie. Dabei galt die Magnetresonanztomographie (MRT) als Referenzstandard.

Material und Methodik: Für diese retrospektive Studie wurden 41 Patienten (243 Bandscheiben; Durchschnittsalter 68 Jahre; 24 Frauen [Durchschnittsalter, 68 Jahre] und 17 Männer [Durchschnittsalter, 68 Jahre]) zwischen März 2017 und Januar 2018 einer klinisch indizierten DECT, durchgeführt mit einem DualSource-Computertomographie (DSCT)-Scanner der dritten Generation (Somatom Force; Siemens Healthineers, Forchheim, Deutschland) sowie einer 3.0Tesla MRT (Magnetom PrismaFit; Siemens Healthineers, Forchheim, Deutschland) im Abstand von maximal zwei Wochen unterzogen. Sechs Radiologen, verblindet hinsichtlich der klinischen und MRT-Informationen, untersuchten unabhängig voneinander die konventionellen Graustufen-DECT-Bildserien auf das Vorhandensein und den Grad eines Bandscheibenvorfalls sowie der Affektion der Spinalnervenwurzeln. Nach acht Wochen wurden die Bildserien von den gleichen sechs Untersuchern unter Verwendung von farbkodierten VNCa-Rekonstruktionen neu ausgewertet. Die MRT, die von zwei unabhängigen, erfahrenen und in Bezug auf klinische und DECT-Informationen verblindeten Radiologen ausgewertet wurde, diente dabei als Referenzstandard. Sensitivität und Spezifität waren die wichtigsten Kennzahlen der Diagnoseleistung.

Ergebnisse: Insgesamt wurden 112 Bandscheibenvorfälle der Lendenwirbelsäule in der MRT erfasst. VNCa zeigte insgesamt eine höhere Sensitivität (612 von 672 [91\%] versus (vs.) 534 von 672 [80\%]) und Spezifität (723 von 786 [92\%] vs. 665 von 786 [85\%]) zur Detektion eines lumbalen Bandscheibenvorfalls im Vergleich zur Standard-Computertomographie (CT) (alle Vergleiche, $P<0.001$ ). Die Interrater-Reliabilität war „exzellent“ für VNCa und "gut“ für die Standard-CT 
( $\mathrm{k}=0,82$ vs. 0,$67 ; P<0.001$ ). VNCa erreichte im Vergleich zum Standard-CT eine überlegene Diagnosesicherheit und Bildqualität bei niedrigerem Bildrauschen (alle Vergleiche, $P<0.001$ ).

Schlussfolgerung: Farbkodierte Dual-Energy CT VNCa-Rekonstruktionen zeigen im Vergleich zur Standard-CT eine signifikant bessere diagnostische Genauigkeit und Sicherheit für die Detektion eines lumbalen Bandscheibenvorfalls. 


\subsection{Abstract in English}

Purpose: The aim of this thesis was to evaluate the diagnostic performance of dual energy computed tomography (DECT) in combination with virtual non-calcium (VNCa) technique for the detection of lumbar disc herniations. Magnetic resonance tomography (MRT) is established as the gold standard. We assume that the DECT VNCa technique will ensure a similarly high sensitivity and specificity in the diagnostic process.

Materials and Methods: For this study, data sets of 41 patients (243 intervertebral disks; overall mean age, 68 years; 24 women [mean age, 68 years] and 17 men [mean age, 68 years]) were evaluated retrospectively. Between March 2017 and January 2018 , all of them underwent a clinically indicated and routinely performed third-generation DECT by a modern DSCT-scanner (Somatom Force; Siemens Healthineers, Forchheim, Germany) and a contrast-free 3.0 Tesla MRI (Magnetom PrismaFit; Siemens Healthineers, Forchheim, Germany). The interval between the two examinations was no longer than two weeks. Subsequently, six radiologists independently evaluated the conventional grayscale DECT image series. The question here was the presence and degree of a lumbar disc herniation and the affection of spinal nerve roots. All CT examiners were blinded with regarded to the clinical information and the results of the MRI. After eight weeks, the exams were re-evaluated by the testers using color-coded VNCa reconstructions. The MRI examinations, which were diagnosed by two independent, experienced radiologists blinded with regard to clinical and DECT information, served as a reference standard. Sensitivity and specificity were the key indicators of the study.

Results: A total of 112 herniated lumbar disks were depicted at MRI. VNCa showed higher overall sensitivity (612 of 672 [91\%] vs 534 of 672 [80\%]) and specificity (723 of 786 [92\%] vs 665 of 786 [85\%]) for detecting lumbar disk herniation compared with standard CT (all comparisons, $P<0.001$ ). Interreaderagreement was excellent for $\mathrm{VNCa}$ and good for standard CT ( $\mathrm{k}=0.82$ vs 0.67 ; 
$P<0.001)$. VNCa achieved superior diagnostic confidence, image quality, and noise scores compared to standard CT (all comparisons, $P<0.001$ ).

Conclusions: Compared to standard CT, color-coded dual-energy-CT virtual noncalcium reconstructions showed a significantly better diagnostic performance and confidence for the detection of a lumbar disc herniation. 


\section{Einführung}

\subsection{Hintergrund und Zielsetzung dieser Studie}

Der Bandscheibenvorfall im Bereich der Lendenwirbelsäule ist eine häufige degenerative Erkrankung des muskuloskelettalen (MSK) Systems. Als Folgen werden oftmals Schmerzen im unteren Rückenbereich beschrieben, welche mit erheblichen sozialen und wirtschaftlichen Belastungen verbunden sind. ${ }^{1-3}$

Komplikationen, wie die Kompression von Rückenmarksanteilen oder die Affektion von Spinalnervenwurzeln, können zu irreversiblen Schädigungen führen. ${ }^{4,5}$ Daher ist eine schnelle und akkurate Diagnostik des Bandscheibenvorfalls sowie möglicher Komplikationen von elementarer Bedeutung, um frühzeitig die optimale Therapie einleiten zu können und ein schlechtes Behandlungsergebnis zu vermeiden.

Derzeit repräsentiert die MRT den Goldstandard für die Visualisierung von Bandscheibenvorfällen, da sie eine hervorragende Abgrenzung zwischen Bandscheiben und Liquor ermöglicht. ${ }^{6}$ Im klinischen Alltag zeigen sich dennoch Einschränkungen, welche den Arbeitsablauf erschweren. So ist die Verfügbarkeit der MRT in der Regel an die regulären Arbeitszeiten gebunden und dadurch in der Benutzung limitiert. ${ }^{7}$ Hinzu kommen diverse absolute und relative Kontraindikationen, wie Cochlea-Implantate, verschiedene Herzschrittmacher, Klaustrophobie oder die häufig schmerzbedingte Unfähigkeit des Patienten still zu liegen. ${ }^{8}$ Darüber hinaus ist die MRT eine zeitaufwändige und kostenintensive Untersuchungsmethode ${ }^{7,8}$, weswegen Patienten mit starken Rückenschmerzen initial nicht selten einer CT anstelle einer MRT unterzogen werden. ${ }^{9}$

Eine Meta-Analyse aus dem Jahr 2012 zeigt eine mäßige Sensitivität $(77,4 \%)$ und Spezifität $(73,7 \%)$ der Standard-CT für die Detektion eines lumbalen Bandscheibenvorfalls. ${ }^{9}$ In einer jüngeren Untersuchung von Notohamiprodjo et al. $(2017)^{10}$ werden verbesserte Ergebnisse bei der Auswertung der diagnostischen Genauigkeit der herkömmlichen Single-Energy-Computertomographie (SECT) verglichen mit der MRT präsentiert. In dieser Studie wurden spezielle Rekonstruktionsalgorithmen angewandt, mithilfe derer für die diagnostische Genauigkeit eine Sensitivität pro Patient von $97,7 \%$ für die Detektion von Protrusionen 
erreicht werden konnte. Für den Nachweis einer Herniation liegt der Wert lediglich bei 70,9\%. Allerdings blieb der sichere Nachweis einer pathologischen Veränderung der Bandscheiben vor allem aufgrund des geringen Kontrasts zwischen Bandscheibe und Liquor weiterhin schwierig. ${ }^{10}$

Die DECT hat dank ihrer technischen Weiterentwicklung in Hinblick auf die Materialcharakterisierung neue Perspektiven in der Bildgebung eröffnet. ${ }^{711-14}$ Hierzu zählt beispielweise die Visualisierung von Knochenmarksödemen mit Hilfe von VNCa-Bildern in der muskuloskelettalen DECT ${ }^{15-21}$ Solche farbkodierten VNCa-Rekonstruktionen finden auch bei Arthrographien Anwendung. ${ }^{7,22}$ Zudem zeigte sich, dass Knochenmarksinfiltrationen bei Patienten mit Multiplem Myelom mittels DECT besser nachgewiesen werden können. ${ }^{23}$ Mit der Entwicklung der DSCT-Scanner der dritten Generation wurden vermehrt Algorithmen für VNCaRekonstruktionen in die Programme der Bildnachbearbeitung implementiert. Dabei wurde ein neuartiger VNCa-Nachbearbeitungsalgorithmus etabliert, welcher für die farbige Darstellung der lumbalen Bandscheiben zwei verschiedene Colour Lookup Table (CLUT) verwendet.

Diese Studie wurde anhand der Annahme konzipiert, dass farbkodierte VNCaBildserien, welche durch eine DECT mit einem modernen DSCT-System, die diagnostische Genauigkeit und Sicherheit für die Erkennung lumbaler Bandscheibenvorfälle bei kontrastmittelfreier DECT im Vergleich zu herkömmlicher Graustufen CT-Serien erheblich verbessern können. Grund für diese Annahme ist unter anderem die gesteigerte spektrale Trennung des verwendeten Scanners. ${ }^{24}$ Folglich war das Ziel dieser Doktorarbeit, die diagnostische Genauigkeit eines farbkodierten DECT VNCa-Rekonstruktionsalgorithmus für die Detektion eines lumbalen Bandscheibenvorfalls im Vergleich zu einer Standard-CT zu beurteilen. Dabei galt die MRT als Referenzstandard. 


\subsection{Epidemiologie}

In den westlichen Industrienationen repräsentieren degenerative Veränderungen der Lendenwirbelsäule und die damit einhergehenden Defizite eine der häufigsten Anlässe für eine Arztkonsultation. ${ }^{25-27}$ Im Gesundheitsbericht des Bundes für das Jahr 2015 werden die gesamten Krankheitskosten für Dorsopathien (10. Version International Classification of Diseases (ICD-10): M45-M54) mit 10,93 Milliarden Euro angegeben. ${ }^{28}$ Ein nicht unerheblicher Teil dessen entfällt dabei auf Diagnosen, die auf Erkrankungen beziehungsweise (bzw.) Veränderungen der lumbalen Bandscheiben zurückzuführen sind. ${ }^{1,27}$

Die absolute Fallzahl für die Diagnosegruppe "Sonstige Bandscheibenschäden“ (ICD-10: M51), in der zervikale und thorakale Bandscheibenschäden nicht mit inbegriffen sind, liegt für das Jahr 2017 in Deutschland bei 141.935. Davon waren 73.807 Patienten männlich und 68.128 Patienten weiblich.

Die altersspezifischen Fallzahlen je 100.000 Einwohner (Jahr 2017) sind wie folgt: ${ }^{29}$

- unter 15 Jahren: 0

- 15 bis unter 45 Jahren: 132

- 45 bis unter 65 Jahren: 254

- 65 Jahre und älter: 226

Daraus wird ersichtlich, dass der lumbale Bandscheibenvorfall einen Häufigkeitsgipfel im mittleren Lebensalter aufweist und im Kindesalter eine absolute Rarität darstellt.

Die Jahresprävalenz in Deutschland und anderen westlichen Industriestaaten, wie die Vereinigten Staaten von Amerika oder Frankreich, liegt zwischen 1\% $2,5 \%{ }^{27}$ Die Lebenszeitprävalenz bei 45 -bis 55 -jährigen liegt bei über $20 \% .{ }^{27}$

Weitaus am häufigsten von einem Bandscheibenvorfall betroffen sind dabei die beiden sich am kaudalen Ende befindenden Wirbelsäulensegmente L4/L5 und L5/S1. ${ }^{30-32}$ 


\subsection{Aufbau und Funktion der Bandscheiben}

Die Bandscheiben sind Strukturen, welche zwischen den Wirbelkörpern liegen und damit einen klar definierten Raum besetzen. ${ }^{33-35}$ Die Begrenzung dieses Raumes erfolgt kranial und kaudal durch die Bodenplatte bzw. Deckplatte des angrenzenden Wirbelkörpers. Außerdem verstärken ventral (Ligamentum Iongitudinale anterius) und dorsal (Ligamentum longitudinale posterius) Bandstrukturen den Intervertebralraum. ${ }^{35-37}$

Die Wirbelsäule des Menschen besteht in der Regel aus insgesamt 24 Wirbelkörpern. Darunter sind sieben Halswirbel (C1-C7), zwölf Brustwirbel (Th1-Th12) und fünf Lendenwirbel (L1-L5), an die sich kaudal das Kreuzbein (Os sacrum) anschließt, welches durch Verschmelzung der fünf Sakralwirbel (S1-S5) entsteht. Die Wirbel sind über 23 Bandscheiben miteinander verbunden. Die unterschiedliche Anzahl an Wirbelkörpern und Bandscheiben ergibt sich daraus, dass zwischen Atlas und Axis (erster und zweiter Halswirbel) regelhaft keine Bandscheibe vorhanden ist und das Os sacrum ein einziger Knochen ist. ${ }^{35}$ In unmittelbarer Nähe der Bandscheiben liegen posterolateral die Zwischenwirbellöcher (Foramina intervertebralia), die von den aus dem Rückenmark stammenden Spinalnerven durchzogen werden. ${ }^{35}$

Bei genauer Betrachtung bestehen die Bandscheiben im Wesentlichen aus drei Hauptkomponenten: Dem Anulus fibrosus (AF), dem innen liegenden Gallertkern (Nucleus pulposus, NP) und den, an den Oberflächen der Wirbelkörper liegenden, hyalinen Knorpeldeckplatten. ${ }^{33-35,38}$ Der AF besteht in seiner Außenzone aus konzentrisch angelagerten Lamellen straffen Bindegewebes, welche zum größten Teil aus Typ-I-Kollagen und einer geringen Zahl an elastischen Fasern aufgebaut sind. Die Innenzone des Faserrings ist hingegen aus faserknorpeligen Gewebe gebildet, welches in der Mehrheit aus Typ-I- und Typ-II-Kollagen besteht und ohne klar definierbare Grenze in das Gewebe des NP übergeht. ${ }^{33,34,39}$ Der Gallertkern ist, neben geringeren Anteilen an Kollagen-Typ-II und elastischen Fasern, reich an Aggrecan und anderen Proteoglykanen. An diese sind eine große Menge von Glykosaminoglykanen, wie Chondroitin-4-Sulfat, Chondroitin-6-Sulfat und Keratansulfat, gebunden, die aufgrund ihrer chemischen Eigenschaften in der Lage sind verhältnismäßig große Mengen an Wasser zu speichern. ${ }^{39,40}$ 
Dadurch stellt der NP eine Art „Wasserkissen“ dar. ${ }^{33-35,39}$ Die hyalinen Knorpeldeckplatten sind embryologisch betrachtet Teil der knorpelig angelegten Wirbelkörper, zählen aber anatomisch wie erwähnt zu der Bandscheibe. Bis in etwa zum fünften Lebensjahr sind diese und Anteile des $A F$ zunächst noch vaskularisiert. Im Erwachsenenalter obliterieren die versorgenden Gefäße zum größten Teil, weswegen die Bandscheiben dann nur noch über Diffusion ernährt werden. $^{33,34,39,41}$

Die Funktion der Bandscheiben besteht hauptsächlich darin, die durch Bewegung entstehenden Druck- und Schubkräfte auf die benachbarten Boden- und Deckplatten der angrenzenden Wirbelkörper sowie den AF zu übertragen und dadurch zu verteilen. ${ }^{33-35,42} 43$

Kommt es zu einer zentrischen Belastung auf die Zwischenwirbelscheibe wird der NP gleichmäßig in die Peripherie verlagert, wodurch die Kraft in Form von Zugbelastungen auf die Fasern des AF umgesetzt wird. Bei exzentrischer Belastung der Bandscheibe wird der NP in Richtung der entlasteten Seite verlagert und dadurch auf gleicher Seite die Fasern des AF unter Spannung gebracht. Dies hat den zusätzlichen Effekt, dass durch diesen Mechanismus auch der Bewegungsumfang um die Transversalachse (Ventralflexion und Dorsalextension), die Longitudinalachse (Rotation) sowie die Sagittalachse (Lateralflexion) begrenzt wird. $35,37,42,44$ 


\subsection{Pathologien der Bandscheiben}

Als Frühzeichen einer degenerativen Bandscheibenveränderung gelten Risse zwischen den Fasern des AF. Im Frühstadium kommt es dabei nicht zu Verlagerungen von Anteilen der Bandscheibe über den Bandscheibenraum hinaus. Diese Risse können sich in allen drei Hauptebenen ausbreiten und werden demnach eingeteilt in radiär, konzentrisch und transversal. ${ }^{36,45}$

Im Gegensatz dazu definiert die Herniation, bezogen auf die Bandscheibenpathologien, ganz allgemein die Verlagerung von Bandscheibenmaterial über die anatomische Begrenzung des Bandscheibenraums hinaus. Das beschriebene Gewebe kann dabei Anteile des NP, des AF oder der Ringapophyse enthalten. ${ }^{36,46}$

Nach der aktuellen Nomenklatur ist die Herniation lediglich ein Überbegriff. Man unterscheidet demnach die breitbasige Herniation und die fokale Herniation wobei die Unterscheidung auf mathematischen Gesichtspunkten basiert. So liegt eine breitbasige Herniation vor, sobald mehr als $25 \%$ bis maximal $50 \%$ des Bandscheibenumfangs betroffen sind. Bei weniger als $25 \%$ spricht man hingegen von einer fokalen Herniation, die wiederum in Protrusion und Extrusion unterteilt werden kann. ${ }^{45}$ Dabei gilt:

- Protrusion: Die Distanz an der Basis der Vorwölbung ist größer als die Distanz an den Endpunkten (Abbildung (Abb.) 1, A). ${ }^{46}$

- Extrusion: Die Distanz an der Basis der Vorwölbung ist geringer als die Distanz an den Endpunkten (Abb. 1, B). ${ }^{46}$

Eine Sonderform der Extrusion ist die Sequestration, bei der ein Teil in den Epiduralraum disloziert und die Verbindung zu der dazugehörigen Bandscheibe verliert (Abb. 1, C) ${ }^{46}$

Der Großteil der Herniationen erfolgt dabei nach mediolateral in Richtung der Foramina intervertebralia, lateral des Ligamentum longitudinale posterius. Mediale Vorfälle, die mit einer Zerreißung des Ligaments einhergehen, laterale oder ventrale Herniationen sind vergleichsweise seltener zu beobachten. ${ }^{43}$ 


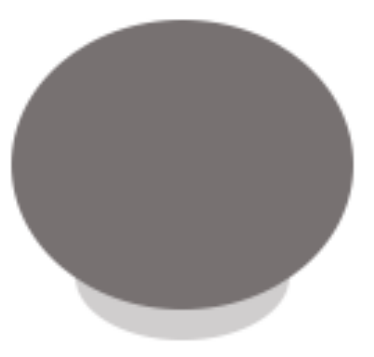

A

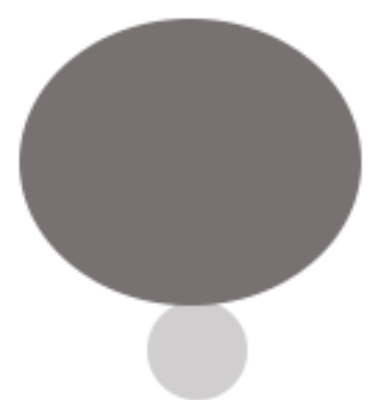

B

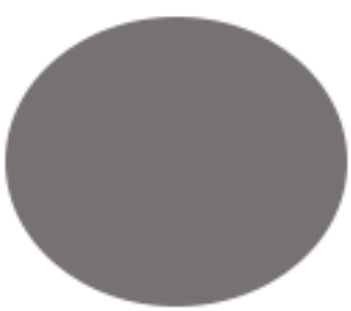

C

Abbildung 1: Formen der fokalen Herniation; modifiziert nach Konieczny et al., $2020^{47}$

A: Schematische Darstellung einer Protrusion.

B: Schematische Darstellung einer Extrusion.

C: Schematische Darstellung einer Sequestration.

Der dislozierte Anteil der Bandscheibe (hellgrau) ist jeweils heller abgebildet. Die Darstellungen demonstrieren eine axiale Sicht.

Sind mehr als $50 \%$ des Bandscheibenumfangs von einer Vorwölbung betroffen spricht man von „Bulging“. In dieser Kategorie unterscheidet man weiter zwischen einem symmetrischen Bulging (Abb. 2, A), bei dem die gesamte Zirkumferenz betroffen ist, und einem asymmetrischen Bulging (Abb. 2, B), bei dem mehr als $50 \%$, jedoch nicht die gesamte Bandscheibe eine Herniation aufweist. Das Bulging ist allerdings nach aktueller Definition keine pathologische Bandscheibenveränderung. ${ }^{32,45,47}$
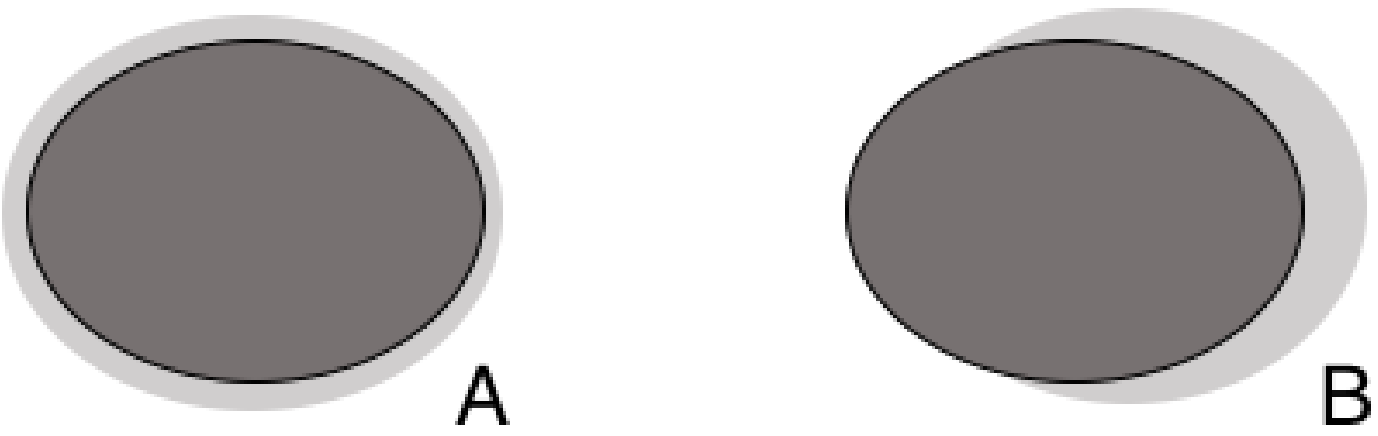

Abbildung 2: Bulging von Bandscheiben; modifiziert nach Fardon et al., $2001^{36}$
A: Symmetrisches Bulging
B: Asymmetrisches Bulging 


\subsection{Pathogenese}

Die Entstehung von Bandscheibenvorfällen ist ein komplexes, multifaktorielles Geschehen, bei dem insbesondere Alterungsprozesse im Vordergrund stehen. ${ }^{33,34,48-50}$ Prinzipiell muss man in diesem Punkt zwischen der physiologischen Alterung und der degenerativen Bandscheibenerkrankung, die häufig mit chronischen Rückenschmerzen assoziiert ist, unterscheiden. Der Übergang dabei ist jedoch fließend. Die degenerative Bandscheibenerkrankung beschreibt dabei eine vorzeitige und beschleunigte Alterung.

Nachweisbare biochemische Veränderungen beginnen schon in sehr frühen Lebensabschnitten. ${ }^{51}$ Neben dem Verlust an Proteoglykanen - vor allem Aggrecan - der schon im Kindesalter beginnt, ändert sich im NP auch das Verhältnis von Keratansulfat zu Chondroitin-4- und Chondroitin-6-Sulfat zugunsten des Keratansulfats. Das führt dazu, dass aufgrund der geringeren Menge an Proteoglykanen und des verminderten hydrophilen Potenzials des Keratansulfats weniger Wassermoleküle gebunden werden können. Korrespondierend dazu kommt es sowohl im NP als auch im AF zu einer Zunahme und Umstrukturierung von Kollagenfasern. ${ }^{52}$ Dies hat unter anderem zur Folge, dass es mit zunehmenden Alter zu einer messbaren Höhenminderung der Bandscheibe kommt. ${ }^{42,53}$ Gleichzeitig sinkt dadurch aber auch der Expansionsdruck des NP, was zum Teil begründet, warum der lumbale Bandscheibenvorfall seinen Häufigkeitsgipfel im mittleren Lebensalter hat und die Inzidenz anschließend wieder abnimmt. ${ }^{27}$

Die Bandscheiben sind zudem das größte nichtvaskularisierte Gewebe im menschlichen Körper, was für die Pathogenese von Bandscheibenherniationen ein weiterer wichtiger Aspekt ist. ${ }^{22}$ Die Ernährung erfolgt über Diffusion aus zwei an die knorpeligen Endplatten angelagerten kapillaren Plexus, wobei Anteile des NP mehrere Millimeter von dieser Nährstoffquelle entfernt liegen können. ${ }^{54}$ Daraus resultiert eine teilweise anaerobe Stoffwechsellage der im NP ubiquitär enthaltenen Chondrozyten, deren Funktion die Produktion von Proteoglykanen und Kollagenfasern ist. Die Mikroumgebung kann dabei höhere Konzentrationen an Laktat und dadurch einen niedrigeren $\mathrm{pH}-\mathrm{Wert}$ aufweisen, was wiederum einen negativen Effekt auf die Zellfunktion hat und begünstigt, dass die Zelldichte vor allem im NP mit steigendem Alter deutlich abnimmt. ${ }^{55}$ 
Ein weiterer wichtiger Punkt für den Degenerationsprozess sind genetische Einflussfaktoren. So konnte in Zwillingsstudien gezeigt werden, dass es eine Prädisposition für eine degenerative Bandscheibenerkrankung gibt. ${ }^{56}$ Zudem existieren eine Vielzahl von genetischen Polymorphismen, die relevante Stoffwechselwege der Bandscheiben beeinflussen und dadurch das sensible Gleichgewicht zwischen anabolen und katabolen Vorgängen stören können. ${ }^{34,49,57}$ Es gibt eine Reihe von Genen, für die nachgewiesen werden konnte, dass genetische Veränderungen das Risiko für Bandscheibenvorfälle erhöhen kann. ${ }^{58}$ Martirosyan et al. $(2016)^{58}$ beschreiben dabei Gene die für Matrix-Metalloproteinasen, Apoptosefaktoren und Wachstumsfaktoren kodieren.

Alle diese Vorgänge münden, insbesondere durch eine Verringerung des Wasseranteils im NP in einer erhöhten Steifigkeit der Bandscheibe ${ }^{59,60}$ Durch die reduzierte Flexibilität können exogen einwirkende Kräfte nicht mehr adäquat, entsprechend der eigentlichen Bandscheibenfunktion, auf die verschiedenen Komponenten der Zwischenwirbelscheibe umgesetzt werden. Auf diese Weise bilden sich kleine Risse im AF, die aufgrund geringer Heilungstendenz als Frühzeichen für eine Bandscheibendegeneration gelten. ${ }^{32,45}$

Der Bandscheibenvorfall entsteht letztendlich durch ein Zusammenspiel aus endogenen Faktoren und weiteren exogenen Einflüssen. ${ }^{27}$ Als Risikofaktoren sind an dieser Stelle vor allem Adipositas, mangelnde Bewegung, Rauchen, ein gesteigerter Alkoholkonsum und Fehlstellungen der Wirbelsäule, wie eine Skoliose oder eine Hyperlordose zu nennen. ${ }^{27,61,62}$ Eine zentrale Rolle spielt zudem auch die durch die individuelle Lebensweise entstehende, biomechanische (Fehl)Belastung der Bandscheiben. ${ }^{50,61-64}$ Kritisch sind insbesondere Bewegungsmuster, die eine vermehrte anteriore Kompression der Bandscheibe bewirken, wie zum Beispiel das Anheben eines schweren Gegenstandes mit nach vorne gebeugtem Oberkörper. Bei dieser Kombination entsteht ein maximaler Druck auf der Bandscheibe. ${ }^{27}$ Sind nun, wie zuvor beschrieben, bereits Mikrotraumata in Form anulärer Risse vorhanden, können sich Anteile des NP dorthin verlagern und gegebenenfalls eine Bandscheibenherniation provozieren.

Neben der Degeneration können auch Infektionen im Rahmen einer Spondylodiszitis oder andere entzündliche Erkrankungen als Prädiktoren für einen Bandscheibenvorfall angesehen werden. ${ }^{65,66}$ 
Degenerativ bedingte Herniationen stehen den akut-traumatischen Herniationen gegenüber, welche durch Extrembelastungen der Bandscheiben entstehen. ${ }^{27}$ Mögliche Auslöser können hierbei Verkehrsunfälle oder die Durchführung von Hochleistungssportarten wie Turnen sein. Dabei kommt es in der Regel parallel zu einem Riss im AF zu einer direkten Verlagerung von Gewebe des NP. Häufig entstehen dabei zusätzlich knöcherne sowie ligamentäre instabile Begleitverletzungen. ${ }^{27,67}$ Der Anteil an traumatisch bedingten Bandscheibenvorfällen ist jedoch allgemein wesentlich geringer als der der degenerativ bedingten Herniationen. ${ }^{27,68,69}$ In den Studien von Apple et al. $(1987)^{68}$ und Terhaag und Frowein (1989) ${ }^{69}$ lag die Inzidenz traumatischer Herniationen bei circa 1\%. 


\subsection{Klinische Symptomatik und Untersuchung}

Bevor es zur eigentlichen Herniation kommt, berichten Patienten häufig von bereits länger bestehenden oder intermittierenden Rückenschmerzen, welche in den meisten Fällen als Hinweise auf degenerative Prozesse gedeutet werden können. ${ }^{27}$ Der lumbale Bandscheibenvorfall als Akutereignis ist klassischerweise charakterisiert durch plötzlich einsetzende Schmerzen im unteren Rückenbereich (Lumbalgie). Durch die Affektion nervaler Strukturen wie Spinalnervenwurzeln wird dieser in den meisten Fällen von ausstrahlenden Schmerzen in das Bein der betroffenen Seite und Kribbelparästhesien im Ausbreitungsgebiet der beteiligten Nervenwurzel begleitet. ${ }^{27,30}$ Ist eine ventrale Schmerzausstrahlung (Femoralgie) vorhanden, deutet dies auf eine Schädigung in Höhe L4 oder weiter kranial abgehender Nervenwurzeln hin. Bei dorsaler Schmerzausbreitung (Ischialgie) liegt die Ursache meist im Bereich der L5- oder S1-Wurzel. Je nach Ausmaß der Herniation können außerdem dermatombezogene Sensibilitätsstörungen, Paresen der Kennmuskeln und Reflexausfälle auftreten. ${ }^{30,70}$ Durch eine Rückenmarkskompression kann es dabei auch zu neurologischen Funktionsausfällen von tiefer austretenden Nervenwurzeln kommen. Ein - oftmals einseitiger paravertebraler Hartspann sowie eine Druckdolenz sind weitere häufige Symptome. $^{27}$

Als spezielle Verlaufsformen eines lumbalen Bandscheibenvorfalls sind das Conus medullaris-Syndrom (CMS), das Cauda equina-Syndrom (CES), die Kombination beider Krankheitsbilder, welche als Conus-Cauda-Syndrom bezeichnet wird und das kompressionsbedingte Absterben der Spinalnervenwurzel („Wurzeltod“) zu nennen. ${ }^{30}$

CMS und CES sowie die kombinierte Form sind meist die Folge von ausgeprägten raumfordernden Prozessen, die eine massive Kompression des Rückenmarks und der Spinalnerven bewirken. Das CMS tritt dabei infolge einer Herniation auf Höhe Th12 - L2 auf, da dort regelhaft das kaudale Ende des Myelons liegt. Je nach Größe des Vorfalls zeigt sich klinisch ein isoliertes CMS oder, bei zusätzlicher Einengung der Fasern der Cauda Equina, ein kombiniertes ConusCauda-Syndrom. ${ }^{71}$ Beim CMS sind vor allem die Segmente S3 - S5 betroffen. 
Daher ist es charakterisiert durch eine Störung der Blasen- und Mastdarmfunktion, resultierend in einer Überlaufblase und dem Verlust des analen Sphinktertonus, einer Reithosenanästhesie, Impotenz und abgeschwächtem oder fehlendem Analreflex. ${ }^{72}$ Ein isoliertes CES ist meist die Folge großer posteromedialer Bandscheibenvorfälle auf Höhe L3 - L4. In dieser Position werden ausschließlich die Fasern der Cauda equina komprimiert. ${ }^{73}$ Das klinische Bild ist meist asymmetrisch und beinbezogen und präsentiert sich mit schlaffen Paresen, Sensibilitätsstörungen und abgeschwächten oder fehlenden Muskeleigenreflexen der unteren Extremität. ${ }^{30,73}$ Beim Conus-Cauda-Syndom zeigen sich die zuvor beschriebenen Symptomkomplexe kombiniert. ${ }^{73}$

Ein drohender Wurzeltod kündigt sich klassischerweise durch eine Abnahme der Schmerzsymptomatik bei gleichzeitiger Zunahme der Paresen der betroffenen Kennmuskeln an. Alle beschriebenen Sonderformen sind absolute Notfallindikationen und bedürfen einer umgehenden operativen Therapie. 1,4,5,27,70,74,75 Im diagnostischen Prozess ist der wichtigste Aspekt daher eine ausführliche und präzise Anamnese. Dabei gilt es zunächst die sogenannten „red flags“ (Tabelle 1) beim Auftreten von Rückenschmerzen zu evaluieren. Diese bewerten neben den neurologischen Defiziten auch die Frage nach weiteren gefährlichen Grunderkrankungen wie Frakturen, Tumore oder Infektionen. ${ }^{70}$ 


\section{Mögliche Ätiologie Klinische und anamnestische Hinweise}

\begin{tabular}{|c|c|}
\hline Fraktur & $\begin{array}{l}\text { - relevantes Trauma (im Verhältnis zum Alter) } \\
\text { - längere systemische Steroidtherapie } \\
\text { - Bagatelltraum bei bekannter oder Verdacht auf Osteoporose }\end{array}$ \\
\hline Tumor & $\begin{array}{l}\text { - bekanntes Tumorleiden } \\
\text { - B-Symptomatik (ungewollter Gewichtsverlust, nächtliches } \\
\text { Schwitzen, Fieber) }\end{array}$ \\
\hline Infektion & $\begin{array}{l}\text { - vorangegangene Infiltrationsbehandlung der Wirbelsäule } \\
\text { - intravenöser Drogenabusus } \\
\text { - Immunsupression } \\
\text { - B-Symptomatik }\end{array}$ \\
\hline Radikulopathie & $\begin{array}{l}\text { - Symptome eines CMS/CES/Conus-Cauda-Syndrom (siehe Kapi- } \\
\text { tel 2.6) } \\
\text { - Symptome eines drohenden Wurzeltodes (siehe Kapitel 2.6) }\end{array}$ \\
\hline
\end{tabular}

Tabelle 1: "Red flags" bei akuten lumbalen Rückenschmerzen, modifiziert nach Will et al. (2018) ${ }^{76}$ und Casser et al. $(2016)^{70}$

Von Bedeutung bei der Anamneseerhebung ist außerdem eine gezielte Familienanamnese, der Beginn der Symptomatik mit eventueller Vorgeschichte, die Schmerzlokalisation, die Art und Qualität der Schmerzen und die Frage nach bereits erfolgten Therapiemaßnahmen. ${ }^{27}$

In der sich anschließenden klinischen Untersuchung wird zunächst die Wirbelsäule inspiziert in Hinblick auf eine eventuelle Schonhaltung bzw. Form- oder Verlaufsanomalien wie eine bestehende Skoliose oder eine lumbale Hyperlordose. Patienten zeigen dabei häufig eine Lateralflexion des Rumpfes zur Entlastung des Beins der betroffenen Seite. Bei der Palpation muss vor allem auf einen muskulären Hartspann und die Schmerzprojektion geachtet werden. Die neurologische Untersuchung beinhaltet die Prüfung der Sensibilität, der Kraft der Kennmuskeln, der Muskeleigenreflexe und der Wurzeldehnungszeichen. ${ }^{27,64,70,75,77,78}$ Letztere können durch verschiedene Tests geprüft werden, wobei die diagnostische Aussagekraft der einzelnen Tests gering ist. ${ }^{75,79} \mathrm{Im}$ klinischen Alltag gängig ist unter anderem der Lasègue-Test, bei dem der Patient auf dem Rücken liegt 
und es durch das Anheben des durchgestreckten Beines der betroffenen Seite zu einschießenden Schmerzen im Versorgungsgebiet der beeinträchtigten Nervenwurzel kommt. Dies gilt als Hinweis auf eine Wurzelreizung im Bereich L4 S1. Der sogenannte umgekehrte Lasègue-Test verläuft äquivalent mit dem Unterschied, dass sich der Patient in Bauchlage befindet. So generierte Schmerzen lassen auf eine Wurzelschädigung Höhe L3 - L4 schließen. ${ }^{75,78,79}$ 


\subsection{Therapie}

Ziel der Therapie bei lumbalen Bandscheibenvorfällen ist die Rückbildung der Symptomatik und die soziale Reintegration. ${ }^{27}$ Wichtig ist, dass die Wahl des Therapiekonzeptes primär auf der Beschwerdesymptomatik des Patienten basiert und nicht auf der radiologischen Bildgebung, da beide Modalitäten nicht zwangsläufig miteinander korrelieren. In Studien konnte gezeigt werden, dass Bandscheibenherniationen bei Patienten mit steigendem Alter in der MRT häufiger diagnostiziert werden können, aufgrund der Beschwerdefreiheit allerdings keinerlei therapeutische Konsequenz besitzen. ${ }^{80,81}$

Die Therapie der ersten Wahl ist ein multimodales, konservatives Vorgehen, bestehend aus der Aufklärung des Patienten, einer frühzeitigen Pharmakotherapie, spezieller Bewegungstherapie sowie physikalischer und manueller Therapie. ${ }^{6,82-}$

${ }^{89}$ Es muss darüber aufgeklärt werden, dass eine Entlastung und Ruhigstellung in der Akutphase nicht förderlich sind. Der Patient sollte daher, bei erhaltener Mobilität und unabhängig von einer etwaigen radikulären Symptomatik, dazu aufgefordert werden weiter seinen normalen Aktivitäten nachzugehen und eine Bettruhe zu vermeiden. ${ }^{70,90-92}$

Eine adäquate Schmerztherapie sollte unterstützend angewendet werden. Angelehnt an das Stufenschema der Weltgesundheitsorganisation zur Behandlung von Tumorschmerzen werden initial Nicht-Opioid-Analgetika, insbesondere Nicht-steroidale Antirheumatika (NSAR) verabreicht. Bei Kontraindikationen für NSAR oder bei fehlender Wirkung können stattdessen selektive Cyclooxygenase-2-Hemmer wie Celecoxib genutzt werden. Bei weiterhin insuffizienter Analgesie kann außerdem die Gabe niedrigpotenter Opioide, wie Tilidin oder Tramadol über zwei bis drei Wochen unter engmaschiger Kontrolle in Erwägung gezogen werden. ${ }^{70,93}$ Muskelrelaxantien können bei begleitenden Verspannungen kurzfristig additiv gegeben werden, wobei eine entsprechende Nutzen-Risikoeinschätzung aufgrund potenzieller Suchtgefahr erfolgen sollte. ${ }^{30}$ Bei einer Chronifizierung des Schmerzes können trizyklische Antidepressiva wie Gabapentin als Ko-Analgetika einen positiven Effekt haben, wobei die Evidenz für deren Verwendung gering ist. ${ }^{94}$ Wärmebehandlungen und zielgerichtete physiotherapeutische Anwendungen komplettieren das bevorzugte Therapiekonzept. 
In der Mehrzahl der Fälle bessert sich durch die konservative Therapie die Beschwerdesymptomatik in einem linearen Verlauf innerhalb von zwei bis vier Wochen. ${ }^{27,77}$ Sollte es trotz der eingeleiteten Maßnahmen zu einer Schmerzpersistenz oder Verschlechterung der Beschwerden kommen, kann als nächster Schritt eine periradikuläre Therapie mit einem Lokalanästhetikum und/oder einem Steroid durchgeführt werden. ${ }^{70,77}$ Dabei wird in der Regel unter Durchleuchtung oder computertomographisch gesteuert eine Nadel nahe der betroffenen Nervenwurzel positioniert und das Medikament injiziert. Diese minimal-invasive Anwendung hat eine therapeutische Intention, kann bei unklaren Schmerzsyndromen aber auch als diagnostisches Instrument angesehen werden. ${ }^{70}$

Als letztes Mittel bei länger als sechs Wochen bestehender klinischer Symptomatik und korrespondierenden radiologischen Befunden kann ein operatives Vorgehen in Erwägung gezogen werden.

Unabhängige Notfallindikationen für eine Operation sind das CMS, das CES, die Kombination der beiden Syndrome, das Conus-Cauda-Syndrom, und der drohende Wurzeltod, der mit progredienten oder akut auftretenden schweren motorischen Ausfällen einhergeht. ${ }^{30,34,77,95}$

Ziele der Operation sind die sichere und definitive Entlastung der betroffenen Spinalnerven bzw. des Conus medullaris oder der Cauda equina, ein möglichst geringes Weichteiltrauma durch den operativen Zugang, der Stabilitätserhalt des Bewegungssegments und nach Möglichkeit der Erhalt der Bandscheibe. ${ }^{27}$ Als Operationsverfahren werden je nach Morphologie der Herniation verschiedene Methoden angewandt. Als Standardverfahren gilt zurzeit die offene, mikrochirurgische Sequestrektomie, wobei je nach Befund auch perkutane Verfahren wie die Chemonukleolyse oder die perkutane automatisierte Diskotomie und Laserdiskotomie zum Einsatz kommen. ${ }^{27,30,95}$

Im Langzeitverlauf konnte beobachtet werden, dass hinsichtlich Beschwerdesymptomatik und Behinderungsgrad weder konservative noch operative Therapiekonzepte eine entsprechende Signifikanz aufweisen. ${ }^{96}$ Allerdings bilden sich bei den frühzeitig operativ versorgten Patienten die Schmerzen und die neurologischen Defizite schneller wieder zurück. ${ }^{97,98}$ Liegt jedoch ein chronifizierter Rückenschmerz ohne radikuläre Komponente vor, so bessert sich die Symptomatik meist nicht durch eine operative Intervention. ${ }^{99,100}$ 


\subsection{Bildgebende Verfahren}

Nach aktuellen Empfehlungen sollte bei Patienten mit chronischen, unspezifischen Rückenschmerzen innerhalb von vier Wochen keine standardmäßige Bildgebung erfolgen, weswegen die Indikation erst nach einer genauen und präzisen körperlichen Untersuchung gestellt wird. ${ }^{6}$ Als Ausnahmen gelten dabei Patienten, die Zeichen für einen spezifischen Rückenschmerz mit entsprechender Symptomatik aufweisen (,red flags“). ${ }^{70}$ Zur Diagnosesicherung eines lumbalen Bandscheibenvorfalls ist bei begründetem Verdacht eine bildgebende Untersuchung unerlässlich. ${ }^{27}$

Das Röntgen wird bei Patienten mit unteren Rückenschmerzen aufgrund der schnellen Verfügbarkeit, der niedrigen Strahlenbelastung und geringer Kosten meist initial durchgeführt und dient vor allem dem Ausschluss von frischen Frakturen als Auslöser für eine Lumbalgie. Aufgrund der fehlenden Möglichkeit zur direkten Darstellung von Weichteilgeweben und der damit verbundenen geringen Sensitivität spielt das Röntgen eine eher untergeordnete Rolle für die Diagnostik einer lumbalen Bandscheibenherniation. ${ }^{6}$ Dennoch können eine kompensatorische Skoliose, ein verschmälerter Zwischenwirbelraum oder osteophytäre Anbauten indirekte Hinweise sein und sollten bei entsprechenden Befunden in der körperlichen Untersuchung Anlass für eine weiterführende Diagnostik sein. ${ }^{6,66}$ Insbesondere osteophytäre Anbauten an den Wirbelkörpern lassen auf eine fortgeschrittene Degeneration des MSK Systems inklusive der Bandscheiben schlieBen, wodurch das Risiko einen Bandscheibenvorfall zu erleiden erhöht sein kann. ${ }^{63}$

Bei Verdacht auf einen lumbalen Bandscheibenvorfall sollte eine Schnittbildgebung erfolgen. Aktuell gilt die MRT als Goldstandard für den Nachweis eines lumbalen Bandscheibenvorfalls. $6,66,77,78,101,102$ Das Prinzip der MRT ist komplex. Es wird genutzt, dass Wasserstoffprotonen positiv geladene Teilchen des Atomkerns sind und einen Eigendrehimpuls (Spin) besitzen. Daher erzeugen sie ein messbares, elektromagnetisches Feld. ${ }^{103}$ Durch das Anlegen eines starken Magnetfeldes werden die Wasserstoffprotonen entsprechend der Feldlinien (ZAchse, Abb. 3) ausgerichtet, was man als Längsmagnetisierung bezeichnet. Im anschließenden Schritt wird ein hochfrequenter Impuls, die sogenannte Larmor- 
frequenz, in das Magnetfeld eingestrahlt, wodurch die Protonen synchronisiert, beziehungsweise angeregt werden. ${ }^{102,103}$ Durch diesen Vorgang kreist die Magnetisierung in der XY-Ebene, was als transversale Magnetisierung verstanden wird. In der Empfangsspule wird nun ein MR-Signal messbar. In Folge dessen bewirken die T1-Relaxation und die T2-Relaxation, dass diese transversale Magnetisierung abnimmt und der Ausgangszustand wieder erreicht wird. Beide Vorgänge laufen dabei parallel aber vollkommen unabhängig voneinander ab. ${ }^{102} \mathrm{Die}$ T1-Relaxation ist die longitudinale Relaxation und beschreibt den Vorgang, dass sich die Protonen nach Abschalten des Impulses wieder entlang des Magnetfeldes (Z-Achse) ausrichten. Die Zeitkonstante T1 ist unter anderem abhängig von der Stärke des Magnetfeldes. Die T2-Relaxation ist in diesem Fall die transversale Relaxation und beschreibt den Vorgang der Dephasierung der Spins, also den Verlust der Phasengleichheit. T1- und T2-Zeit sind spezifisch für die unterschiedlichen Gewebetypen. Um einen Bildkontrast zu erhalten werden die Protonen mehrmals nacheinander angeregt und die T1- und T2-Zeiten bestimmt. Die Zeit zwischen den einzelnen Anregungen wird als Repetitionszeit bezeichnet und beeinflusst vor allem den T1-Kontrast. Bei kurzer Repetitionszeit (<600 ms) richten sich weniger Protonen wieder in Längsrichtung aus und erzeugen dadurch bei einer erneuten Anregung ein geringeres Signal. Gewebe mit kurzem T1 (z.B. Fett) geben daher ein hohes Signal ab und erscheinen dadurch in T1-gewichteten Aufnahmesequenzen hyperintens. Wasser hingegen hat ein eher langes T1 und erscheint hypointens. Je nach Wichtung können so unterschiedliche Kontraste erzeugt und später im eigentlichen Schnittbild sichtbar gemacht werden. ${ }^{102-104}$ 

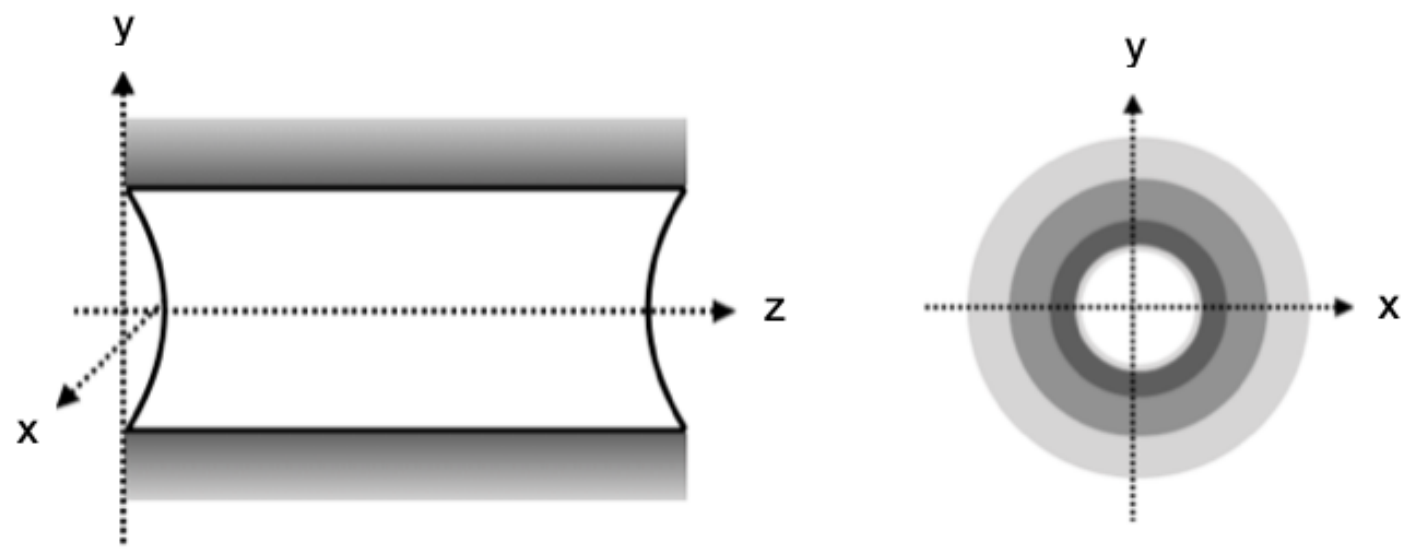

Abbildung 3: Achsenbezeichnung bei der MRT

Veranschaulicht ist die Achsenbezeichnung bei der MRT. Die Z-Achse verläuft dabei in Längsrichtung. Die XY-Ebene wird als Transversalebene bezeichnet.

(Abbildung modifiziert nach: Pabst (2013), Magnetresonanz-Tomographie, Lernskript für Mediziner $)^{104}$

Bei der Diagnose eines lumbalen Bandscheibenvorfalls mittels MRT ergeben sich einige Vorteile. Ein entscheidender Punkt ist die fehlende Exposition gegenüber ionischer Strahlung während des Untersuchungsvorgangs. Der visuelle Vorteil der MRT gegenüber anderen bildgebenden Verfahren ergibt sich vor allem durch den sehr guten Weichteilkontrast, der eine deutlich bessere Unterscheidung der einzelnen Bestandteile der Bandscheibe ermöglicht und dadurch auch die Darstellung der angrenzenden Bandstrukturen zulässt. ${ }^{6}$ Auch gegenüber dem Liquor ist die Bandscheibe mittels MRT sehr gut abgrenzbar, was in Summe dazu führt, dass die MRT die derzeit bevorzugte Bildgebung darstellt. ${ }^{6,10}$

Eine alternative Untersuchungstechnik ist die CT. Heutzutage werden standardmäßig sogenannte Multi-Detektor-Computertomographen (MDCT) verwendet, die erstmals 1998 eingeführt wurden. ${ }^{105} \mathrm{Im}$ Gegensatz zu den früher gängigen Geräten, die mit nur einem Detektorenkranz ausgestattet waren, verfügen diese Mehrschicht-Computertomographen mittlerweile über bis zu 256 Zeilen. Der Vorteil, der sich daraus ergibt, ist vor allem die signifikant kürzere Untersuchungsdauer, da ein wesentlich größerer Volumenbereich in der gleichen Zeit abgedeckt werden kann. Hinzu kommt, dass die Auflösung durch die Wahl dünnerer kollimierter Schichten deutlich verbessert werden konnte. Die kollimierte Schichtdi- 
cke beschreibt dabei die Breite des von der Röntgenröhre ausgehenden Strahlenfächers in Patientenlängsrichtung. Diese enormen technischen Fortschritte sind auch in der Diagnostik von Bandscheibenvorfällen relevant. So konnten Notohamiprodjo et al. $(2017)^{10}$ zeigen, dass mithilfe der MDCT eine lumbale Bandscheibenherniation durchaus sicher nachgewiesen werden kann und als Alternative zur MRT anzusehen ist. Bezogen auf die diagnostische Genauigkeit pro Patient lag die Sensitivität für die Detektionen einer Protrusion bei $97,7 \%$ und die Spezifität bei 99,0\%. Für den Nachweis einer Herniation konnte jedoch nur eine Sensitivität von 70,9\% erzielt werden (Spezifität: 99\%). Das Hauptproblem war allem der geringe Kontrast zwischen Liquor und Bandscheibenmaterial. ${ }^{10}$

Die CT ist deutlich weniger kostenintensiv und in der Regel schneller verfügbar als die MRT. 9,106 Durch die deutlich kürzere Untersuchungsdauer ist sie zudem weniger anfällig für Bewegungsartefakte und dadurch vorteilhaft bei Patienten, die nicht kooperativ sind oder schmerzbedingt nicht lange ruhig liegen können. ${ }^{106}$ Auch aufgrund der oben genannten Gründe wird im klinischen Alltag bei Patienten mit einer Lumbalgie und dem begründetem Verdacht auf einen potenziell interventionsbedürftigen Auslöser initial oftmals eine CT anstelle einer MRT durchgeführt. $^{9}$

Eine weitere diagnostische Möglichkeit, die vor der Entwicklung der CT und MRT häufiger zum Einsatz kam, ist die Myelographie. Deren Prinzip besteht darin, Kontrastmittel in den Subarachnoidalraum, also intrathekal, zu verabreichen und anschließend mittels Röntgenstrahlung (heutzutage vor allem mittels CT) Bilddatensätze zu akquirieren. Es konnte gezeigt werden, dass die diagnostische Genauigkeit für den Nachweis einer lumbalen Bandscheibenherniation vergleichbar ist mit der der MRT. ${ }^{107}$ Allerdings wird die Myelographie aufgrund ihrer Invasivität heutzutage meist nur noch zum Nachweis bestimmter Krankheitsbilder wie einer Spinalkanalstenose oder einer Liquorleckage verwendet. ${ }^{108}$

Weitere bildgebende Untersuchungen wie die Diskographie oder die PositronenEmissions-Tomographie sind Techniken, die potenziell den Nachweis eines lumbalen Bandscheibenvorfalls erlauben, allerdings in der routinemäßigen Diagnostik keine Rolle spielen. 


\subsection{Dual-Energy-Computertomographie}

Die Technik, auf welcher die DECT basiert, wurde bereits vor über 30 Jahren entwickelt. ${ }^{109}$ Prinzipiell beschreibt die DECT die Aufnahme zweier CT-Scans bei unterschiedlichen Energieniveaus. Der klinische Nutzen wurde in den Anfangszeiten durch die Untersuchungsdauer und die damit verbundene schlechte zeitliche Auflösung erheblich beeinträchtigt, da beide Aufnahmen in kurzer Abfolge aber nicht zeitgleich erfolgten. ${ }^{7}$

So wurden erst Mitte der 2000er die ersten Dual-Source-Computertomographen auf den Markt gebracht, die in einem effizienten Dual-Energy-Modus betrieben werden konnten und in den klinischen Alltag implementiert wurden. ${ }^{110}$ Heutzutage gibt es vier unterschiedliche Techniken, welche die Aufnahme von DualEnergy-Bildserien erlauben, wobei die Aufnahmetechnik mittels einem DSCTScanner und das Rapid-Kilovoltage-Switching die marktführenden Methoden sind. ${ }^{7}$

Bei einem DSCT-System, wie er mit dem Siemens Somatom Force der Firma Siemens Healthineers in unserer Studie verwendet wurde, sind zwei Röntgenstrahler um 90 Grad versetzt auf einer rotierenden Gantry installiert (Abb. 4). Gegenüber diesen liegen jeweils die dazugehörigen Detektoren. Bei der Untersuchung deckt ein Detektor das komplette Untersuchungsfeld ab, während der zweite auf ein kleineres, zentraleres Gebiet fokussiert ist. So kann eine Volumeneinheit (Voxel) simultan bestrahlt werden, ohne dabei eine signifikant höhere Strahlendosis zu benötigen. ${ }^{110,111}$ 

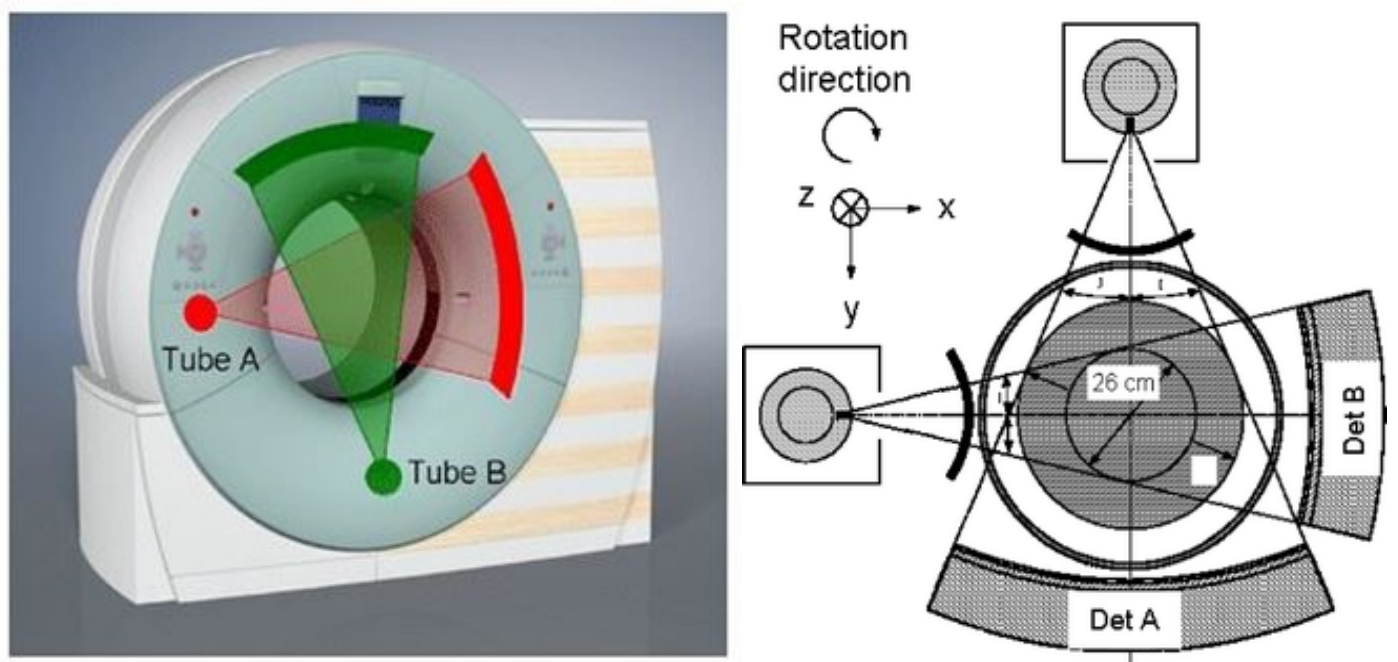

Abbildung 4: Darstellung eines Dual-Source-Computertomographen

Die beiden Röntgenröhren und die dazugehörigen Detektoren sind 90 Grad versetzt auf der Gantry angeordnet. Bei einer DECT werden beide Röhren mit unterschiedlichen Spannungen betrieben.

(Abbildung entnommen aus: Flohr TG et al. (2006): First performance evaluation of a dual-sourceCT (DSCT) system. $)^{110}$

Aus diesem Aufbau ergeben sich drei verschiedene Anwendungsmodi für einen DSCT-Scanner:

a. Der Betrieb von nur einer Röntgenröhre

b. Der Betrieb beider Röntgenröhren mit der gleichen Spannung

c. Der Betrieb beider Röntgenröhren mit unterschiedlichen Spannungen

Der erste Modus entspricht der Verwendung des Dual-Source-Computertomographen als konventionellen Single-Energy-Computertomographen, bei dem neben einem Röntgenstrahler auch nur ein Detektor verwendet wird. Für die beiden anderen Modi werden beide Strahlungsquellen und die dazugehörigen Detektoren benötigt.

Werden die beiden Röntgenröhren mit der gleichen Spannung verwendet handelt es sich streng genommen nicht um eine DECT, weshalb in diesem Fall von einer DSCT SECT gesprochen wird. Generell ermöglicht die Verwendung von zwei Systemen, welche synchron rotieren die Akquisition der Bilddatensätze in wesentlich kürzere Zeit, als es mit einem standardmäßigen SECT möglich ist. Eine 
Viertelumdrehung der Gantry ist dabei ausreichend um genügend Projektionsdaten für die Rekonstruktionen eines CT-Bildes zu generieren. ${ }^{112}$ Dadurch wird die Scandauer bis maximal 0,25s verkürzt und die zeitliche Auflösung der Untersuchung auf bis zu 66ms erhöht, was das Risiko von Bewegungsartefakten deutlich minimiert. Dadurch lassen sich vor allem Organe mit hoher Bewegungsaktivität präziser darstellen. ${ }^{113,114}$ Erstmalig angewandt wurde die DSCT im Rahmen der kardialen Bildgebung in Verbindung mit einer elektrokardiographisch-getriggerten Bildaquisition. ${ }^{114}$ Die sehr kurze Scandauer erlaubt es weitestgehend unabhängig von der Herzfrequenz des Patienten sehr robuste, diagnostisch verwertbare Schnittbilder zu gewinnen. Dies war früher so kaum umsetzbar, weshalb Patienten vor kardialen CT-Untersuchungen häufig Betablocker zur Senkung der Herzfrequenz verabreicht wurden. ${ }^{115,115}$ Die DSCT ist mittlerweile auf diesem Gebiet als gängiges Verfahren etabliert und wird beispielsweise in Großbritannien als diagnostisches Mittel der Wahl zur Suche nach koronaren Stenosen bei stabiler Angina pectoris empfohlen. ${ }^{114,116,117}$ Des Weiteren wird durch die reduzierte Untersuchungsdauer ermöglicht, die Geschwindigkeit des Tischvorschubs deutlich zu erhöhen, sodass die Strahlenbelastung für den Patienten - trotz Verwendung von zwei Röntgenröhren - reduziert werden kann. ${ }^{110}$ Auch Patienten mit akuter Atemnot profitieren von der DSCT, da gezeigt werden konnte, dass auch ohne entsprechendes Atemkommando qualitativ hochwertige Bildserien erzeugt werden können. ${ }^{118}$ Ein Vorteil ergibt sich daraus für die pädiatrische Bildgebung. Kleinkinder können kein Atemkommando befolgen weshalb die Reduzierung von Bewegungsartefakten hier einen noch höheren Stellenwert besitzt. ${ }^{119}$ Der Betrieb beider Röntgenröhren mit unterschiedlichen Energieniveaus als dritte Anwendungsoption stellt die DECT dar, welche im Rahmen dieser Studie verwendet wurde. Die Röhrenspannungen können dabei individuell angepasst werden. So wurden in dieser Studie die Röntgenröhren mit 90 Kilovolt (kV) und 150 kV betrieben wodurch insgesamt drei verschiedene Bilddatensätze gewonnen wurden. Eine Serie mit niedriger Spannung (90 kV), eine mit hoher Spannung (150 kV) und eine gemischte Serie, bei der die beiden Bildserien im Verhältnis 50-50 fusioniert werden und so der Bildeindruck einer 120 kV-Serie entsteht, die vergleichbar ist mit einer herkömmlichen MDCT-Aufnahme. ${ }^{112}$ Man kann unterscheiden zwischen harter (Röntgenspannung > $100 \mathrm{kV}$ ), mittelharter (Röntgenspannung 60 - 100 kV) und weicher Strahlung (Röntgenspannung 20 - 
60 kV). ${ }^{120}$ Für das Penetrations- bzw. Absorptionsverhalten des Durchstrahlten gilt: Harte Strahlung wird weniger absorbiert und penetriert das Gewebe besser, wohingegen mittelharte und weiche Strahlung das Gewebe weniger gut penetriert und mehr absorbiert wird. ${ }^{112}$

Die Bildentstehung bei radiologischen Aufnahmen basiert im Wesentlichen auf der Absorption der erzeugten Röntgenstrahlung im Gewebe. Diese beruht auf den gewebespezifischen, linearen Schwächungskoeffizienten, welche abhängig sind von der Quantenenergie, der Dicke und der chemischen Ordnungszahl $(Z)$ der durchstrahlten Materie. Zudem besteht eine Energieabhängigkeit, die vor allem durch den Photoeffekt und die Comptonstreuung zustande kommt. ${ }^{102,112}$ Der Photoeffekt beschreibt einen Vorgang der bei niedrigen Energien dominiert. Dabei trifft ein Photon klassischerweise auf ein Elektron der inneren Schale des Atoms, wobei die Energie des Photons auf das Elektron übertragen wird. Das Elektron wird anschließend aus der Schale herausgelöst (Ionisation) und emittiert. Dieser Effekt tritt vor allem dann auf, wenn die Photonenenergie nur geringfügig größer ist als die Bindungsenergie des Schalenelektrons; ist sie geringer, kann keine Interaktion auftreten. Gleichen sich die Photonenenergie und die Bindungsenergie, kommt es zu einer sprunghaften Zunahme der Schwächung der Röntgenstrahlung mit charakteristischen K-Kanten (Absorptionskante). ${ }^{102,112} \mathrm{Da}$ die Bindungsenergie der Schalenelektronen mit steigender Ordnungszahl zunimmt und der menschliche Körper zum größten Teil aus Sauerstoff $(Z=8)$, Stickstoff $(Z=7)$, Kohlenstoff $(Z=6)$ und Wasserstoff $(Z=1)$ besteht, werden K-Kanten seltener beobachtet. ${ }^{121}$

Die Comptonstreuung tritt insbesondere dann auf, wenn die Bindungsenergie der Schalenelektronen signifikant kleiner ist als die Photonenenergie, weshalb dieser Effekt bei höheren Energien dominiert. Die Comptonstreuung ist größtenteils unabhängig von der Ordnungszahl und betrifft meist die Elektronen, welche weit vom Atomkern entfernt liegen. Folglich ist sie proportional zur Elektronendichte, wodurch ebenso eine Proportionalität zur Massendichte des bestrahlten Materials besteht. ${ }^{122}$ Es kommt zum Auftreffen des Photons auf das Schalenelektron, wobei die Energie teilweise übertragen wird und in Folge dessen das Elektron aus der Schale herausgelöst wird. Anschließend entfernen sich das Photon und das Elektron in einem definierten Winkel voneinander. ${ }^{102,112}$ 
Die beschriebene Energieabhängigkeit wird bei der DECT genutzt, da durch das simultane Bestrahlen von Voxeln mit verschiedenen Energieniveaus Unterschiede im Absorption- bzw. Schwächungsverhalten gemessen werden, die sich in höheren oder niedrigeren CT-Werten niederschlagen. ${ }^{102}$ Dadurch ermöglicht die DECT eine wesentlich bessere Gewebedifferenzierung und bietet die Möglichkeit, die Zusammensetzung von Geweben zu untersuchen und sie zu charakterisieren. ${ }^{123}$

Das entstandene CT-Bild repräsentiert also vor allem die gemessenen Schwächungswerte im untersuchten Gewebe. Diese werden bei der Bildkonstruktion mathematisch in Graustufen-Werte (CT-Werte) umgesetzt und in Hounsfield-Einheiten (HU) angegeben. Dabei besteht ein linearer Zusammenhang zwischen dem Absorptionskoeffizienten und der Kernladungszahl des Gewebes, denn je höher die Abschwächung im Gewebe ist, desto höher ist auch der ermittelte CTWert. Für die Berechnung gilt folgende Gleichung:

$$
C T-\text { Wert }[H U]=1000 \cdot \frac{\mu_{\text {Materie }}-\mu_{\text {Wasser }}}{\mu_{\text {Wasser }}}
$$

Per Definition hat Luft den CT-Wert 0 HU (da $\mu$ Luft $=0$ ) und Wasser den Wert -1000 HU.

Bei der DECT werden jedem Gewebe aufgrund der Energieabhängigkeit zwei CT-Werte zugeordnet, wodurch im Vergleich zu einer konventionellen SECT die Möglichkeit für eine Gewebedifferenzierung und Materialcharakterisierung um eine Dimension erweitert wird. ${ }^{112,123}$

Die Gewebeanalyse kann anschließend anhand des Algorithmus zur Identifikation von drei Substanzen erfolgen. Bei der DECT entstehen für jedes Voxel ein Wert für die niedrigere Spannung $(90 \mathrm{kV})$ und ein Wert für die höhere Spannung (150 kV), die dann in ein Koordinatensystem eingetragen werden (Abb. 5) kann. Jedes zu untersuchende Voxel wird dabei in drei Basissubstanzen zerlegt, deren CT-Werte fest definiert sind oder durch entsprechende Referenzmessung validiert wurden. Das Voxel ist letztendliche eine spezifische Zusammensetzung der drei Basissubstanzen. Die Dekomposition erfolgt vektoriell. 112,121,123 


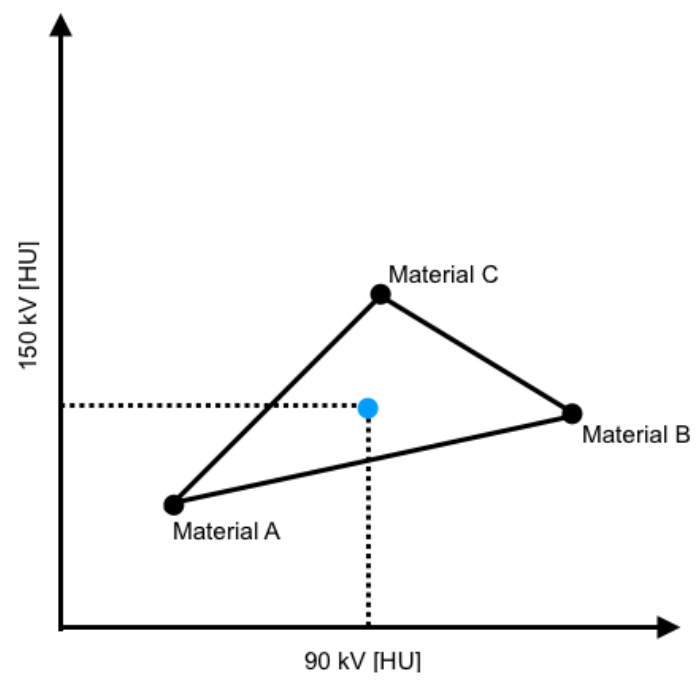

Abbildung 5: Vektorielle Dekomposition eines Voxels, modifiziert nach Sedlmair (2009) ${ }^{121}$

Die Dekomposition erfolgt in drei Basismaterialien.

Die klinischen Anwendungsgebiete der DECT sind mittlerweile recht breit gefächert. Sie wird unter anderem genutzt zur genaueren Beurteilung in der Onkologie (z.B. bei Lungenrundherden) ${ }^{124}$, bei CT-Angiographien zur Detektion von Embolien (insbesondere Lungenarterienembolien) ${ }^{125}$, Stenosen ${ }^{115,126,127}$ und abdomineller Aneurysmen ${ }^{128,129}$, zur Beurteilung von Uratkristallablagerungen in Gelenken bei Hyperurikämie ${ }^{130}$ und zur präzisen Charakterisierungen von Nierenund Gallensteinen. ${ }^{131,132}$ 


\subsubsection{Virtuelle Noncalcium Technik}

Die DECT erlaubt aufgrund der Akquisition von Bilddaten bei unterschiedlichen Röhrenspannungen ( $90 \mathrm{kV}$ und $150 \mathrm{kV}$ ) die Dekomposition anhand der oben beschriebenen Drei-Material-Dekomposition. Dadurch wird es möglich Substanzen wie Jod oder Kalzium, welche eine hohe Ordnungszahl und daher einen relevanten photoelektrischen Effekt besitzen, mithilfe entsprechender colour lookup tables (CLUT) farblich zu kodieren und mathematisch aus dem Bildeindruck zu subtrahieren.

In dieser Studie wurde für die Nachbearbeitung der DECT-Bilddatensätze („Postprocessing") die sogenannte virtuelle Noncalcium Technik verwendet, die erstmals 2010 von Pache et al. für den Nachweis von Knochenmarködemen beschrieben wurde. ${ }^{133}$ Die Bildanalyse basiert dabei auf einer Materialdekomposition in Knochensubstanz, rotes Knochenmark und gelbes Knochenmark. Mithilfe einer speziellen Software wird anschließend das Kalziumsignal virtuell aus dem Bildeindruck subtrahiert, was den Nachweis eines Knochenmarködems erlaubt und inzwischen in einer Vielzahl von Studien angewandt wurde. Guggenberger et al. (2012) beschäftigten sich mit dem Nachweis bei traumatischen Sprunggelenksverletzungen ${ }^{134}$, wohingegen Wang et al. (2013) und Bierry et al. (2014) in ihren Studien zeigen konnten, dass die Detektion von Knochenmarködemen auch im Rahmen von Wirbelkörperfrakturen möglich ist. ${ }^{135,136}$ Dabei sei allerdings gesagt, dass diese Daten auch mit gewissen Einschränkungen betrachtet werden müssen. Es konnte unter anderem gezeigt werden, dass unmittelbar subkortikal gelegene Ödeme nicht mit der nötigen Präzision dargestellt werden können und so einer Diagnostik entgehen können. ${ }^{133}$ Zudem berichten Wang et al. (2013) in ihrer Studie von einer gesteigerten Rate an falsch-positiven Befunden bei Patienten mit sklerotischen Wirbelsäulenveränderungen. ${ }^{135}$

Als weiteres Einsatzgebiet benutzten Reddy et al. (2014) und Baffour et al. (2019) die DECT VNCa-Technik bei Patienten mit Becken- und Hüftfrakturen und konnten dabei zeigen, dass vor allem der Nachweis kleiner, nicht verschobener Frakturen, die in der SECT übersehen werden können, akkurater ist. ${ }^{137,138}$ 
Anhand dessen ist davon auszugehen, dass insbesondere durch die Weiterentwicklung der Nachbearbeitungs-Algorithmen viele weitere Anwendungsgebiete für die VNCa-Technik möglich sind. ${ }^{7}$

Im Rahmen der vorliegenden Studie wurde der Algorithmus der VNCa-Rekonstruktionen unter Verwendung von zwei speziellen CLUT entsprechend modifiziert, um auf diesem Wege das Bandscheibengewebe farblich zu kodieren und es dadurch besser von den umliegenden Strukturen abgrenzbar zu machen und so eine sichere radiologische Diagnostik zu ermöglichen. 


\section{Material und Methoden}

Die lokale Ethik-Kommission des Fachbereichs Medizin der Johann Wolfgang Goethe-Universität Frankfurt am Main hat die vorliegende retrospektive Studie geprüft und genehmigt (Geschäfts-Nummer: 20-549). Eine gesonderte Zustimmung der Patienten war demzufolge nicht notwendig.

\subsection{Patientenselektion und Studiendesign}

Insgesamt wurden 87 potenzielle Patienten für die Aufnahme in unsere Studie in Betracht gezogen. Alle unterzogen sich aufgrund von akuten Schmerzen im unteren Rückenbereich einer klinisch indizierten und routinemäßig durchgeführten, kontrastmittelfreien DSCT DECT sowie einer kontrastmittelfreien MRT der Lendenwirbelsäule. Die Untersuchungen fanden zwischen März 2017 und Januar 2018 statt.

Der maximale Untersuchungsabstand zwischen DECT und MRT durfte dabei nicht länger als zwei Wochen betragen, um mögliche zeitlich bedingte Störfaktoren hinsichtlich der statistischen Korrelation zu minimieren (Anzahl $(n)=64)$. Es wurden Patienten ausgeschlossen, bei denen eine bekannte maligne Neoplasie der Wirbelsäule vorlag $(n=9)$. Außerdem wurden Patienten mit bekannter Spondylodiszitis $(n=4)$, dorsaler Instrumentierung $(n=5)$ oder interspinösen Platzhaltern (Spacer) $(n=5)$ nicht mit einbezogen. Zwei lumbale Bandscheiben konnten aufgrund von Blockwirbelbildungen nicht berücksichtigt werden, eine Bandscheibe wurde bei vorangegangener Nukleotomie ausgeschlossen.

Insgesamt wurden somit in der vorliegenden retrospektiven Studie 243 Bandscheiben der Lendenwirbelsäule (TH12 - S1) von $n=41$ Patienten ausgewertet. (Abb. 6). Davon waren $n=24$ (59\%) des weiblichen und $n=17$ (41\%) des männlichen Geschlechts. Die Alterspanne der Patienten lag bei 35 - 92 Jahren. Das Durchschnittsalter aller Teilnehmer war $68 \pm 14$ Jahren. Das durchschnittliche Alter der Frauen betrug, wie auch das der Männer, 68 Jahre. In Tabelle 2 sind weitere Charakteristika der Patientenpopulation dieser Studie aufgeführt.

Es wurden mehrere Ausschlusskriterien für die Teilnahme an der Studie festgelegt. Patienten, die absolute Kontraindikationen für die Durchführung einer DECT 
oder einer MRT hatten, konnten konsequenterweise nicht berücksichtigt werden. Somit galt hinsichtlich der DECT eine bekannte Schwangerschaft als Ausschlusskriterium. Mit Blick auf die MRT wurden keine Patienten mit Herzschrittmacher, implantiertem Defibrillator oder Cochlea-Implantaten berücksichtigt. Des Weiteren wurden Patienten exkludiert, welche zum Untersuchungszeitpunkt noch nicht volljährig waren.

\begin{tabular}{|c|c|c|}
\hline Charakteristik & Wert & Spannweiten \\
\hline Alter (y) & $68 \pm 14$ & $35-92$ \\
\hline Body-Mass-Index (BMI) (kg/m²) & $25 \pm 3$ & $17-34$ \\
\hline Weibliches Geschlecht, n (\%) & $24(59)$ & \\
\hline Alter $(y)$ & $68 \pm 16$ & $35-91$ \\
\hline BMI $\left(\mathrm{kg} / \mathrm{m}^{2}\right)$ & $27 \pm 5$ & $17-34$ \\
\hline Männliches Geschlecht, n (\%) & $17(41)$ & \\
\hline Alter $(y)$ & $68 \pm 12$ & $50-92$ \\
\hline BMI $\left(\mathrm{kg} / \mathrm{m}^{2}\right)$ & $23 \pm 3$ & $19-30$ \\
\hline $\begin{array}{l}\text { Anzahl der Patienten mit bekanntem lumbalen } \\
\text { Bandscheibenvorfall, } \mathrm{n}(\%)\end{array}$ & $6(15)$ & \\
\hline $\begin{array}{l}\text { Anzahl der Patienten mit bekannter Osteopo- } \\
\text { rose, n (\%) }\end{array}$ & $8(20)$ & \\
\hline $\begin{array}{l}\text { Anzahl der Patienten mit bekannter Skoliose, } \\
n(\%)\end{array}$ & $5(12)$ & \\
\hline
\end{tabular}

Tabelle 2: Charakterisierung der untersuchten Patientengruppe in dieser Studie

$\mathrm{n}=41$

Angaben, sofern nicht anders definiert, \pm Standardabweichung.

y (Jahre), kg (Kilogramm), m² (Quadratmeter), n (Anzahl) 
Einschlusskriterien: Alle Patienten, die sich einer Untersuchung mittels DECT und MRT unterzogen.

Zeitraum: März 2017 - Januar 2018

Zeitliches Intervall zwischen Untersuchungen maximal zwei Wochen

$$
\mathrm{n}=64
$$

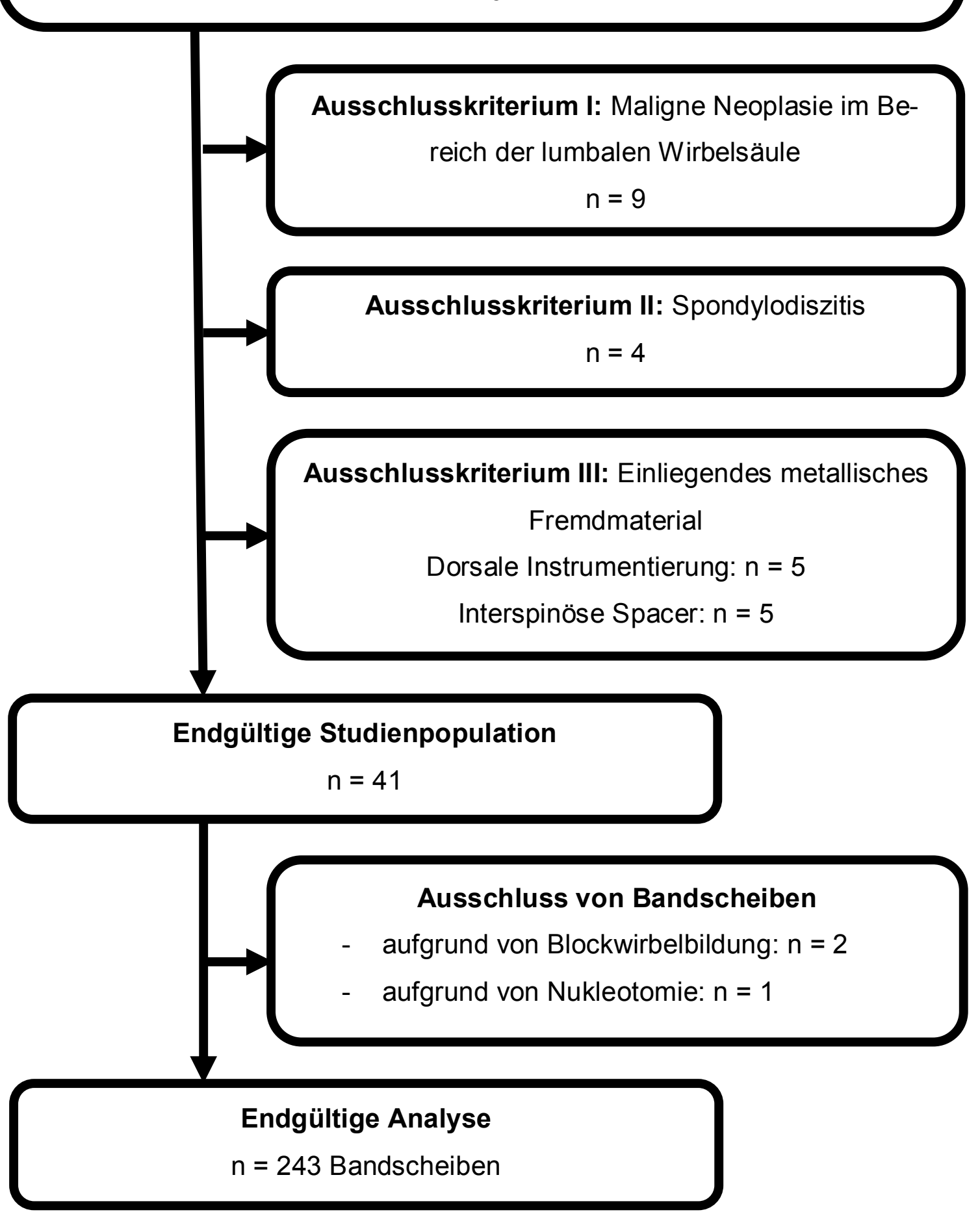

Abbildung 6: Flow-Chart zur Selektion der Studienpopulation 


\subsection{DECT Untersuchungsprotokoll}

Für die kontrastmittelfreien CT-Untersuchungen wurde in unserer Studie ein Dual-Source-Computertomograph der dritten Generation genutzt (Somatom Force; Siemens Healthineers, Forchheim, Germany). Es handelt sich bei dem Gerät um einen hochmodernen 2 x 192-Zeilen Dual-Source-Computertomographen, welcher seit November 2014 am Institut für Diagnostische und Interventionelle Radiologie des Universitätsklinikums Frankfurt am Main verwendet wird. Die beiden Röntgenröhren wurden im Rahmen der Untersuchungen bei verschiedenen Röhrenspannungen betrieben. Die Geräteeinstellungen sind in Tabelle 3 dargestellt.

\begin{tabular}{|l|c|c|}
\hline Parameter & Röhre A & Röhre B \\
\hline Röhrenspannung $[\mathrm{kV}]$ & 90 & Sn150 (mit 0,64-mm Zinn-Filter) \\
\hline Stromstärke [mAs] & 220 & 138 \\
\hline Rotationsdauer: $0,5 \mathrm{~s}$ & & \\
\hline Pitchfaktor: 0,6 & \\
\hline Kollimation: $128 \times 0.6 \mathrm{~mm}$ &
\end{tabular}

Tabelle 3: Untersuchungseinstellungen Dual-Source-Computertomograph

$\mathrm{kV}$ (Kilovolt), mm (Millimeter), mAs (Milliamperesekunden), s (Sekunden)

Die Patienten wurden bei allen Aufnahmen auf dem Rücken gelagert und die Bilderserien in kraniokaudaler Ausrichtung akquiriert.

Zur bestmöglichen Reduktion der Strahlenbelastung der Patienten wurde außerdem eine automatische, dämpfungsbasierte Röhrenstrommodulation (CARE Dose 4D; Siemens Healthineers) angewendet. Dabei wird der Röhrenstrom automatisch an die anatomischen Gegebenheiten des Patienten, wie Größe und Körperform, angepasst und fließt dadurch nicht kontinuierlich über die gesamte Untersuchungsdauer. 
Der durchschnittliche Volumen-CT-Dosisindikator \pm Standardabweichung (SD) lag bei 9.4 Milligray (mGy) $\pm 3,6$ (Spannweite: 3,0 - 13,3 mGy) und das durchschnittliche Dosis-Längen-Produkt betrug 321,4 mGy * $\mathrm{cm} \pm 64,5$ (Spannweite: $\left.86,6-789,1 \mathrm{mGy}{ }^{*} \mathrm{~cm}\right)$. 


\subsection{MRT Untersuchungsprotokoll}

Alle Patienten unterzogen sich einer kontrastmittelfreien MRT-Untersuchung der Lendenwirbelsäule, welche in unserer Studie mit einem 3,0-Tesla-System (Magnetom PrismaFit; Siemens Healthineers) und spezieller Oberflächenspule für Wirbelsäulen durchgeführt wurden. Es wurden insgesamt drei Bildsequenzen akquiriert. Eine Standard T1-gewichtete Spin-Echo, eine T2-gewichtete Fast SpinEcho sowie eine Turbo Inversion-Recovery Magnitude (TIRM). Alle Sequenzen wurden in sagittaler Ausrichtung generiert. Die T2-gewichtete Sequenz wurde zusätzlich auch in axialer Ebene erzeugt. Die gesammelten Parameter sind in Tabelle 4 zusammengefasst.

\begin{tabular}{|l|c|c|c|}
\hline Parameter & $\begin{array}{c}\text { T1-gewichtet } \\
\text { Spin-Echo }\end{array}$ & $\begin{array}{c}\text { T2-gewichtet } \\
\text { Fast-Spin-Echo }\end{array}$ & TIRM \\
\hline $\begin{array}{l}\text { Wiederholungszeit } \\
\text { (ms) }\end{array}$ & 650 & 4000 & 3500 \\
\hline Echo-Zeit (ms) & 10 & 89 & 39 \\
\hline Matrix-Größe & $288 \times 384$ & $358 \times 448$ & $388 \times 384$ \\
\hline Schichtdicke (mm) & 4 & 4 & 4 \\
\hline
\end{tabular}

Tabelle 4: Daten der Magnetresonanztomographie ms (Millisekunden), mm (Millimeter) 


\subsection{CT-Bildrekonstruktion und Nachverarbeitung}

Bei jeder durchgeführten CT-Untersuchung wurden drei verschiedenen Bilddatensätze akquiriert: $90 \mathrm{kV}$, Sn150 kV und eine gemischte Serie (Verhältnis 50:50), welche den Kontrasteigenschaften einer gewöhnlichen 120 kV-Sequenz entspricht und üblicherweise bei monoenergetischen CT-Aufnahmen entsteht. ${ }^{128}$ Bei der Rekonstruktion der Bilder unterscheidet man generell scharfe und weiche Faltungskerne, mithilfe derer man den Bildeindruck verändern kann. Scharfe Faltungskerne ermöglichen es Bilder mit hoher Bildschärfe zu erzeugen, was aber den Nachteil eines hohen Bildrauschens mit sich bringt. Umgekehrt verhält es sich bei der Verwendung weicher Faltungskerne. ${ }^{112}$ Die Rekonstruktionen in unserer Studie (axial, Schichtdicke: $2 \mathrm{~mm}$, Inkrement: $1 \mathrm{~mm}$; sagittal, Schichtdicke $2 \mathrm{~mm}$, Inkrement: $2 \mathrm{~mm}$ ) wurden mit einem speziellen mittelweichen Faltungskern (Qr40, Advanced Model-based Iterative Reconstruction [ADMIRE] level of 3) für Hoch- und Niedrigkilovolt-Serien generiert. Die Bildnachbearbeitung erfolgte mit einer handelsüblichen dreidimensionalen Workstation (syngo.via, Version VB10B; Siemens Healthineers) unter Verwendung eines Algorithmus, der die Unterscheidung zwischen Knochensubstanz sowie rotem und gelben Knochenmark zulässt. ${ }^{133,139}$ Dabei wurde ein VNCa-Rekonstruktionsalgorithmus verwendet, welcher für die Darstellung von Bandscheiben mittels folgenden Softwareeinstellungen optimiert wurde:

- CLUT für niedrige Energiewerte, Spektrum („spectrum“)

- CLUT für hohe Energiewerte, Graustufe („grayscale“)

- CT-Voreinstellung 1, Leber (,liver“)

- CT-Voreinstellung 2, Knochen („,bone“)

Nach dem subjektiven Bildeindruck wurde anschließend die Fensterung der Bildserien manuell angepasst. Die Fensterbreite wurde zwischen 750 und $800 \mathrm{HU}$ und das Fensterzentrum zwischen 45 und $150 \mathrm{HU}$ gewählt. Für die Bildanalyse wurden die axialen und sagittalen VNCa-Rekonstruktionen wie auch die Standard CT-Serien in das interne Bildarchivierungs- und Kommunikationssystem (Picture Archiving and Communication System (PACS)) übertragen. 


\subsection{Bildanalyse}

Die Bildanalyse wurde mit einer gängigen PACS Workstation (Centricity, Version 4.2; GE Healthcare, Solingen, Germany) durchgeführt. Um einen Referenzstandard festzulegen, analysierten in einem ersten Schritt zwei Fachärzte für Radiologie im Konsens die MRT-Untersuchungen in Bezug auf das Vorhandensein und den Grad eines lumbalen Bandscheibenvorfalls gemäß der Klassifikation der North American Spine Society, Version 2.0 (NASS) ${ }^{36}$ sowie der möglichen Affektion von Spinalnervenwurzeln nach dem Graduierungssystem von Pfirrmann et al. (2004). ${ }^{32}$ Beide Ärzte besitzen jeweils 20 beziehungsweise 32 Jahre Erfahrung in der muskuloskelettalen Bildgebung und wurden hinsichtlich klinischen und DECT-Informationen verblindet. Jeder lumbale Bandscheibenvorfall wurde von den Untersuchern entsprechend als Protrusion, Extrusion oder Sequestration klassifiziert. Zwischenwirbelscheiben, die lediglich eine breitbasige Vorwölbung (Bulging) aufwiesen, wurden dabei - ebenfalls entsprechend der Klassifikation der NASS - als nicht pathologisch bewertet. ${ }^{36}$ Hinsichtlich der Detektion einer Einklemmung von Spinalnervenwurzeln durch Bandscheibenmaterial, wurden alle Untersucher gebeten jegliche Affektion zu vermerken, unabhängig davon, ob dieser eine Bandscheibenherniation oder lediglich eine Bandscheibenvorwölbung zu Grunde lag. Beide Radiologen konnten die Fensterung selbstständig anpassen und besaßen die Möglichkeit frei durch die gesamten MRT-Bildserien zu scrollen. Anhand einer Fünf-Punkte-Bewertungsskala wurde zudem die Bildqualität, das Bildrauschen und die diagnostische Sicherheit bewertet. ${ }^{10}$ Dabei galt ein Punkt als schlechtestes und fünf Punkte als bestes Ergebnis. In Tabelle 5 sind die Bewertungskriterien mit den jeweilig zugeordneten Punktewerten dargestellt. 


\begin{tabular}{|l|l|l|}
\hline Bildqualität & Bildrauschen & $\begin{array}{l}\text { Diagnostische } \\
\text { Sicherheit }\end{array}$ \\
\hline 1 = inakzeptabel & $1=$ inakzeptabel & $\begin{array}{c}1=\text { nicht diagnostizier- } \\
\text { bar }\end{array}$ \\
\hline $2=$ unterdurchschnittlich & $2=$ unterdurchschnittlich & $2=$ sehr unsicher \\
\hline $3=$ durchschnittlich & $3=$ durchschnittlich & $\begin{array}{c}3=\text { Diagnose } \\
\text { wahrscheinlich }\end{array}$ \\
\hline $4=$ überdurchschnittlich & $4=$ überdurchschnittlich & $4=$ sehr sicher \\
\hline $5=$ exzellent & $5=$ exzellent & $5=$ absolut sicher \\
\hline
\end{tabular}

Tabelle 5: Bewertungskriterien für die Bildanalyse, modifiziert nach Notohamiprodjo et al., $2017^{10}$

Im Anschluss daran wurden die axialen und sagittalen DECT-Serien von zwei Fachärzten für Radiologie mit neun und sechs Jahren Erfahrung und drei Assistenzärzten der Radiologie (fünf, drei und drei Jahre Erfahrung) sowie einem Medizinstudenten kurz vor Studienabschluss mit einem Jahr Erfahrung analysiert. Alle Untersucher wurden im Hinblick auf die MRT-Ergebnisse, die Indikationen und die klinische Anamnese der Patienten verblindet. Zunächst wurden die konventionellen Graustufen-CT-Serien in zufälliger Reihenfolge präsentiert. Die Teilnehmer wurden gebeten, das Vorhandensein und den Grad aller lumbalen Bandscheibenvorfälle sowie die mögliche Beteiligung von Spinalnervenwurzeln zu evaluieren. Dabei sollte jede Bandscheibe jedes Patienten untersucht und analysiert werden. Erst nach acht Wochen wurden die farbkodierten VNCa-Rekonstruktionen beurteilt, um eine Ergebnisverzerrung aufgrund einer möglichen Erinnerung (recall bias) zu verhindern. Auch die VNCa-Rekonstruktionen wurden in zufälliger Reihenfolge gezeigt und es bestand während der Bildanalyse kein Zugang zu den Daten der Graustufen-CT. Die Gutachter konnten auch hier die Einstellungen der Fensterung beliebig ändern und besaßen die Möglichkeit, die gesamten Bildserien durchzublättern. Bewertet wurden wieder die Bildqualität, das Bildrauschen und die diagnostische Sicherheit anhand der bereits zuvor erwähnten Fünf-Punkte-Skala (Tabelle 5). 


\subsection{Statistische Analyse}

Die statistischen Analysen wurden mit speziellen Softwareprogrammen (SPSS Statistics for Windows, Version 23.0; IBM, Armonk NY und MedCalc for Windows, Version 13; MedCalc, Mariakerke, Belgien) durchgeführt. Zur Prüfung, ob die gesammelten Daten der zu Grunde liegenden Studie normalverteilt sind, wurde der Kolmogorow-Smirnov-Test angewandt. Dieser Test beruht darauf, dass die Verteilungsfunktion mit einer hypothetischen Verteilung - in unserem Fall der Normalverteilung - verglichen wird und eignet sich vor allem bei kleineren Stichprobengrößen. ${ }^{140}$ Die Variablen wurden mit dem Wilcoxon-Test für verbundene Stichproben auf statistisch signifikante Unterschiede getestet. Angegeben wurden diese als Mittelwerte \pm Standardabweichung. Das Signifikanzniveau wurde auf $5 \%$ festgelegt. Somit gilt für die berechneten P-Werte:

$$
\begin{array}{ll}
-P>0,05 & \text { keine Signifikanz } \\
-P<0,05 & \text { es besteht Signifikanz } \\
-P<0,01 & \text { es besteht hohe Signifikanz }
\end{array}
$$

Die Werte für die Sensitivität, die Spezifität, den positiven prädiktiven Wert (PPV), den negativen prädiktiven Wert (NPV) und die Genauigkeit wurden sowohl pro Patient als auch pro Bandscheibe berechnet. Das Clustering von Bandscheiben wurde für den einzelnen Untersucher mithilfe der von Genders et al. (2012) ${ }^{141}$ beschriebenen Methode berücksichtigt. Bei der Auswertung der gesammelten Werte aller Untersucher wurde dem Clustering auf Basis einer Kontingenztafel Rechnung getragen. Es wurde eine logistische Regressionsanalyse mit einem robusten Varianzschätzer verwendet. Um das Clustering der Bandscheiben pro Patient zu berücksichtigen wurden die Werte für Sensitivität, Spezifität, PPV und NPV zwischen den beiden CT-Algorithmen unter Verwendung verallgemeinerter Schätzgleichungen mit einer unabhängigen Korrelationsmatrix und robustem Varianzschätzer miteinander verglichen. Als statistisches Maß für die Übereinstimmung zwischen den einzelnen Untersuchern wurde das gewichtete Fleiss $\kappa$ entsprechend der Methodik von Landis und Koch (1977) ${ }^{142}$ angewendet und bewertet. 
Dabei gilt:

$\begin{array}{lll}-\kappa<0,20 & \text { slight } & \text { leichte Übereinstimmung } \\ -\kappa=0,21-0,40 & \text { fair } & \text { ausreichende Übereinstimmung } \\ -\kappa=0,41-0,60 & \text { moderate } & \text { mäßige Übereinstimmung } \\ -\kappa=0,61-0,80 & \text { substantial } & \text { gute Übereinstimmung } \\ -\kappa=0,81-1,00 & \text { almost perfect } & \text { exzellente Übereinstimmung }\end{array}$




\section{Ergebnisse}

Insgesamt wurden 243 Bandscheiben der Lendenwirbelsäule (Median pro Patient: 6; Spannweite: 5 - 6) von 41 Patienten (Durchschnittsalter 68 Jahre \pm 14 ; Spannweite: 35-92 Jahre) in die Studie eingeschlossen. Unter den 41 Patienten waren 24 Frauen (Durchschnittsalter: $68 \pm 16$ Jahre; Spannweite: 35-91 Jahre) und 17 Männer (Durchschnittsalter: $68 \pm 12$ Jahre; Spannweite: 50-92 Jahre).

Die Auswertung der MRT, die als Referenzstandard diente, ergab 112 lumbale Bandscheibenvorfälle, was einen Anteil von 46\% (112 von 243 Bandscheiben) ergibt. Dabei lag der Median pro Patient bei drei mit einer Spannweite von 0 - 6. In 29 Fällen wurde zudem eine Affektion von Spinalnervenwurzeln diagnostiziert. In Anlehnung an die Klassifikation der NASS wurden die lumbalen Bandscheibenvorfälle wie folgt gewertet: 58 Protrusionen (24\%), 51 Extrusionen (21\%) sowie drei Sequester (1\%). ${ }^{36}$ Der Abstand zwischen der DECT- und der MRT-Untersuchung betrug im Durchschnitt vier Tage (Spannweite: 0 - 13 Tage).

\subsection{Diagnostische Genauigkeit pro Patient}

Bezogen auf den einzelnen Patienten zeigte die Analyse für den Nachweis von Protrusionen der lumbalen Bandscheiben insgesamt eine höhere Sensitivität (169 von 174 [97\%] vs. 146 von 174 [84\%], Spezifität (53 von 72 [74\%] vs. 25 von 72 [35\%], PPV (169 von 188 [90\%] vs. 146 von 193 [76\%]), NPV (53 von 58 [91\%] vs. 25 von 53 [47\%]) und Genauigkeit (222 von 246 [90\%] vs. 171 von 246 [70\%]) der farbkodierten VNCa-Rekonstruktionen im Vergleich zu standardmäßigen CT-Bildserien (alle Vergleiche, $P<0,001$ ). Somit sind die Ergebnisse hoch signifikant.

Abbildung 7 demonstriert beispielhaft die vereinfachte Detektion von lumbalen Bandscheibenvorfällen unter Verwendung der farbkodierten VNCa-Rekonstruktionen im Vergleich zur herkömmlichen Graustufen-CT. 

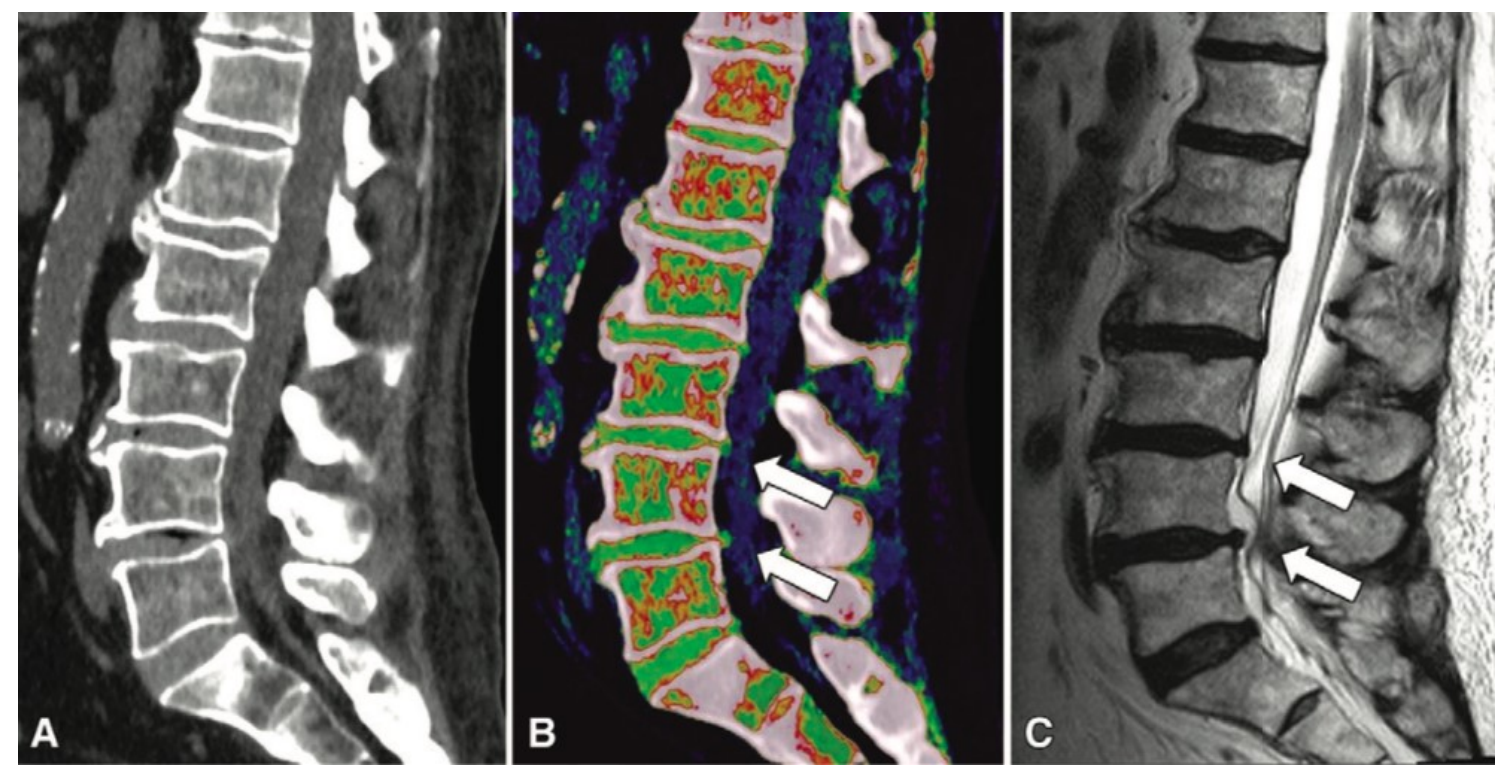

Abbildung 7: Bildgebung eines 65-jährigen Mannes

Der Patient stellte sich nach dem Hochheben schwerer Taschen mit akuten, starken Schmerzen im unteren Rückenbereich vor.

A: In der standardmäßigen, kontrastmittelfreien CT konnten die lumbalen Bandscheibenprotrusionen im Bereich L3/L4 und L4/L5 initial nicht sicher dargestellt werden.

B: Beide lumbalen Bandscheibenprotrusionen (Pfeile) wurden, nach der Rekonstruktion der sagittalen VNCa-Bildserien, welche für die Analyse von Bandscheiben optimiert wurde, von allen Untersuchern der Studie diagnostiziert.

C: Die zusätzlich durchgeführte kontrastmittelfreie MRT bestätigte die Diagnose in der sagittalen T2-gewichteten Bildserie.

Auch für den Nachweis von Affektionen/Komprimierungen der Spinalnervenwurzeln zeigten die farbigen VNCa-Rekonstruktionen insgesamt eine signifikant höhere Sensitivität (92 von 96 [96\%] vs. 82 von 96 [85\%], Spezifität (143 von 150 [95\%] vs. 130 von 150 [87\%], PPV (92 von 99 [93\%] vs. 82 von 102 [80\%]), NPV (143 von 147 [97\%] vs. 130 von 143 [90\%]) und Genauigkeit (235 von 246 [96\%] vs. 212 von 246 [86\%]) im Vergleich zu standardmäßigen CT-Bildserien (alle Vergleiche, $P<0,001$ ). Die Ergebnisse sind in Tabelle 6 zusammengefasst.

Die Übereinstimmung zwischen den Untersuchern hinsichtlich des Nachweises einer lumbalen Bandscheibenprotrusion zeigte signifikante Ergebnisse. Sie war "gut" für die VNCa-Rekonstruktionen und "mäßig" für die standardmäßigen CTBildserien ( $\kappa=0,75$ vs. 0,46 ). Bezogen auf die Diagnostik einer lumbalen Bandscheibenextrusion war die Übereinstimmung "gut" für VNCa und „mäßig“ für 
Standard-CT ( $\kappa=0,69$ vs. 0,41). Für die Detektion eines lumbalen Bandscheibensequesters war sie „exzellent“ (VNCa) und „mäßig“ (Standard-CT) $(\kappa=0,94$ vs. 0,41) und für die Beurteilung einer Beteiligung von Spinalnervenwurzeln „exzellent“ (VNCa) und „mäßig“ (Standard-CT) ( $\kappa=0,87$ vs. 0,56) (alle Vergleiche, $P<0,001)$. 


\begin{tabular}{|c|c|c|c|c|c|}
\hline & Sensitivität (\%) & Spezifität (\%) & PPV (\%) & NPV (\%) & Genauigkeit (\%) \\
\hline \multicolumn{6}{|c|}{ Protrusion } \\
\hline Standard-CT & $\begin{array}{c}146 / 175(84) \\
{[80 ; 88]}\end{array}$ & $\begin{array}{c}25 / 72(35) \\
{[24 ; 47]}\end{array}$ & $\begin{array}{c}146 / 193(76) \\
{[74 ; 81]}\end{array}$ & $\begin{array}{c}25 / 53(47) \\
{[27 ; 63]}\end{array}$ & $\begin{array}{c}171 / 246(70) \\
{[59 ; 82]}\end{array}$ \\
\hline VNCa & $\begin{array}{c}169 / 174(97) \\
{[93 ; 99]}\end{array}$ & $\begin{array}{c}53 / 72(74) \\
{[62 ; 83]}\end{array}$ & $\begin{array}{c}169 / 188(90) \\
{[86 ; 93]}\end{array}$ & $\begin{array}{c}53 / 58(91) \\
{[82 ; 96]}\end{array}$ & $\begin{array}{c}222 / 246(90) \\
{[84 ; 96]}\end{array}$ \\
\hline \multicolumn{6}{|c|}{ Extrusion } \\
\hline Standard-CT & $\begin{array}{c}\text { 68/156 (44) } \\
{[36 ; 52]}\end{array}$ & $\begin{array}{c}77 / 90(86) \\
{[78 ; 91]}\end{array}$ & $\begin{array}{c}68 / 81(84) \\
{[75 ; 90]}\end{array}$ & $\begin{array}{c}77 / 165(47) \\
{[43 ; 51]}\end{array}$ & $\begin{array}{c}145 / 246(59) \\
{[42 ; 75]}\end{array}$ \\
\hline VNCa & $\begin{array}{c}135 / 156(87) \\
{[77 ; 92]}\end{array}$ & $\begin{array}{c}79 / 90(88) \\
{[79 ; 94]}\end{array}$ & $\begin{array}{c}135 / 146(93) \\
{[87 ; 97]}\end{array}$ & $\begin{array}{c}\text { 79/100 (79) } \\
{[70 ; 86]}\end{array}$ & $\begin{array}{c}214 / 246(87) \\
{[80 ; 92]}\end{array}$ \\
\hline \multicolumn{6}{|c|}{ Sequester } \\
\hline Standard-CT & $\begin{array}{c}4 / 18(22) \\
{[6 ; 48]}\end{array}$ & $\begin{array}{c}217 / 218(95) \\
{[92 ; 98]}\end{array}$ & $\begin{array}{c}4 / 15(27) \\
{[11 ; 51]}\end{array}$ & $\begin{array}{c}217 / 231(94) \\
{[92 ; 95]}\end{array}$ & $\begin{array}{c}221 / 246(90) \\
{[85 ; 95]}\end{array}$ \\
\hline VNCa & $18 / 18$ (100) & $\begin{array}{c}227 / 228(100) \\
{[96 ; 100]}\end{array}$ & $\begin{array}{c}18 / 19(95) \\
{[93 ; 98]}\end{array}$ & $227 / 227(100)$ & $\begin{array}{c}245 / 246(100) \\
{[99 ; 100]}\end{array}$ \\
\hline \multicolumn{6}{|c|}{ Affektion Spinalnervenwurzel } \\
\hline Standard-CT & $\begin{array}{c}82 / 96(85) \\
{[77 ; 92]}\end{array}$ & $\begin{array}{c}130 / 150(87) \\
{[80 ; 92]}\end{array}$ & $\begin{array}{c}82 / 102(80) \\
{[73 ; 87]}\end{array}$ & $\begin{array}{c}130 / 143(90) \\
{[85 ; 94]}\end{array}$ & $\begin{array}{c}212 / 246(86) \\
{[81 ; 90]}\end{array}$ \\
\hline VNCa & $\begin{array}{c}92 / 96(96) \\
{[90 ; 99]}\end{array}$ & $\begin{array}{c}143 / 150(95) \\
{[91 ; 98]}\end{array}$ & $\begin{array}{c}92 / 99(93) \\
{[86 ; 96]}\end{array}$ & $\begin{array}{c}143 / 147(97) \\
{[93 ; 99]}\end{array}$ & $\begin{array}{c}\text { 235/246 (96) } \\
{[90 ; 99]}\end{array}$ \\
\hline
\end{tabular}

Tabelle 6: Diagnostische Genauigkeit der Standard-CT und farbkodierter VNCa-Rekonstruktionen pro Patient

Die MRT diente als Referenzstandard. Die Nenner zeigen die Gesamtzahl bezogen auf den jeweiligen Messparameter. Daten in eckigen Klammern sind 95\%-Konfidenzintervalle. 


\subsection{Diagnostische Genauigkeit pro Bandscheibe}

Verglichen mit den standardmäßigen CT-Bildserien zeigten die farbkodierten VNCa-Rekonstruktionen, auch unter Berücksichtigung der Clusterbildung, generell eine signifikant höhere Sensitivität (612 von 672 [91\%] vs. 534 von 672 [80\%]), Spezifität (723 von 786 [92\%] vs. 655 von 786 [85\%]), PPV (612 von 675 [91\%] vs. 534 von 655 [82\%]), NPV (723 von 783 [92\%] vs. 665 von 803 [83\%]) und Genauigkeit (1335 von 1458 [92\%] vs. 1199 von 1458 [82\%]) für den Nachweis eines lumbalen Bandscheibenvorfalls (alle Vergleiche, $P<0,001$ ). Die Übereinstimmung der Untersucher war dabei „exzellent“ für die VNCa-Bilder $(\kappa=0,82)$ und „gut" für die Standard-CT $(\kappa=0,67)(P<0,001)$.

Unter Bezugnahme auf die Klassifikation der NASS ${ }^{36}$ ergab die statistische Analyse, verglichen mit der Standard-CT, in der Gesamtheit eine signifikant höhere Sensitivität (288 von 348 [83\%] vs. 247 von 348 [71\%]), Spezifität (986 von 1110 [89\%] vs. 845 von 1110 [76\%]), PPV (288 von 412 [70\%] vs. 247 von 512 [48\%]), NPV (986 von 1046 [94\%] vs. 845 von 946 [89\%]) und Genauigkeit (1274 von 1458 [87\%] vs. 1092 von 1458 [75\%]) für die Detektion einer lumbalen Bandscheibenprotrusion bei Verwendung der VNCa-Rekonstruktionen unter Beachtung der Clusterbildung im Vergleich zur herkömmlichen Graustufen-CT (alle Vergleiche, $P<0,001)$. Tabelle 7 zeigt die gesammelten Ergebnisse.

Die Übereinstimmung zwischen den Untersuchern war „exzellent“ für die VNCaBilder $(\kappa=0,81)$, jedoch nur „mäßig“ für die Standard-CT $(\kappa=0,48)(P<0,001)$. Auch für die Detektion einer Affektion bzw. Komprimierung von Spinalnervenwurzeln zeigten die rekonstruierten VNCa-Bildserien im Allgemeinen eine signifikant höhere Sensitivität (160 von 174 [92\%] vs. 119 von 174 [68\%]), Spezifität (1261 von 1284 [98\%] vs. 1233 von 1284 [96\%]), PPV (160 von 183 [87\%] vs. 119 von 179 [70\%]), NPV (1261 von 1275 [99\%] vs. 1233 von 1288 [96\%]), und Genauigkeit (1421 von 1458 [97\%] vs. 1352 von 1458 [93\%]). Auch in dieser Analyse wurde eine Clusterbildung berücksichtigt (alle Vergleiche $P<0,001$ ). Die Übereinstimmung zwischen den Untersuchern war in diesem Fall "gut“ für die VNCaBilder $(\kappa=0,79)$ und „mäßig“ für Standard-CT ( $\kappa=0,46)$. Abbildung 8 zeigt beispielhaft wie mithilfe der verwendeten VNCa-Rekonstruktionen eine erleichterte Beurteilung der Affektion einer Spinalnervenwurzel möglich ist. 


\begin{tabular}{|c|c|c|c|c|c|}
\hline & Sensitivität ( $\%)$ & Spezifität (\%) & PPV (\%) & NPV (\%) & Genauigkeit (\%) \\
\hline \multicolumn{6}{|c|}{ Protrusion } \\
\hline Standard-CT & $\begin{array}{c}247 / 348(71) \\
{[64 ; 81]}\end{array}$ & $\begin{array}{c}845 / 1110(76) \\
{[70 ; 83]}\end{array}$ & $\begin{array}{c}247 / 512(48) \\
{[38 ; 57]}\end{array}$ & $\begin{array}{c}845 / 946(89) \\
{[83 ; 94]}\end{array}$ & $\begin{array}{c}1092 / 1458(75) \\
{[66 ; 85]}\end{array}$ \\
\hline VNCa & $\begin{array}{c}288 / 348(83) \\
{[74 ; 94]}\end{array}$ & $\begin{array}{c}986 / 1110(89) \\
{[82 ; 96]}\end{array}$ & $\begin{array}{c}288 / 412(70) \\
{[60 ; 79]}\end{array}$ & $\begin{array}{c}986 / 1046(94) \\
{[89 ; 97]}\end{array}$ & $\begin{array}{c}1274 / 1458(87) \\
{[79 ; 95]}\end{array}$ \\
\hline \multicolumn{6}{|c|}{ Extrusion } \\
\hline Standard-CT & $\begin{array}{c}95 / 306(31) \\
{[22 ; 43]}\end{array}$ & $\begin{array}{c}1120 / 1252(97) \\
{[94 ; 99]}\end{array}$ & $\begin{array}{c}95 / 127(75) \\
{[66 ; 86]}\end{array}$ & $\begin{array}{c}\text { 1120/1331 (84) } \\
{[75 ; 94]}\end{array}$ & $\begin{array}{c}1215 / 1458(83) \\
{[74 ; 92]}\end{array}$ \\
\hline VNCa & $\begin{array}{c}255 / 306(83) \\
{[77 ; 92]}\end{array}$ & $\begin{array}{c}1126 / 1152(98) \\
{[96 ; 100]}\end{array}$ & $\begin{array}{c}255 / 281(91) \\
{[82 ; 97]}\end{array}$ & $\begin{array}{c}1126 / 1177(96) \\
{[90 ; 100]}\end{array}$ & $\begin{array}{c}1387 / 1458(95) \\
{[90 ; 99]}\end{array}$ \\
\hline \multicolumn{6}{|c|}{ Sequester } \\
\hline Standard-CT & $\begin{array}{c}4 / 18(22) \\
{[11 ; 34]}\end{array}$ & $\begin{array}{c}1371 / 1440(95) \\
{[90 ; 99]}\end{array}$ & $\begin{array}{c}4 / 73(6) \\
{[2 ; 11]}\end{array}$ & $\begin{array}{c}1371 / 1385(99) \\
{[97 ; 100]}\end{array}$ & $\begin{array}{c}1375 / 1458(94) \\
{[88 ; 98]}\end{array}$ \\
\hline VNCa & $18 / 18(100)$ & $\begin{array}{c}1438 / 1440(100) \\
{[98 ; 100]}\end{array}$ & $\begin{array}{c}18 / 20(90) \\
{[80 ; 97]}\end{array}$ & $1438 / 1438(100)$ & $\begin{array}{c}1448 / 1458(99) \\
{[97 ; 100]}\end{array}$ \\
\hline
\end{tabular}

Tabelle 7: Diagnostische Genauigkeit von Standard-CT und farbkodierten VNCa-Rekonstruktionen zur Klassifikation von lumbalen Bandscheibenvorfällen pro Bandscheibe

Die Unterteilung von lumbalen Bandscheibenvorfällen hinsichtlich Protrusion, Extrusion und einer Sequestration erfolgte nach der Klassifikation für lumbale Bandscheibenpathologien der North American Spine Society ${ }^{36}$. Die MRT diente dabei als Referenzstandard unter Berücksichtigung der Clusterbildung. Für die korrekte Klassifizierung eines lumbalen Bandscheibenvorfalls ergab die statistische Analyse im Vergleich zu Standard-CT-Bildserien eine verbesserte diagnostische Genauigkeit zugunsten der VNCa-Rekonstruktionen.

Die Nenner zeigen die Gesamtzahl bezogen auf den jeweiligen Messparameter. Daten in eckigen Klammern sind 95\%-Konfidenzintervalle. 

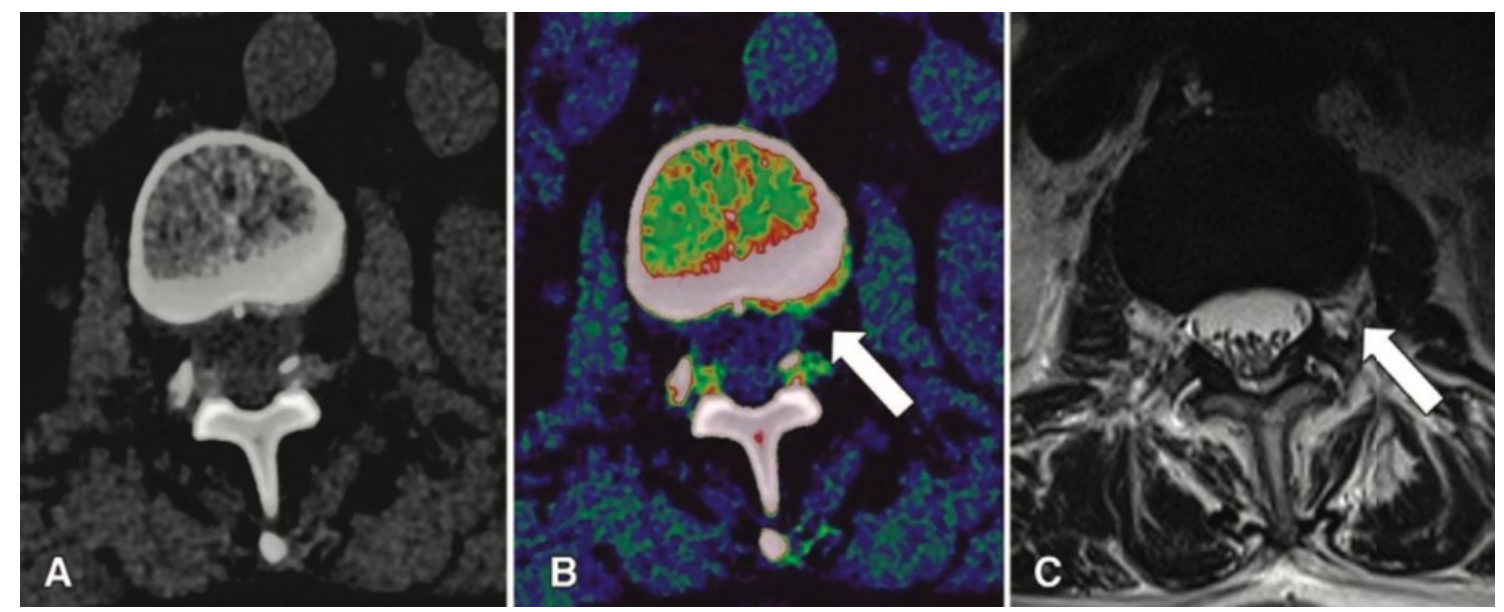

Abbildung 8: Bildgebung einer 62-jährigen Frau

Die Patientin, die unter einer bekannten Osteoporose leidet, stellte sich mit Schmerzen im unteren Rückenbereich, Parästhesien im linken Unterschenkel sowie eines kompletten Ausfalls des linksseitigen Musculus extensor hallucis longus vor.

A: Die Einklemmung der linken Spinalnervenwurzel durch die vorgewölbte Bandscheibe L4/L5 wurde unter Verwendung der kontrastmittelfreien, axialen CT-Untersuchung von 4 / 6 Untersuchern nicht erkannt.

B: Die Pfeilspitze zeigt eine Einklemmung der Spinalnervenwurzel zweiten Grades (Klassifikation nach Pfirmann et al. (2004)) 32. Die Diagnose wurde unter Zuhilfenahme der rekonstruierten axialen farbkodierten VNCa-Bildserien von allen Untersuchern gestellt.

C: Das Ausmaß der Einklemmung der Spinalnervenwurzel wurde durch eine zusätzlich durchgeführte MRT-Untersuchung mittels axialer T2-gewichteter Bildserien bestätigt. Die Pfeilspitze zeigt das betroffene Areal.

Vergleicht man die Ergebnisse aller sechs CT-Untersucher, so zeigen die Ergebnisse, dass durch die Verwendung der VNCa-Rekonstruktionen der unerfahrenste Untersucher (ein Jahr Erfahrung) die deutlichste Verbesserung hinsichtlich der diagnostischen Genauigkeit erreichte. Unter Berücksichtigung der Messparameter erzielte der genannte Untersucher eine signifikant höhere Sensitivität (101 von 112 [90\%] vs. 70 von 112 [63\%]), Spezifität (118 von 131 [90\%] vs. 98 von 131 [75\%]), PPV (101 von 114 [89\%] vs. 70 von 103 [68\%]), NPV (118 von 129 [92\%] vs. 98 von 140 [70\%]) und Genauigkeit (219 von 243 [90\%] vs. 168 von 243 [69\%] für den Nachweis einer lumbalen Bandscheibenherniation mittels VNCa-Rekonstruktionen (Abb. 9) (alle Vergleiche, $P<0,001$ ). Auch für die Detektion einer Beteiligung der Spinalnervenwurzeln erzielte er im Allgemeinen eine signifikant höhere Sensitivität (28 von 29 [97\%] vs. 19 von 29 [66\%]), Spezifität (208 von 214 [97\%] vs. 199 von 214 [93\%]), PPV (28 von 34 [82\%] vs. 
19 von 34 [56\%]), NPV (210 von 213 [99\%] vs. 199 von 209 [95\%]) und Genauigkeit (236 von 243 [97\%] vs. 218 von 243 [90\%]) (Abb. 10) (alle Vergleiche, $P<$ $0,001)$.

VNCa-Rekonstruktionen

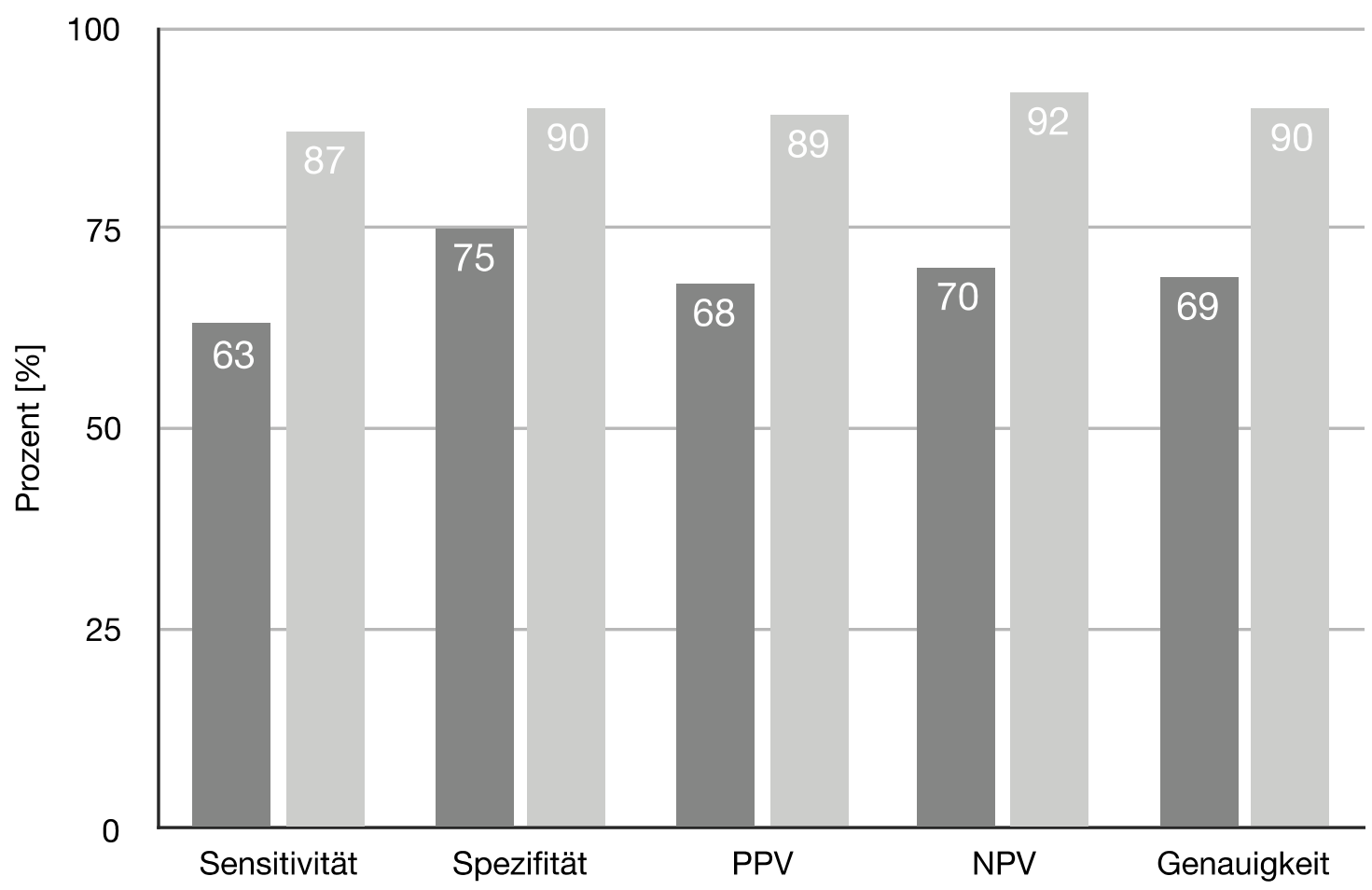

Abbildung 9: Vergleich der Studienparameter zum Nachweis einer lumbalen Bandscheibenherniation

Das Säulendiagramm demonstriert die Verbesserung des unerfahrensten Untersuchers bei Verwendung der VNCa-Rekonstruktionen für die Beurteilung einer lumbalen Bandscheibenherniation. 


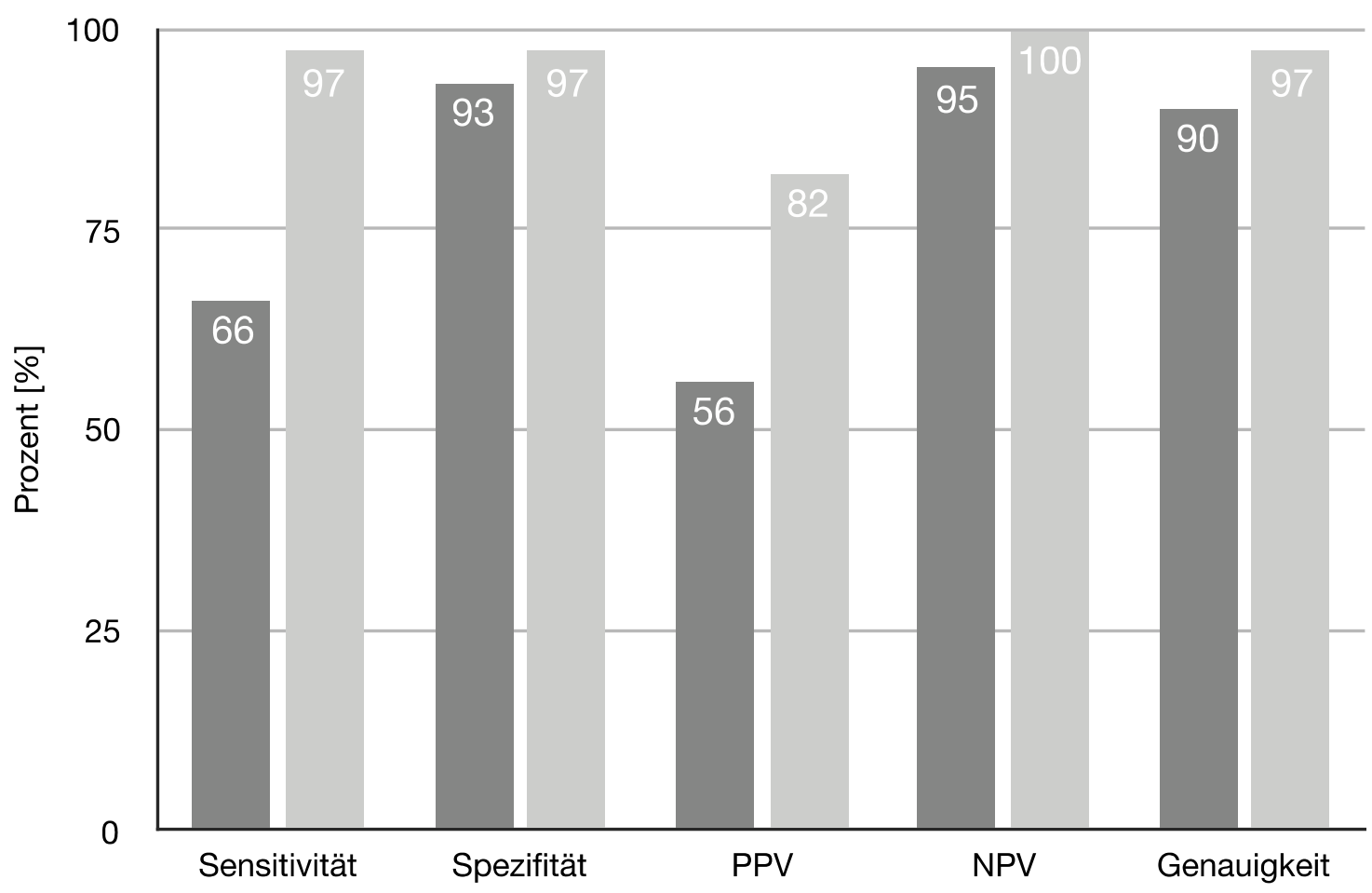

Abbildung 10: Vergleich der Studienparameter zum Nachweis einer Beteiligung von Spinalnervenwurzeln

Das Säulendiagramm demonstriert die Verbesserung des unerfahrensten Untersuchers bei Verwendung der VNCa-Rekonstruktionen für die Beurteilung einer Affektion von Spinalnervenwurzeln im Rahmen einer lumbalen Bandscheibenherniation.

Der erfahrenste Untersucher war mithilfe der rekonstruierten VNCa-Bildserien in der Lage eine diagnostische Leistungsfähigkeit zu erzielen, die in der Summe nicht nur der Graustufen-CT deutlich überlegen ist, sondern vergleichbar ist mit der MRT. Die Messparameter zeigten hier eine Sensitivität von 96\% (107 von 112), Spezifität von 95\% (124 von 131), PPV von 94\% (107 von 114), NPV von $96 \%$ (124 von 129) und eine Genauigkeit von 95\% (231 von 243). Alle anderen Untersucher konnten, verglichen mit der Standard-CT, durch Verwendung der VNCa-Rekonstruktionen sowohl für die Detektion eines lumbalen Bandscheibenvorfalls, als auch für den Nachweis einer Affektion von Spinalnervenwurzeln eine signifikant höhere Gesamtsensitivität, Spezifität, PPV, NPV und Genauigkeit (alle Vergleiche, $P<0,001)$ erzielen. In den Tabellen 8 und 9 sind die gesammelten Daten zusammengefasst. 


\begin{tabular}{|c|c|c|c|c|c|}
\hline & Sensitivität (\%) & Spezifität (\%) & PPV (\%) & NPV (\%) & $\begin{array}{c}\text { Genauigkeit } \\
(\%)\end{array}$ \\
\hline \multicolumn{6}{|c|}{ Durchschnitt } \\
\hline Standard-CT & $\begin{array}{c}534 / 672(80) \\
{[76 ; 83]}\end{array}$ & $\begin{array}{c}665 / 786(85) \\
{[82 ; 87]}\end{array}$ & $\begin{array}{c}534 / 655(82) \\
{[79 ; 84]}\end{array}$ & $\begin{array}{c}665 / 804(83) \\
{[81 ; 85]}\end{array}$ & $\begin{array}{c}1199 / 1458(82) \\
{[75 ; 86]}\end{array}$ \\
\hline VNCa & $\begin{array}{c}612 / 672(91) \\
{[89 ; 93]}\end{array}$ & $\begin{array}{c}\text { 723/786 (92) } \\
{[90 ; 94]}\end{array}$ & $\begin{array}{c}612 / 675(91) \\
{[89 ; 93]}\end{array}$ & $\begin{array}{c}723 / 783(92) \\
{[90 ; 94]}\end{array}$ & $\begin{array}{c}1335 / 1458(92) \\
{[88 ; 96]}\end{array}$ \\
\hline \multicolumn{6}{|c|}{ Untersucher 1} \\
\hline Standard-CT & $\begin{array}{c}\text { 70/112 (63) } \\
\text { [52; 71] }\end{array}$ & $\begin{array}{c}98 / 131(75) \\
{[67 ; 90]}\end{array}$ & $\begin{array}{c}70 / 103(68) \\
{[70 ; 85]}\end{array}$ & $\begin{array}{c}98 / 140(70) \\
{[67 ; 77]}\end{array}$ & $\begin{array}{c}168 / 243(69) \\
{[62 ; 76]}\end{array}$ \\
\hline VNCa & $\begin{array}{c}101 / 112(87) \\
{[85 ; 94]}\end{array}$ & $\begin{array}{c}118 / 131(90) \\
{[87 ; 94]}\end{array}$ & $\begin{array}{c}101 / 114(89) \\
{[86 ; 95]}\end{array}$ & $\begin{array}{c}118 / 129(92) \\
{[88 ; 94]}\end{array}$ & $\begin{array}{c}219 / 243(90) \\
{[84 ; 96]}\end{array}$ \\
\hline \multicolumn{6}{|c|}{ Untersucher 2} \\
\hline Standard-CT & $\begin{array}{c}92 / 112(82) \\
{[72 ; 84]}\end{array}$ & $\begin{array}{c}114 / 131(87) \\
{[84 ; 91]}\end{array}$ & $\begin{array}{c}92 / 109(84) \\
{[80 ; 88]}\end{array}$ & $\begin{array}{c}114 / 134(85) \\
{[73 ; 90]}\end{array}$ & $\begin{array}{c}206 / 243(85) \\
{[80 ; 90]}\end{array}$ \\
\hline VNCa & $\begin{array}{c}99 / 112(88) \\
\text { [83; 95] }\end{array}$ & $\begin{array}{c}119 / 131(91) \\
{[87 ; 95]}\end{array}$ & $\begin{array}{c}99 / 111(89) \\
{[82 ; 92]}\end{array}$ & $\begin{array}{c}119 / 132(90) \\
{[84 ; 95]}\end{array}$ & $\begin{array}{c}218 / 243(90) \\
{[86 ; 95]}\end{array}$ \\
\hline \multicolumn{6}{|c|}{ Untersucher 3} \\
\hline Standard-CT & $\begin{array}{c}\text { 100/112 (89) } \\
\text { [83; 91] }\end{array}$ & $\begin{array}{c}119 / 131(91) \\
{[84 ; 94]}\end{array}$ & $\begin{array}{c}100 / 112(89) \\
{[84 ; 92]}\end{array}$ & $\begin{array}{c}119 / 131(91) \\
{[85 ; 94]}\end{array}$ & $\begin{array}{c}219 / 243(90) \\
{[62 ; 76]}\end{array}$ \\
\hline VNCa & $\begin{array}{c}104 / 112(93) \\
{[87 ; 96]}\end{array}$ & $\begin{array}{c}\text { 125/131 (95) } \\
{[90 ; 98]}\end{array}$ & $\begin{array}{c}104 / 110(95) \\
{[89 ; 98]}\end{array}$ & $\begin{array}{c}125 / 133(94) \\
{[90 ; 97]}\end{array}$ & $\begin{array}{c}229 / 243(94) \\
{[90 ; 97]}\end{array}$ \\
\hline \multicolumn{6}{|c|}{ Untersucher 4} \\
\hline Standard-CT & $\begin{array}{c}99 / 112(88) \\
{[84 ; 91]}\end{array}$ & $\begin{array}{c}\text { 106/131 (81) } \\
{[75 ; 86]}\end{array}$ & $\begin{array}{c}99 / 124(80) \\
{[74 ; 85]}\end{array}$ & $\begin{array}{c}106 / 119(89) \\
{[81 ; 93]}\end{array}$ & $\begin{array}{c}205 / 243(84) \\
{[78 ; 88]}\end{array}$ \\
\hline VNCa & $\begin{array}{c}101 / 112(90) \\
{[87 ; 94]}\end{array}$ & $\begin{array}{c}115 / 131(88) \\
{[84 ; 92]}\end{array}$ & $\begin{array}{c}101 / 117(86) \\
{[80 ; 90]}\end{array}$ & $\begin{array}{c}\text { 115/126 (91) } \\
\text { [86; 95] }\end{array}$ & $\begin{array}{c}216 / 243(89) \\
\text { [85; 95] }\end{array}$ \\
\hline \multicolumn{6}{|c|}{ Untersucher 5} \\
\hline Standard-CT & $\begin{array}{c}78 / 112(70) \\
{[60 ; 78]}\end{array}$ & $\begin{array}{c}111 / 131(85) \\
{[80 ; 90]}\end{array}$ & $\begin{array}{c}78 / 98(80) \\
{[72 ; 87]}\end{array}$ & $\begin{array}{c}111 / 145(77) \\
{[70 ; 83]}\end{array}$ & $\begin{array}{c}189 / 243(78) \\
{[70 ; 85]}\end{array}$ \\
\hline $\mathrm{VNCa}$ & $\begin{array}{c}\text { 100/112 (89) } \\
\text { [83; 95] }\end{array}$ & $\begin{array}{c}\text { 122/131 (93) } \\
{[88 ; 96]}\end{array}$ & $\begin{array}{c}100 / 109(92) \\
{[86 ; 97]}\end{array}$ & $\begin{array}{c}122 / 134(91) \\
{[87 ; 95]}\end{array}$ & $\begin{array}{c}\text { 222/243 (91) } \\
\text { [86; 95] }\end{array}$ \\
\hline \multicolumn{6}{|c|}{ Untersucher 6} \\
\hline Standard-CT & $\begin{array}{c}95 / 112(85) \\
{[80 ; 90]}\end{array}$ & $\begin{array}{c}117 / 131(89) \\
{[81 ; 95]}\end{array}$ & $\begin{array}{c}95 / 109(87) \\
{[78 ; 93]}\end{array}$ & $\begin{array}{c}117 / 134(87) \\
{[83 ; 91]}\end{array}$ & $\begin{array}{c}212 / 243(87) \\
{[80 ; 90]}\end{array}$ \\
\hline VNCa & $\begin{array}{c}107 / 112(96) \\
{[92 ; 99]}\end{array}$ & $\begin{array}{c}124 / 131(95) \\
{[91 ; 98]}\end{array}$ & $\begin{array}{c}107 / 114(94) \\
{[90 ; 97]}\end{array}$ & $\begin{array}{c}124 / 129(96) \\
{[91 ; 98]}\end{array}$ & $\begin{array}{c}213 / 243(95) \\
{[91 ; 98]}\end{array}$ \\
\hline
\end{tabular}

Tabelle 8: Vergleich der diagnostischen Genauigkeit aller Untersucher zur Erkennung lumbaler Bandscheibenvorfälle pro Bandscheibe

Diagnostische Genauigkeit von Standard-CT und farbkodierten virtuellen VNCa-Rekonstruktionen zur Erkennung von lumbalen Bandscheibenvorfällen pro Bandscheibe. Die MRT-Diagnostik diente als Referenzstandard unter Berücksichtigung der Clusterbildung. Untersucher 1 hatte ein Jahr Erfahrung in der MSK-Bildgebung, Untersucher 2 hatte drei Jahre, Untersucher 3 hatte drei Jahre, Untersucher 4 hatte fünf Jahre und Untersucher 5 hatte insgesamt sechs Jahre Erfahrung. Die Nenner zeigen die Gesamtzahl bezogen auf den jeweiligen Messparameter. Daten in eckigen Klammern sind 95\%-Konfidenzintervalle. 


\begin{tabular}{|c|c|c|c|c|c|}
\hline & Sensitivität (\%) & Spezifität (\%) & PPV (\%) & NPV (\%) & $\begin{array}{c}\text { Genauigkeit } \\
(\%)\end{array}$ \\
\hline \multicolumn{6}{|c|}{ Durchschnitt } \\
\hline Standard-CT & $\begin{array}{c}119 / 174(68) \\
{[62 ; 76]}\end{array}$ & $\begin{array}{c}1233 / 1284(96) \\
{[94 ; 97]}\end{array}$ & $\begin{array}{c}119 / 170(70) \\
{[64 ; 76]}\end{array}$ & $\begin{array}{c}1233 / 1288(96) \\
{[92 ; 98]}\end{array}$ & $\begin{array}{c}1352 / 1458(93) \\
{[88 ; 95]}\end{array}$ \\
\hline VNCa & $\begin{array}{c}160 / 174(92) \\
{[87 ; 96]}\end{array}$ & $\begin{array}{c}1261 / 1284(98) \\
{[97 ; 99]}\end{array}$ & $\begin{array}{c}160 / 183(87) \\
{[82 ; 91]}\end{array}$ & $\begin{array}{c}1261 / 1275 \text { (99) } \\
{[98 ; 99]}\end{array}$ & $\begin{array}{c}1421 / 1458(97) \\
{[96 ; 99]}\end{array}$ \\
\hline \multicolumn{6}{|c|}{ Untersucher 1} \\
\hline Standard-CT & $\begin{array}{c}19 / 29(66) \\
{[53 ; 78]}\end{array}$ & $\begin{array}{c}199 / 214(93) \\
{[91 ; 96]}\end{array}$ & $\begin{array}{c}19 / 34(56) \\
{[44 ; 69]}\end{array}$ & $\begin{array}{c}\text { 199/209 (95) } \\
\text { [93; 98] }\end{array}$ & $\begin{array}{c}218 / 243(90) \\
{[84 ; 94]}\end{array}$ \\
\hline VNCa & $\begin{array}{c}28 / 29(97) \\
{[87 ; 99]}\end{array}$ & $\begin{array}{c}\text { 208/224 (97) } \\
{[94 ; 99]}\end{array}$ & $\begin{array}{c}\text { 28/34 (82) } \\
\text { [68; 99] }\end{array}$ & $\begin{array}{c}208 / 209(100) \\
{[97 ; 100]}\end{array}$ & $\begin{array}{c}\text { 236/243 (97) } \\
\text { [95; 99] }\end{array}$ \\
\hline \multicolumn{6}{|c|}{ Untersucher 2} \\
\hline Standard-CT & $\begin{array}{c}23 / 29(79) \\
{[70 ; 87]}\end{array}$ & $\begin{array}{c}\text { 208/214 (97) } \\
{[94 ; 99]}\end{array}$ & $\begin{array}{c}23 / 29(79) \\
{[71 ; 86]}\end{array}$ & $\begin{array}{c}\text { 208/214 (97) } \\
\text { [93; 98] }\end{array}$ & $\begin{array}{c}231 / 243(95) \\
{[90 ; 97]}\end{array}$ \\
\hline VNCa & $\begin{array}{c}26 / 29(90) \\
{[82 ; 99]}\end{array}$ & $\begin{array}{c}210 / 214(98) \\
{[95 ; 99]}\end{array}$ & $\begin{array}{c}26 / 30(87) \\
{[77 ; 91]}\end{array}$ & $\begin{array}{c}210 / 213(99) \\
{[96 ; 100]}\end{array}$ & $\begin{array}{c}236 / 243(97) \\
{[92 ; 100]}\end{array}$ \\
\hline \multicolumn{6}{|c|}{ Untersucher 3} \\
\hline Standard-CT & $\begin{array}{c}14 / 29(48) \\
{[35 ; 62]}\end{array}$ & $\begin{array}{c}\text { 209/214 (98) } \\
\text { [93; 99] }\end{array}$ & $\begin{array}{c}14 / 19(74) \\
{[64 ; 79]}\end{array}$ & $\begin{array}{c}\text { 209/224 (93) } \\
\text { [89; 96] }\end{array}$ & $\begin{array}{c}223 / 243(92) \\
{[88 ; 95]}\end{array}$ \\
\hline VNCa & $\begin{array}{c}26 / 29(90) \\
{[84 ; 97]}\end{array}$ & $\begin{array}{c}212 / 214(99) \\
{[95 ; 100]}\end{array}$ & $\begin{array}{c}\text { 26/28 (90) } \\
\text { [82; 96] }\end{array}$ & $\begin{array}{c}212 / 215(99) \\
{[97 ; 100]}\end{array}$ & $\begin{array}{c}238 / 243(92) \\
{[95 ; 100]}\end{array}$ \\
\hline \multicolumn{6}{|c|}{ Untersucher 4} \\
\hline Standard-CT & $\begin{array}{c}21 / 29(72) \\
{[59 ; 78]}\end{array}$ & $\begin{array}{c}\text { 205/214 (96) } \\
\text { [92; } 98]\end{array}$ & $\begin{array}{c}21 / 30(70) \\
\text { [62; 81] }\end{array}$ & $\begin{array}{c}\text { 205/213 (96) } \\
\text { [93; 98] }\end{array}$ & $\begin{array}{c}226 / 243(93) \\
{[88 ; 96]}\end{array}$ \\
\hline VNCa & $\begin{array}{c}26 / 29(90) \\
{[85 ; 96]}\end{array}$ & $\begin{array}{c}210 / 214(98) \\
{[96 ; 100]}\end{array}$ & $\begin{array}{c}26 / 30(87) \\
\text { [82; 93] }\end{array}$ & $\begin{array}{c}210 / 213(99) \\
{[97 ; 100]}\end{array}$ & $\begin{array}{c}236 / 243(97) \\
{[95 ; 100]}\end{array}$ \\
\hline \multicolumn{6}{|c|}{ Untersucher 5} \\
\hline Standard-CT & $\begin{array}{c}23 / 29(79) \\
{[70 ; 84]}\end{array}$ & $\begin{array}{c}207 / 214(97) \\
{[96 ; 99]}\end{array}$ & $\begin{array}{c}23 / 30(77) \\
{[60 ; 84]}\end{array}$ & $\begin{array}{c}207 / 213(97) \\
{[93 ; 99]}\end{array}$ & $\begin{array}{c}\text { 230/243 (95) } \\
\text { [88; 98] }\end{array}$ \\
\hline VNCa & $\begin{array}{c}27 / 29(93) \\
{[82 ; 99]}\end{array}$ & $\begin{array}{c}\text { 211/214 (99) } \\
{[98 ; 100]}\end{array}$ & $\begin{array}{c}27 / 130(90) \\
{[86 ; 95]}\end{array}$ & $\begin{array}{c}\text { 211/213 (99) } \\
{[98 ; 100]}\end{array}$ & $\begin{array}{c}238 / 243(98) \\
{[95 ; 100]}\end{array}$ \\
\hline \multicolumn{6}{|c|}{ Untersucher 6} \\
\hline Standard-CT & $\begin{array}{c}24 / 29(83) \\
{[64 ; 94]}\end{array}$ & $\begin{array}{c}\text { 205/214 (96) } \\
\text { [92; } 98]\end{array}$ & $\begin{array}{c}24 / 33(73) \\
{[60 ; 84]}\end{array}$ & $\begin{array}{c}205 / 210(98) \\
{[95 ; 99]}\end{array}$ & $\begin{array}{c}229 / 243(94) \\
{[90 ; 97]}\end{array}$ \\
\hline VNCa & $\begin{array}{c}27 / 29(93) \\
{[80 ; 99]}\end{array}$ & $\begin{array}{c}210 / 214(98) \\
{[95 ; 100]}\end{array}$ & $\begin{array}{c}27 / 31(87) \\
{[72 ; 95]}\end{array}$ & $\begin{array}{c}210 / 212(99) \\
{[97 ; 100]}\end{array}$ & $\begin{array}{c}237 / 243(98) \\
{[97 ; 100]}\end{array}$ \\
\hline
\end{tabular}

Tabelle 9: Vergleich der diagnostischen Genauigkeit aller Untersucher für die Erkennung von Affektionen von Spinalnervenwurzel pro Bandscheibe

Die Bewertung der diagnostischen Genauigkeit von Standard-CT und VNCa-Rekonstruktionen zur Detektion von Affektionen von Spinalnervenwurzeln erfolgt nach dem Bewertungssystem für die Kompression von Nervenwurzeln von Pfirmann et al. (2004) ${ }^{32}$. Die MRT diente dabei als Referenzstandard unter Berücksichtigung der Clusterbildung. Der unerfahrenste Untersucher (Untersucher 1; ein Jahr Erfahrung in MSK-Bildgebung) zeigt dabei durch die Verwendung der VNCa-Bildserien die deutlichste Verbesserung hinsichtlich der diagnostischen Genauigkeit. Untersucher 2 und 3 hatten je drei Jahre, Untersucher 4 hatte fünf Jahre, Untersucher 5 hatte sechs Jahre und Untersucher 6 hatte neun Jahre Erfahrung in der MSK-Bildgebung. 


\subsection{Qualitative Bewertung der Studienparameter}

Die Untersucher nutzten die in Tabelle 5 dargestellte Fünf-Punkte-Bewertungsskala (Kapitel 3.5).

Um die Ergebnisse grafisch zu verdeutlichen, wurden Box-and-Whisker-Plots zur Veranschaulichung herangezogen. Die Box zeigt dabei den Interquartilsabstand zwischen dem unteren Quartil (25\%-Quartil) und dem oberen Quartil (75\%-Quartil). Folglich liegen somit 50\% der Daten innerhalb der Box. Die schwarze horizontale Linie markiert in diesem Fall den Mittelwert. Von der Box ausgehend spannen sich nach oben und unten die Antennen (englisch: Whisker). Diese demonstrieren Werte die außerhalb der Box liegen. Ausreißer sind durch Punkte dargestellt und veranschaulichen die Streuung der Werte. ${ }^{140}$

Für die Beurteilung eines lumbalen Bandscheibenvorfalls zeigten die beiden Untersucher, welche die MRT-Sequenzen auswerteten, eine sehr hohe diagnostische Sicherheit. Der Mittelwert lag bei 4,85.

Eine vergleichbare Sicherheit konnte auch mithilfe der VNCa-Rekonstruktionen erreicht werden. Der Mittelwert der sechs CT-Untersucher lag hier bei 4,80 ( $P=$ 0,22). Im Gegensatz dazu zeigten diese bei Gebrauch der standardisierten CTBilder im Vergleich zu den VNCa-Bildserien einen signifikant niedrigeren Wert für die diagnostische Sicherheit (Mittelwert: 2,87) $(P<0,001)($ Abb. 11). Die Übereinstimmung zwischen den Untersuchern war sowohl für die VNCa-Rekonstruktionen, als auch für die Standard-CT „gut“ ( $\kappa=0,71$ vs. 0,61$)$. 


\section{Diagnostische Sicherheit}

\section{Score}

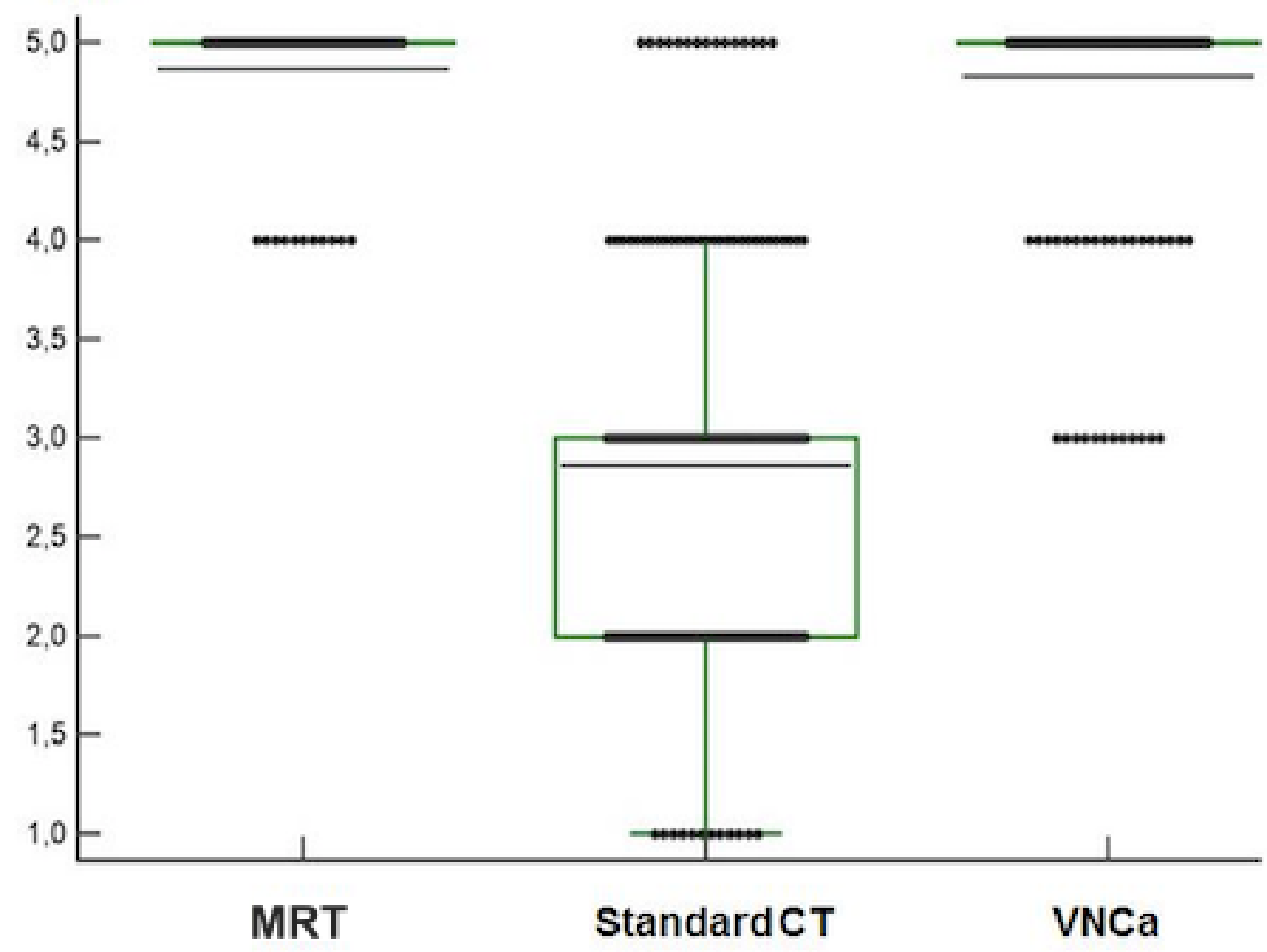

Abbildung 11: Qualitative Bewertung diagnostische Sicherheit

Mittels farbkodierter VNCa-Rekonstruktionen konnten für die diagnostische Sicherheit signifikant höhere Durchschnittswerte erzielt werden, als bei Verwendung der Standard-CT. Die schmale, schwarze horizontale Line markiert jeweils den Mittelwert (MRT: 4,37; Standard-CT: 3,06; VNCa: 4,31). Die grün markierte Box beinhaltet $50 \%$ der ermittelten Werte, die schwarzen Punkte stellen die von den Radiologen vergebenen Scores $(n=246)$ dar und demonstrieren die Streuung der Werte.

Zudem wurde das Bildrauschen evaluiert. Für die MRT-Serien errechnete sich dabei ein Mittelwert von 4,37, während sich in den VNCa-Rekonstruktionen ein Wert von 4,31 ergab. Dies zeigt ein vergleichbar niedriges Bildrauschen für die beiden Modalitäten $(P=0,26)$. Dagegen empfanden die Untersucher ein deutlich höheres Bildrauschen in der Standard-CT mit einem Mittelwert von 3,06 $(P<$ 0,001) (Abb. 12). Die Übereinstimmung zwischen den Untersuchern war dabei "exzellent“ für die VNCa-Serien $(\kappa=0,82)$ und "gut“ für die Standard-CT ( $\kappa=$ $0,74)$. 


\section{Bildrauschen}

\section{Score}

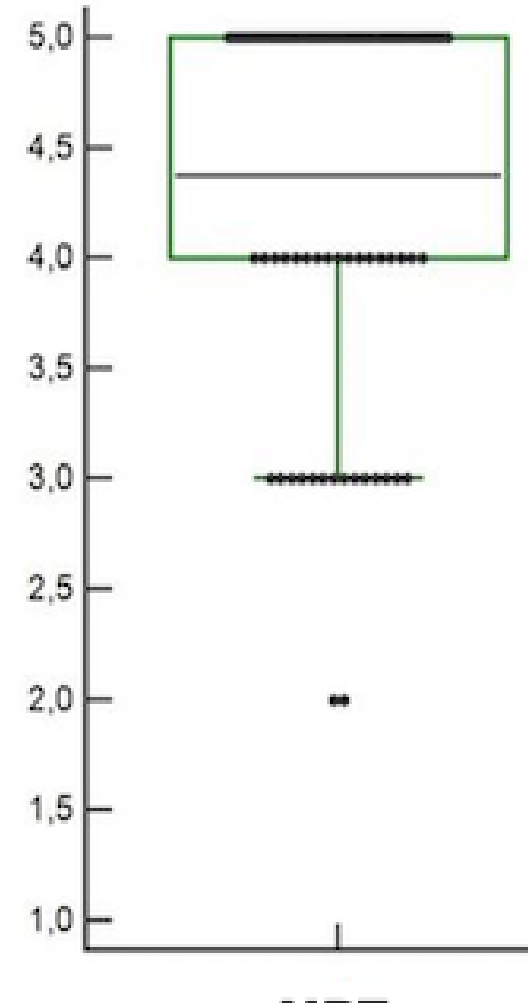

MRT

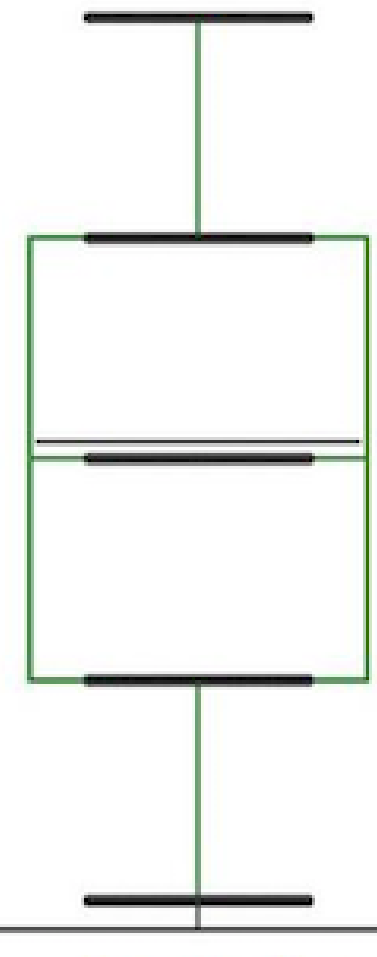

Standard CT

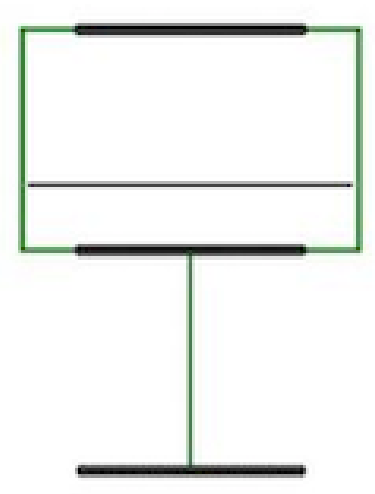

I

$\mathrm{VNCa}$

Abbildung 12: Qualitative Bewertung des Bildrauschens

Verglichen mit der Standard-CT wurde das Ausmaß des Bildrauschens von den Untersuchern bei Verwendung farbkodierter VNCa-Rekonstruktionen als signifikant geringer bewertet (Mittelwerte: Standard-CT: 3,06; VNCa: 4,31). Die Werte für die MRT-Serien implizieren ein annähernd identisches Bildrauschen von MRT und VNCa-Rekonstruktionen (Mittelwert: 4,37 vs. 4,31).

Die grün markierten Boxen beinhalten 50\% der ermittelten Werte. Die schmalen, schwarzen horizontalen Linien markieren die Mittelwerte (MRT: 4,37; Standard-CT: 3,06; VNCa: 4,31), während die schwarzen Punkte die von den Radiologen vergebenen Scores $(n=246)$ darstellen und die Streuung der Werte demonstrieren.

Des Weiteren wurde von den Untersuchern die Bildqualität beurteilt. Dabei zeigten sich nur minimale Unterschiede zwischen der MRT und den VNCa-Rekonstruktionen. Die Mittelwerte lagen bei 4,51 (MRT) und 4,48 (VNCa) $(P=0,40)$. Vergleicht man in diesem Punkt die VNCa-Rekonstruktionen mit der Standard$\mathrm{CT}$, so zeigt sich, dass die Untersucher die Bildqualität der VNCa-Bilder als signifikant besser bewerteten (Mittelwerte: 4,48 vs. 3,10) $(P<0,001)$ (Abb. 13). Die 
Übereinstimmung zwischen den Untersuchern war hierbei „exzellent“ für VNCa $(\kappa=0,81)$ und "gut" für Standard-CT $(\kappa=0,73)(P<0,001)$.

\section{Bildqualität}

\section{Score}

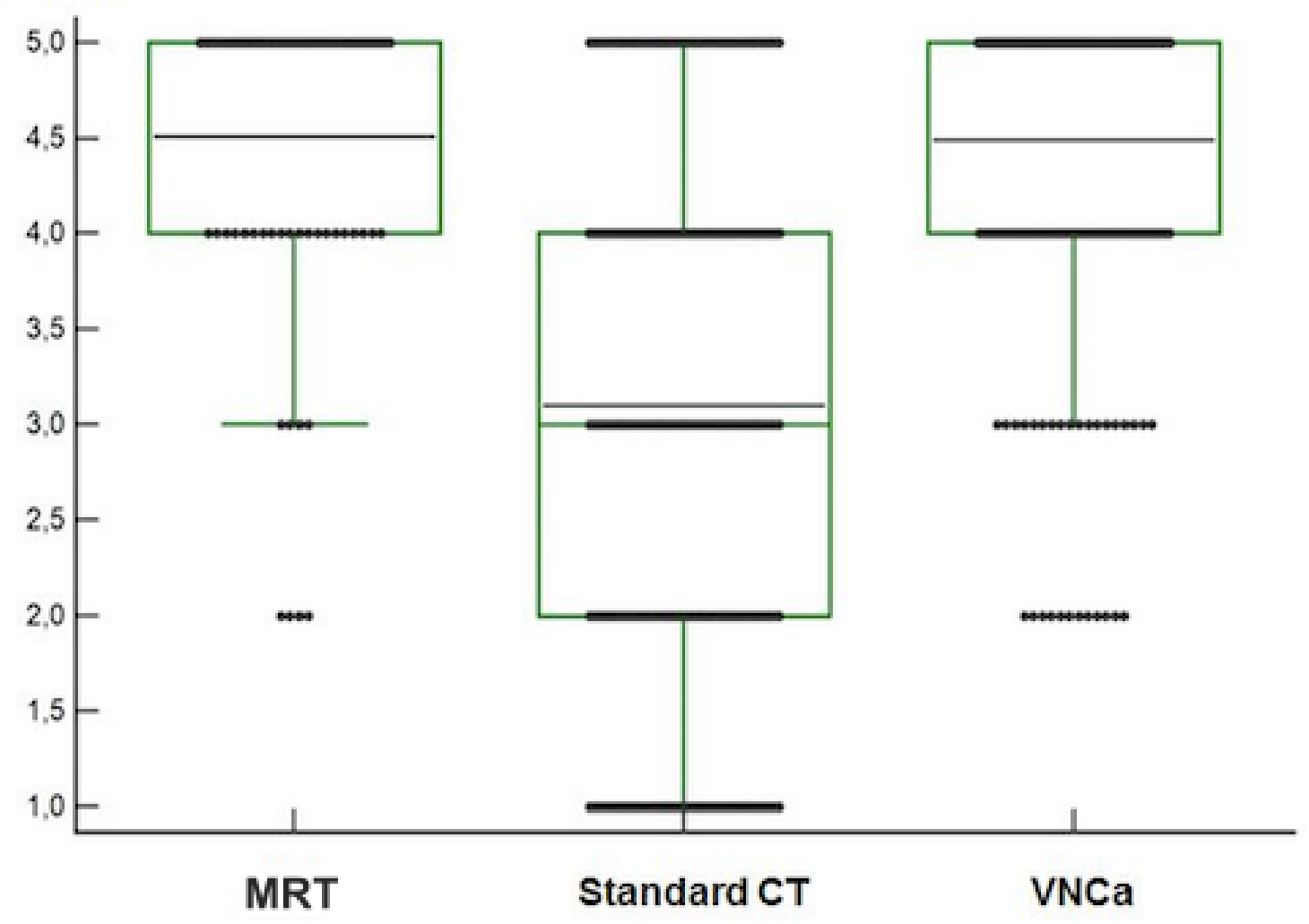

Abbildung 13: Qualitative Bewertung der Bildqualität

Bei der Beurteilung der Bildqualität zeigen die Daten nur minimale Unterschiede zwischen VNCaRekonstruktionen und der MRT, die den diagnostischen Goldstandard darstellt. Die Bildqualität der Standard-CT wurde von den Untersuchern als signifikant schlechter empfunden.

Die grün markierten Boxen repräsentieren die mittleren $50 \%$ der ermittelten Werte. Die schmalen, schwarzen horizontalen Linien markieren die Mittelwerte (MRT: 4,51; Standard-CT: 3,10; VNCa: $4,48)$ uns die schwarzen Punkte zeigen die von den Radiologen vergebenen Scores $(n=246)$ und demonstrieren damit die Streuung der Werte. 


\section{Diskussion}

In dieser Studie wurde untersucht, ob farbkodierte VNCa-Rekonstruktionen, die mittels DECT und einer entsprechenden Bildnachbearbeitungss oftware generiert wurden, einen Vorteil gegenüber der konventionellen Graustufen-CT für die Beurteilung von Bandscheibenvorfällen der Lendenwirbelsäule und der Affektion von Spinalnervenwurzeln haben.

\subsection{Interpretation der Ergebnisse}

Die Studienergebnisse zeigen, dass durch Verwendung der VNCa-Rekonstruktionen sowohl für die Darstellung einer Bandscheibenherniation als auch für die Detektion einer Beteiligung von Spinalnervenwurzeln eine wesentlich höhere diagnostische Genauigkeit erzielt werden kann, als es mithilfe der konventionellen CT möglich ist. Die statistische Auswertung der Studienparamater ergab insgesamt eine hohe Sensitivität, Spezifität, PPV und NPV zur Erkennung von lumbalen Bandscheibenvorfällen mittels farbkodierter VNCa-Rekonstruktionen. Alle Werte lagen dabei über $90 \%$. Auch bei der Beurteilung der Spinalnervenwurzeln konnten durch die Analyse der VNCa-Bilder hohe Werte erzielt werden. Mit Ausnahme des PPV (87\%) lagen auch hier alle Parameter über 90\%.

Bemerkenswert ist, dass der unerfahrenste Untersucher, der zum Zeitpunkt der Auswertung lediglich ein Jahr klinische Erfahrung in der Radiologie aufwies, die größte Verbesserung durch die Verwendung der VNCa-Rekonstruktionen erzielen konnte. Insbesondere die deutliche Steigerung der Werte für Sensitivität (101 von 112 [90\%] vs. 70 von 112 [63\%]) und PPV (101 von 114 [89\%] vs. 98 von 131 [75\%]) implizieren einen signifikanten Vorteil. Diese Verbesserung ist sehr wahrscheinlich in erster Linie auf die farbliche Darstellung zurückzuführen, die vereinfacht ausgedrückt die manuelle Fensterung der Graustufen-Bilder ersetzt und damit den Diagnoseprozess durch den verbesserten Kontrast zwischen Bandscheibe und Liquor enorm vereinfacht. Diese Feststellung lässt darauf schließen, dass insbesondere Assistenzärzte mit geringer klinischer Erfahrung von der Verwendung der VNCa-Technik profitieren können. 
Um die gesamte Breite der Studie zu bewerten, ist die Leistungsfähigkeit des erfahrensten Untersuchers ein wichtiger Indikator. Unsere Daten zeigen, dass dieser in der Lage war, in der Diagnose eines Bandscheibenvorfalls der Lendenwirbelsäule mittels der farbkodierter VNCa-Bildserien eine ähnliche hohe diagnostische Genauigkeit wie mit der MRT zu erreichen. Die Werte für Sensitivität, Spezifität, PPV und NPV lagen dabei alle bei $\geq 94 \%$.

Wichtig ist festzuhalten, dass die VNCa-Rekonstruktionen in allen untersuchten Kategorien (Diagnostische Sicherheit, Bildrauschen und Bildqualität) gegenüber der Standard-CT als überlegen bewertet wurden. Darüber hinaus erreichten die VNCa-Bilder in der Gesamtheit ähnliche Bewertungen hinsichtlich der Diagnosesicherheit, der Bildqualität und des Bildrauschens wie die MRT. Die signifikant höhere Übereinstimmung zwischen den einzelnen Untersuchern bei der Detektion von Bandscheibenherniationen und Einklemmungen von Spinalnervenwurzeln im Vergleich zur Standard-CT deutet auf eine höhere Zuverlässigkeit von VNCa-Rekonstruktionen hin. Diese Zahlen untermauern unsere Hypothese, dass farbkodierte VNCa-Bilder in diesem Anwendungsgebiet nicht nur der konventionellen CT überlegen sind, sondern auch eine valide Alternative zur MRT darstellen. 


\subsection{Klinische Relevanz}

Der Bandscheibenvorfall der Lendenwirbelsäule gilt als eine der Hauptursachen für einen akuten Schmerz im unteren Rückenbereich. ${ }^{2,66}$ Kanayama et al. (2009) konnten in ihrer Studie zeigen, dass die Prävalenz von lumbalen Bandscheibenvorfällen in Verbindung steht mit der Anzahl der Stunden, die die Patienten täglich im Sitzen verbringen. ${ }^{143}$ Bedingt durch den demographischen Wandel und den Veränderungen im Berufsleben, die häufig mit Computerarbeit und einer sitzenden Tätigkeit einhergehen, ist nicht davon auszugehen, dass sich die Zahl der Patienten in Zukunft verringern wird. Für die sichere Diagnose ist eine Schnittbildgebung unerlässlich, wobei die MRT nach aktuellem Stand als diagnostisches Verfahren der Wahl anzusehen ist. Bei Patienten mit Kontraindikationen für die Durchführung der MRT oder aufgrund anderer Umstände, die eine zeitnahe MRT-Untersuchung verhindern, bietet die CT eine entsprechende schnell verfügbare Alternative. Dabei ist hervorzuheben, dass insbesondere das Outcome von Patienten mit neurologischen Defiziten durch eine verzögerte Diagnostik und Therapie negativ beeinflusst wird. ${ }^{3}$

In vorangegangen Studien von van Rijn et al. (2012) ${ }^{9}$ und Notohamiprodjo et al. $(2017)^{10}$ zeigte die herkömmliche CT - vor allem aufgrund des niedrigen Kontrastverhältnisses zwischen Bandscheibe und Liquorflüssigkeit - nur eine moderate diagnostische Genauigkeit bei der Beurteilung von lumbalen Bandscheibenvorfällen. Eine sichere Diagnostik ist in der modernen Medizin allerdings von fundamentaler Bedeutung, da die Behandlungsstrategien in diesem Gebiet zum größten Teil auf der Zusammenschau von radiologischen und klinischen Befunden basieren.

Die Vorteile, die sich durch die Verwendung der Dual-Source- bzw. der DECT im Allgemeinen ergeben, wurden in Kapitel 2.9. umfangreich dargestellt. Für die klinische Verwendung der DECT bei lumbalen Bandscheibenvorfällen ist dabei vor allem die Möglichkeit der Bildnachbearbeitung relevant. Die DECT nutzt die Energieabhängigkeit des Absorptionsverhalten in Verbindung mit der Drei-MaterialDekomposition. Diese Kombination ermöglicht eine verbesserte Gewebequantifizierung und -differenzierung anhand der Ordnungszahl. Dies spielt insofern eine 
entscheidende Rolle für unsere Studie, da die von Pache et al. (2010) ${ }^{133}$ beschriebene VNCa-Technik, welche von uns weiter modifiziert wurde, die Darstellung von Bandscheiben realisieren kann. Dabei ist die im Verhältnis zu anderen Weichteilstrukturen relativ hohe Dichte und die Materialzusammensetzung der Bandscheibe (hoher Wassergehalt) von Vorteil. Zusätzlich wird die Visualisierung der Bandscheiben durch Verwendung unterschiedlicher CLUT weiter optimiert. Farbkodierte VNCa-Rekonstruktionen sind daher ein geeigneter Ansatz zur Verbesserung der Darstellung.

Ein weiterer Aspekt im klinischen Alltag ist die zeitliche Komponente bei der Diagnosestellung. Das Postprocessing in der vorliegenden Studie dauerte im Durchschnitt etwa vier Minuten, sodass anzunehmen ist, dass die Rekonstruktion der VNCa-Bilder aus den DECT-Datensätzen in der klinischen Routine ohne prognoseverschlechternde Zeitverzögerung anwendbar und durchführbar ist. Aus den Angaben des Statistischen Bundesamtes (Destatis) ist zu entnehmen, dass die Anzahl an verfügbaren CT-Geräten pro 100.000 Einwohner im Jahr 2017 deutlich höher war als die Dichte an MRT-Geräten (Abb. 14). ${ }^{144}$ Es ist davon auszugehen, dass diese Statistik auch heute noch repräsentativ ist.

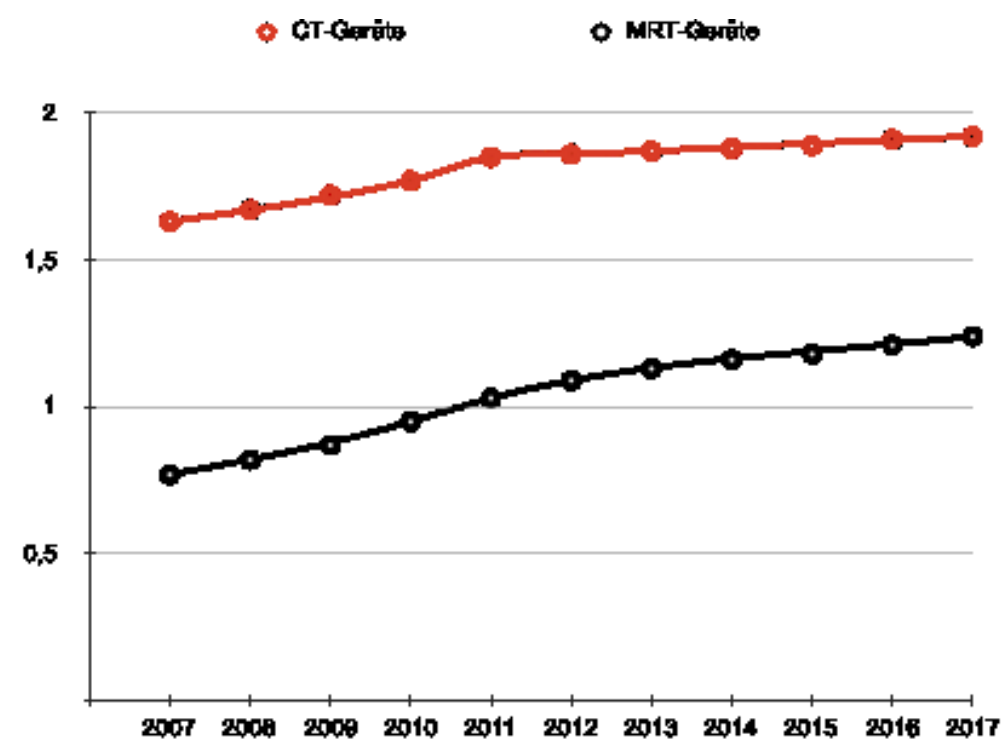

Abbildung 14: Anzahl der MRT-Geräte und CT-Geräte in deutschen Krankenhäusern

Angaben je 100.000 Einwohner, modifiziert nach Angaben des Statistischen Bundesamtes, Zweigstelle Bonn. ${ }^{144}$

Diese Daten zeigen die breitere Verfügbarkeit von CT-Geräten, wobei nicht ersichtlich ist, wie hoch der Anteil an CT-Geräten ist, die entsprechende DECTBildserien aufnehmen können. Es ist allerdings anzunehmen, dass Kliniken bei 
dem Austausch älterer CT-Geräte oder bei Neuanschaffungen vermehrt auf Computertomographen setzen, die die fortschrittliche Technik beherrschen.

Zudem konnte in mehreren Studien gezeigt werden, dass Knochenmarködeme mithilfe von VNCa-Rekonstruktionen sehr gut dargestellt werden können. ${ }^{15,133,145}$ Somit kann die DECT gegenüber der Standard-CT womöglich detailliertere kombinierte Informationen über pathologische Veränderungen der ossären Strukturen und der Bandscheiben der Lendenwirbelsäule liefern. Während die konventionelle CT in Bezug auf die morphologische Frakturdiagnostik eine akkurate und sichere Untersuchungstechnik darstellt, gilt die MRT als Goldstandard für die Beurteilung von Bandscheibenherniationen und Spinalnervenzwurzelläsionen. ${ }^{146}$ Insbesondere durch den verbesserten Nachweis von lumbalen Bandscheibenvorfällen und Affektionen von Spinalnervenwurzeln mithilfe von DECT-basierten VNCa-Rekonstruktionen wird das diagnostische Spektrum der CT somit erheblich erweitert. Durch die technische Weiterentwicklung ergibt sich damit die Möglichkeit, in lediglich einem Untersuchungsschritt einen Großteil der Erkrankungen zu diagnostizieren, die potenziell ursächlich sind für einen akuten Schmerz im unteren Rückenbereich. Dadurch kann die Zeit bis zu einer sicheren Arbeitsdiagnose im klinischen Alltag deutlich verkürzt und der Arbeitsablauf optimiert werden. Dies gilt vor allem im Falle einer eingeschränkten MRT-Verfügbarkeit (Wochenende, Nachtdienst, fehlende Ausstattung) oder bei Patienten, die Kontraindikationen für eine MRT haben wie zum Beispiel ein Cochleaimplantat, eine mechanische Herzklappe oder bestimmte Herzschrittmacher.

Zudem wird die DECT aufgrund der sehr guten Gewebedifferenzierung und den damit verbundenen vielseitigen Anwendungsmöglichkeiten immer öfter auch im Zuge sogenannter Staging-Untersuchungen im Rahmen von Tumorerkrankungen durchgeführt. ${ }^{147-150}$ Da die DECT-Untersuchungen auch retrospektiv nachverarbeitet werden können, ist damit auch bei solchen Untersuchungen im Nachgang eine optimierte farbkodierte Darstellung der Bandscheiben mittels farbkodierter VNCa-Rekonstruktionen möglich, was die Flexibilität in der klinischen Routine weiter erhöht. 


\subsection{Kritische Beurteilung der Methoden}

Eines der Hauptprobleme für die Diagnostik von lumbalen Bandscheibenvorfällen durch die CT ist der schwache Kontrast zwischen Bandscheibenmaterial und Liquor. Nach aktuellem Stand ist keine Studie bekannt, welche die Verwendung farbkodierter VNCa-Rekonstruktionen als potenziellen Lösungsansatz untersucht hat.

Die hohe diagnostische Genauigkeit für den Nachweis lumbaler Bandscheibenherniationen, welche in der vorliegenden Studie beobachtet wurde, unterstreicht, dass die DECT eine geeignete Alternative zur MRT sein kann. Die Daten deuten zudem darauf hin, dass dies auch als ein geeigneter bildgebender Ansatz zur Darstellung von Spinalnervenwurzeln denkbar ist. Im Vergleich zur konventionellen CT waren vor allem die Werte für die Sensitivität und den PPV deutlich besser.

Auffällig war, dass die Untersucher bei Verwendung der VNCa-Rekonstruktionen insbesondere Protrusionen deutlich genauer nachweisen konnten als in der Graustufen-CT. Zurückzuführen ist dies vor allem auf die farbkodierte Darstellung der lumbalen Bandscheiben und der damit verbundenen sehr guten Differenzierung zwischen Bandscheibe und Liquor.

Bei der korrekten Klassifizierung der Bandscheibenherniation konnten wir zudem beobachten, dass die Untersucher durch die VNCa-Bildserien die größte Verbesserung bei der Identifizierung von Extrusionen und Sequestrationen zeigten. Eine Abhängigkeit der Diagnostik vom BMI und damit der Patientenmorphologie konnte nicht festgestellt werden.

Wir stellten fest, dass es durch degenerative knöcherne Veränderungen zu Überlappungsproblemen zwischen ossären und Bandscheiben-bedingten Pathologien kommt, sodass es im Vergleich mit Patienten ohne Degeneration zu einer geringeren diagnostischen Genauigkeit kam. Daraus schließen wir, dass VNCaRekonstruktionen vor allem bei jüngeren Patienten, die potenziell weniger degenerative Veränderungen der lumbalen Wirbelsäule aufweisen, die höchste diagnostische Präzision bieten.

Aufgrund der sehr großen Patientenpopulation, die von Degenerationsprozessen betroffen sind, sollte diese Problematik Gegenstand zukünftiger Forschung sein. 
Als erster Ansatzpunkt dabei wäre eine Weiterentwicklung und Optimierung des angewendeten Nachbearbeitungs-Algorithmus für Patienten mit degenerativen Wirbelsäulenpathologien denkbar.

Als größter Nachteil der DECT im Vergleich zur MRT steht neben dem initial schlechteren Weichteilkontrast vor allem die Exposition des Patienten gegenüber Röntgenstrahlung im Vordergrund. Da im klinischen Alltag ein potenziell kritischer Auslöser für die Lumbalgie schnellstmöglich ausgeschlossen werden muss, ist die CT oftmals das bevorzugte diagnostische Mittel. ${ }^{9}$ Durch die technische Weiterentwicklung und die Verwendung von Dual-Source-Computertomographen der dritten Generation wird eine signifikante Reduktion der Strahlendosis erreicht. ${ }^{110,111}$ Der von uns in dieser Studie verwendete Scanner verfügt über neuartige Stellar-Detektoren, die empfindlicher sind gegenüber der Elektroneneinstrahlung. Dadurch kann die Dosiseffizienz der Untersuchung optimiert werden, was die benötigte Strahlendosis reduziert. Zu erwähnen ist auch der in der Studie bei der höheren Röhrenspannung (150 kV) verwendete Zinnfilter. Dieser schirmt den Patienten gegenüber zusätzlicher niedrigenergetischer Strahlung ab, die klinisch für den Bildeindruck nicht relevant ist. ${ }^{113}$ Gegenüber der SECT konnte in Studien gezeigt werden, dass die DECT keine höhere Strahlendosis benötigt. ${ }^{110,151}$ Es sei allerdings angemerkt, dass die benötigte Strahlendosis mit dem BMI korreliert. So werden bei adipösen Patienten deutlich höhere Strahlendosen benötigt, um ein Bild mit akzeptablem Bildrauschen zu erhalten. ${ }^{152,153}$ 


\subsection{Limitationen}

Bezüglich unserer Studie gab es einige Limitationen, die kritisch bewertet werden müssen und eine Diskussion rechtfertigen.

Als erster Punkt ist zu nennen, dass die Studienpopulation aus lediglich 41 Patienten bestand. Die Gründe hierfür sind zum einen das retrospektive Studiendesign, wodurch nur auf Daten zurückgegriffen wurde, die am Universitätsklinikum Frankfurt am Main erhoben wurden, und zum anderen die Festlegung, dass das Untersuchungsintervall zwischen der DECT- und der MRT-Untersuchung nicht länger als zwei Wochen betragen sollte, um einer möglichen zeitabhängigen Resorption der Bandscheibenvorfälle vorzubeugen. Ein multizentrischer Ansatz mit einer entsprechend größeren Patientengruppe ist erforderlich, um die Ergebnisse der vorliegenden Studie neu zu evaluieren und die Evidenz zu steigern.

Als zweiter Aspekt muss diskutiert werden, ob die Verwendung der MRT als Referenzstandard in dieser Studie für die Diagnose eines lumbalen Bandscheibenvorfalls angemessen ist. Wassenaar et al. (2012) ${ }^{154}$ zeigten in ihrer Studie, dass die MRT den Grad eines Bandscheibenvorfalls und die Beeinträchtigung der Spinalnervenwurzel möglicherweise überschätzt. In einem Review von van Rijn et al. (2012), welcher die Diagnostik von Pathologien der Wirbelsäule mittels CT evaluierte, wurden insgesamt sechs Studien untersucht, die als Referenzstandard operative Befunde wählten. Durch dieses Vorgehen wäre ein unmittelbarer Vergleich zwischen Therapie und Symptomänderung des Patienten in unserer Studie möglich gewesen.

Drittens wurden in der vorliegenden Studie zunächst die Bildserien der Graustufen-CT ausgewertet und im Anschluss daran nach acht Wochen die VNCa-Rekonstruktionen evaluiert. Alternativ wäre auch eine komplette Randomisierung der Auswertung der CT-Datensätze denkbar gewesen, um zum einen einer möglichen Erinnerungsverzerrung (Recall-Bias) vorzubeugen und zum anderen das Risiko einer statistischen Verzerrung zu minimieren.

Als vierte Einschränkung ist der unklare Einfluss von Kontrastmittel auf die VNCa-Rekonstruktion und der Bandscheibenvisualisierung zu diskutieren, da in der vorliegenden Studie lediglich kontrastmittelfreie Untersuchungen analysiert wurden. 
Als fünfte Limitation muss erwähnt werden, dass die Ergebnisse dieser Studie derzeit nur auf das herstellerspezifische DSCT-System und die dazu passende Software für die Bildnachbearbeitung anwendbar sind. Da die DECT mittlerweile auch bei Computertomographen anderer Hersteller eine etablierte Anwendungsmöglichkeit ist, ist es notwendig, durch weitere Studien die Praktikabilität und diagnostische Genauigkeit dieser Systeme in Bezug auf die Visualisierung von lumbalen Bandscheibenvorfällen im klinischen Alltag zu überprüfen. 


\subsection{Schlussfolgerung}

Ein akuter Schmerz im Bereich der Lendenwirbelsäule wird häufig durch einen Bandscheibenvorfall ausgelöst. ${ }^{1,2}$

Die VNCa-Technik, welche durch dezidiertes Postprocessing von DECT-Bildserien generiert wird, hat sich in den letzten Jahren deutlich weiterentwickelt. In der MSK-Bildgebung besitzt diese Technik großes Potenzial. ${ }^{7}$ Dabei wird sie vor allem für den Nachweis von Knochenmarködemen verwendet. ${ }^{17,19,133}$

In Hinblick auf unsere Studie sehen wir die Möglichkeit, dass durch spezielle Modifizierung der VNCa-Rekonstruktionen lumbale Bandscheibenvorfälle und assoziierte Affektionen der Spinalnervenwurzeln akkurat diagnostiziert und klassifiziert werden können. Aufgrund der exakten MRT-basierten Darstellung von Weichteilstrukturen und dem Fehlen von Röntgenstrahlung, ist die DECT aktuell nicht als gleichwertiger Ersatz zur MRT anzusehen. Dennoch gehen wir davon aus, dass die DECT eine valide Alternative für Patienten mit lumbalen Schmerzen und entsprechenden MRT-Kontraindikationen oder in Situationen eingeschränkter MRT-Verfügbarkeit darstellt.

Wir konnten mit unserer Studie zeigen, dass durch die Verwendung modifizierter farbkodierter DECT-basierter VNCa-Rekonstruktionen aus den Bilddatensätzen einer DECT die diagnostische Genauigkeit für die Detektion von lumbalen Bandscheibenvorfällen und Affektionen von Spinalnervenwurzeln im Vergleich zu konventionellen Graustufen-CT signifikant verbessert werden kann. Vor allem unerfahrene Untersucher konnten von der farbkodierten Technik am meisten profitieren und zeigten die deutlichsten Verbesserungen. Dem gegenüber war der erfahrenste Untersucher der Studie durch Anwenden der VNCa-Technik in der Lage, Bandscheibenvorfälle und Affektionen von Spinalnervenwurzeln mittels farbkodierter Rekonstruktionen ähnlich sicher zu diagnostizieren wie mithilfe der MRT, die als Goldstandard etabliert ist. Darüber hinaus war die Bildqualität und das Bildrauschen der VNCa-Bilder gleichwertig mit den MRT-Bildserien.

Zusammenfassend lässt sich festhalten, dass der von uns angewandte farbkodierte DECT-basierte VNCa-Algorithmus die diagnostische Genauigkeit bei der Beurteilung von lumbalen Bandscheibenvorfällen und assoziierten Spinalnervenwurzelaffektionen im Vergleich zur konventionellen CT signifikant verbessert. Für 
Patienten mit MRT-Komplikationen oder in Situationen eingeschränkter MRTVerfügbarkeit stellt die DECT eine valide Alternative zur MRT dar und kann die Prognose der Patienten aufgrund schnellerer akkurater radiologischer Diagnostik entsprechend positiv beeinflussen. 


\section{Literaturverzeichnis}

1. Saleem S, Aslam HM, Rehmani MA Khan, Raees A, Alvi AA, Ashraf J. Lumbar Disc Degenerative Disease: Disc Degeneration Symptoms and Magnetic Resonance Image Findings. Asian Spine J. 2013;7(4):322. doi:10.4184/asj.2013.7.4.322

2. Martin BI. Expenditures and Health Status Among Adults With Back and Neck Problems. JAMA. 2008;299(6):656. doi:10.1001/jama.299.6.656

3. Chou R, Qaseem A, Snow V, et al. Diagnosis and Treatment of Low Back Pain: A Joint Clinical Practice Guideline from the American College of Physicians and the American Pain Society. Annals of Internal Medicine. 2007;147(7):478. doi:10.7326/0003-4819-147-7-200710020-00006

4. Bečulić $H$, Skomorac R, Jusić $A$, et al. Impact of timing on surgical outcome in patients with cauda equina syndrome caused by lumbar disc herniation. MG. 2016;13(1). doi:10.17392/861-16

5. Foruria X, Ruiz de Gopegui K, García-Sánchez I, Moreta J, Aguirre U, Martínez-de los Mozos JL. Cauda equina syndrome secondary to lumbar disc herniation: Surgical delay and its relationship with prognosis. Revista Española de Cirugía Ortopédica y Traumatología (English Edition). 2016;60(3):153-159. doi:10.1016/j.recote.2016.01.007

6. Airaksinen O, Brox JI, Cedraschi C, et al. Chapter 4 European guidelines for the management of chronic nonspecific low back pain. Eur Spine J. 2006;15(S2):s192-s300. doi:10.1007/s00586-006-1072-1

7. Mallinson PI, Coupal TM, McLaughlin PD, Nicolaou S, Munk PL, Ouellette HA. Dual-Energy CT for the Musculoskeletal System. Radiology. 2016;281(3):690-707. doi:10.1148/radiol.2016151109

8. Tsai LL, Grant AK, Mortele KJ, Kung JW, Smith MP. A Practical Guide to MR Imaging Safety: What Radiologists Need to Know. RadioGraphics. 2015;35(6):1722-1737. doi:10.1148/rg.2015150108 
9. van Rijn RM, Wassenaar M, Verhagen AP, et al. Computed tomography for the diagnosis of lumbar spinal pathology in adult patients with low back pain or sciatica: a diagnostic systematic review. Eur Spine J. 2012;21(2):228-239. doi:10.1007/s00586-011-2012-2

10. Notohamiprodjo S, Stahl R, Braunagel M, et al. Diagnostic accuracy of contemporary multidetector computed tomography (MDCT) for the detection of lumbar disc herniation. European Radiology. 2017;27(8):34433451. doi:10.1007/s00330-016-4686-7

11. Nicolaou S, Yong-Hing CJ, Galea-Soler S, Hou DJ, Louis L, Munk P. Dual-Energy CT as a Potential New Diagnostic Tool in the Management of Gout in the Acute Setting. American Journal of Roentgenology. 2010;194(4):1072-1078. doi:10.2214/AJR.09.2428

12. Nicolaou S, Liang T, Murphy DT, Korzan JR, Ouellette H, Munk P. DualEnergy CT: A Promising New Technique for Assessment of the Musculoskeletal System. American Journal of Roentgenology. 2012;199:78-86. doi:10.2214/AJR.12.9117

13. Wichmann JL, Booz C, Wesarg S, et al. Dual-Energy CT-based Phantomless in Vivo Three-dimensional Bone Mineral Density Assessment of the Lumbar Spine. Radiology. 2014;271(3):778-784. doi:10.1148/radiol.13131952

14. Gruber M, Bodner G, Rath E, Supp G, Weber M, Schueller-Weidekamm C. Dual-energy computed tomography compared with ultrasound in the diagnosis of gout. Rheumatology. 2014;53(1):173-179. doi:10.1093/rheumatology/ket341

15. Petritsch B, Kosmala A, Weng AM, et al. Vertebral Compression Fractures: Third-Generation Dual-Energy CT for Detection of Bone Marrow Edema at Visual and Quantitative Analyses. Radiology. 2017;284(1):161168. doi:10.1148/radiol.2017162165 
16. Poort LJ, Stadler AAR, Ludlage JHB, Hoebers FJP, Kessler PAWH, Postma AA. Detection of Bone Marrow Edema Pattern With Dual-Energy Computed Tomography of the Pig Mandible Treated With Radiotherapy and Surgery Compared With Magnetic Resonance Imaging: Journal of Computer Assisted Tomography. 2017;41(4):553-558. doi:10.1097/RCT.0000000000000559

17. Booz C, Nöske J, Albrecht MH, et al. Traumatic bone marrow edema of the calcaneus: Evaluation of color-coded virtual non-calcium dual-energy CT in a multi-reader diagnostic accuracy study. European Journal of Radiology. 2019;118:207-214. doi:10.1016/j.ejrad.2019.07.023

18. Dareez NM, Dahlslett KH, Engesland E, Lindland ES. Scaphoid fracture: Bone marrow edema detected with dual-energy CT virtual non-calcium images and confirmed with MRI. Skeletal Radiol. 2017;46(12):1753-1756. doi:10.1007/s00256-017-2730-6

19. Booz C, Nöske J, Lenga L, et al. Color-coded virtual non-calcium dualenergy CT for the depiction of bone marrow edema in patients with acute knee trauma: a multireader diagnostic accuracy study. Eur Radiol. Published online July 26, 2019. doi:10.1007/s00330-019-06304-7

20. Wang Y, Chen Y, Zheng H, Huang X, Shan C, Bao Y. Detection of different degree traumatic vertebral bone marrow oedema by virtual non-calcium technique of dual-source dual-energy CT. Clinical Radiology. Published online November 2019:9. doi:10.1016/j.crad.2019.09.143

21. Wang $M-Y$, Zhang $X-Y, X u L$, et al. Detection of bone marrow oedema in knee joints using a dual-energy CT virtual non-calcium technique. Clinical Radiology. 2019;74(10):815.e1-815.e7. doi:10.1016/j.crad.2019.06.020

22. Chai JW, Choi J-A, Choi J-Y, Kim S, Hong SH, Kang HS. Visualization of joint and bone using dual-energy CT arthrography with contrast subtraction: in vitro feasibility study using porcine joints. Skeletal Radiol. 2014;43(5):673-678. doi:10.1007/s00256-014-1817-6 
23. Kosmala A, Weng AM, Heidemeier A, et al. Multiple Myeloma and DualEnergy CT: Diagnostic Accuracy of Virtual Noncalcium Technique for Detection of Bone Marrow Infiltration of the Spine and Pelvis. Radiology. 2018;286(1):205-213. doi:10.1148/radiol.2017170281

24. Krauss B, Grant KL, Schmidt BT, Flohr TG. The Importance of Spectral Separation: An Assessment of Dual-Energy Spectral Separation for Quantitative Ability and Dose Efficiency. Investigative Radiology. 2015;50(2):114-118. doi:10.1097/RLI.0000000000000109

25. Golob AL, Wipf JE. Low Back Pain. Medical Clinics of North America. 2014;98(3):405-428. doi:10.1016/j.mcna.2014.01.003

26. Katz JN. Lumbar Disc Disorders and Low-Back Pain: Socioeconomic Factors and Consequences. J Bone Joint Surg Am. 2006;88:21. doi:10.2106/JBJS.E. 01273

27. Mayer HM, Heider FC. Der lumbale Bandscheibenvorfall. Orthopädie und Unfallchirurgie up2date. 11(6):427-447. doi:10.1055/s-0042-105603

28. Gesundheitsberichterstattung des Bundes 19.03.2020. Krankheitskosten in Mio. € Für Deutschland. Gliederungsmerkmale: Jahre, Alter, Geschlecht, ICD10.; 2020. Accessed March 19, 2020.

29. Gesundheitsberichterstattung des Bundes 19.03.2020. Diagnosedaten Der Krankenhäuser Ab 2000 (Eckdaten Der Vollstationären Patienten Und Patientinnen). Gliederungsmerkmale: Jahre, Behandlungs-/Wohnort, ICD10.; 2020. Accessed March 19, 2020.

30. Glocker FX. Lumbale Radikulopathie, S2k-Leitlinie. Published online April 2018. Accessed March 23, 2020. www.dgn.org/leitlinien

31. Hicks GE, Morone N, Weiner DK. Degenerative Lumbar Disc and Facet Disease in Older Adults: Prevalence and Clinical Correlates. Spine. 2009;34(12):1301-1306. doi:10.1097/BRS.0b013e3181a18263

32. Pfirrmann CWA, Dora C, Schmid MR, Zanetti M, Hodler J, Boos N. MR Image-based Grading of Lumbar Nerve Root Compromise due to Disk Herniation: Reliability Study with Surgical Correlation. Radiology. 2004;230(2):583-588. doi:10.1148/radiol.2302021289 
33. Adams MA, Roughley PJ. What is Intervertebral Disc Degeneration, and What Causes It?: Spine. 2006;31(18):2151-2161. doi:10.1097/01.brs.0000231761.73859.2c

34. Dowdell J, Erwin M, Choma T, Vaccaro A, latridis J, Cho SK. Intervertebral Disk Degeneration and Repair. Neurosurgery. 2017;80(3S):S46-S54. doi:10.1093/neuros/nyw078

35. Wurzinger, Laurenz J. Duale Reihe-Anatomie. 2. überarbeitete Auflage. Georg Thieme Verlag; 2010.

36. Fardon DF, Milette PC. Nomenclature and Classification of Lumbar Disc Pathology. SPINE. 2001;26(5):E93-E113.

37. Eckstein F, Paulsen F, Tillman BN. Organe des Bewegungsapparates. In: Anatomie. Springer Berlin Heidelberg; 2010:75-307. doi:10.1007/978-3540-69483-0_4

38. Purmessur D, Cornejo MC, Cho SK, Hecht AC, latridis JC. Notochordal Cell-Derived Therapeutic Strategies for Discogenic Back Pain. Global Spine Journal. 2013;3(3):201-217. doi:10.1055/s-0033-1350053

39. Sobotta J, Welsch U, eds. Lehrbuch Histologie: Zytologie, Histologie, mikroskopische Anatomie; mit 21 Tabellen. 2. überarbeitete Auflage, 4. Nachdruck Elsevier, Urban \& Fischer; 2009.

40. Watanabe H, Yamada Y, Kimata K. Roles of Aggrecan, a Large Chondroitin Sulfate Proteoglycan, in Cartilage Structure and Function. Journal of Biochemistry. 1998;124(4):687-693. doi:10.1093/oxfordjournals.jbchem.a022166

41. Urban JPG, Smith S, Fairbank JCT. Nutrition of the Intervertebral Disc. Spine. 2004;29(23):2700-2709.

42. Paajanen $\mathrm{H}$, Lehto I, Alanen A, Erkintalo $\mathrm{M}$, Komu M. Diurnal fluid changes of lumbar discs measured indirectly by magnetic resonance imaging. J Orthop Res. 1994;12(4):509-514. doi:10.1002/jor.1100120407 
43. Linhardt $O$, Bergmann A, Bolm-Audorff $U$, et al. Die radiologische Befundung des lumbalen Bandscheibenvorfalls nach quantitativen und morphologischen Kriterien. Z Orthop Unfall. 2007;145(05):643-648. doi:10.1055/s-2007-965662

44. Krämer R, Matussek J, Theodoridis T. Bandscheibenbedingte Erkrankungen. 6. überarbeitete und aktualisierte Auflage. Georg Thieme Verlag; 2014.

45. Breitenseher J, Pones M, Breitenseher M. Nomenklatur der (lumbalen) Bandscheiben. Radiologie up2date. 2017;17(01):63-77. doi:10.1055/s0042-122606

46. Fardon DF, Williams AL, Dohring EJ, Murtagh FR, Gabriel Rothman SL, Sze GK. Lumbar disc nomenclature: version 2.0. The Spine Journal. 2014;14(11):2525-2545. doi:10.1016/j.spinee.2014.04.022

47. Konieczny MR, Reinhardt J, Prost M, Schleich C, Krauspe R. Signal Intensity of Lumbar Disc Herniations: Correlation With Age of Herniation for Extrusion, Protrusion, and Sequestration. Int $J$ Spine Surg. 2020;14(1):102-107. doi:10.14444/7014

48. Siemionow K, An H, Masuda K, Andersson G, Cs-Szabo G. The Effects of Age, Sex, Ethnicity, and Spinal Level on the Rate of Intervertebral Disc Degeneration: A Review of 1712 Intervertebral Discs. Spine. 2011;36(17):1333-1339. doi:10.1097/BRS.0b013e3181f2a177

49. Khan AN, Jacobsen HE, Khan J, et al. Inflammatory biomarkers of low back pain and disc degeneration: a review: Biomarkers of disc degeneration and back pain. Ann NY Acad Sci. 2017;1410(1):68-84. doi:10.1111/nyas.13551

50. Kadow T, Sowa G, Vo N, Kang JD. Molecular Basis of Intervertebral Disc Degeneration and Herniations: What Are the Important Translational Questions? Clin Orthop Relat Res. 2015;473(6):1903-1912. doi:10.1007/s11999-014-3774-8

51. Buckwalter JA. Aging and degeneration of the human intervertebral disc. Spine. 1995;(20):1307-1314. doi:10.1097/00007632-199506000-00022 
52. Roughley PJ. Biology of Intervertebral Disc Aging and Degeneration: Involvement of the Extracellular Matrix. Spine. 2004;29(23):2691-2699. doi:10.1097/01.brs.0000146101.53784.b1

53. Takeshima T, Kambara K, Miyata S, Ueda Y, Tamai S. Clinical and Radiographic Evaluation of Disc Excision for Lumbar Disc Herniation With and Without Posterolateral Fusion: Spine. 2000;25(4):450-456. doi:10.1097/00007632-200002150-00010

54. Benneker LM, Heini PF, Alini M, Anderson SE, Ito K. 2004 Young Investigator Award Winner: Vertebral Endplate Marrow Contact Channel Occlusions and Intervertebral Disc Degeneration: Spine. 2005;30(2):167173. doi:10.1097/01.brs.0000150833.93248.09

55. Smith LJ, Nerurkar NL, Choi K-S, Harfe BD, Elliott DM. Degeneration and regeneration of the intervertebral disc: lessons from development. Disease Models \& Mechanisms. 2011;4(1):31-41. doi:10.1242/dmm.006403

56. Varlotta GP, Brown MD, Kelsey JL, Golden AL. Familial predisposition for herniation of a lumbar disc in patients who are less than twenty-one years old:: The Journal of Bone \& Joint Surgery. 1991;73(1):124-128. doi:10.2106/00004623-199173010-00016

57. Ye S, Watts GF, Mandalia S, Humphries SE, Henney AM. Preliminary report: genetic variation in the human stromelysin promoter is associated with progression of coronary atherosclerosis. Heart. 1995;73(3):209-215. doi:10.1136/hrt.73.3.209

58. Martirosyan NL, Patel AA, Carotenuto A, et al. Genetic Alterations in Intervertebral Disc Disease. Front Surg. 2016;3. doi:10.3389/fsurg.2016.00059

59. Brayda-Bruno M, Tibiletti M, Ito K, et al. Advances in the diagnosis of degenerated lumbar discs and their possible clinical application. Eur Spine J. 2014;23(S3):315-323. doi:10.1007/s00586-013-2960-9

60. Colombier P, Clouet J, Hamel O, Lescaudron L, Guicheux J. The lumbar intervertebral disc: From embryonic development to degeneration. Joint Bone Spine. 2014;81(2):125-129. doi:10.1016/j.jbspin.2013.07.012 
61. Jakoi AM, Pannu G, D'Oro A, et al. The Clinical Correlations between Diabetes, Cigarette Smoking and Obesity on Intervertebral Degenerative Disc Disease of the Lumbar Spine. Asian Spine J. 2017;11(3):337-347. doi:10.4184/asj.2017.11.3.337

62. Elmasry S, Asfour S, de Rivero Vaccari JP, Travascio F. Effects of Tobacco Smoking on the Degeneration of the Intervertebral Disc: A Finite Element Study. Shamji M, ed. PLoS ONE. 2015;10(8):e0136137. doi:10.1371/journal.pone.0136137

63. Modic MT, Ross JS. Lumbar Degenerative Disk Disease. Radiology. 2007;245(1):43-61. doi:10.1148/radiol.2451051706

64. Li Z, Yang $\mathrm{H}$, Liu M, et al. Clinical Characteristics and Risk Factors of Recurrent Lumbar Disk Herniation: A Retrospective Analysis of Three Hundred Twenty-One Cases. SPINE. 2018;43(21):1463-1469. doi:10.1097/BRS.0000000000002655

65. Yilmaz C, Akar A, Civelek E, et al. Brucellar discitis as a cause of lumbar disc herniation: a case report. Neurologia i Neurochirurgia Polska. 2010;44(5):516-519. doi:10.1016/S0028-3843(14)60143-2

66. Amin RM, Andrade NS, Neuman BJ. Lumbar Disc Herniation. Curr Rev Musculoskelet Med. 2017;10(4):507-516. doi:10.1007/s12178-017-94414

67. Sane JC, Hope JMV, Diao S, Diouf JD, Kasse AN, Sy MH. Early presentation of traumatic cervical disc herniation with neurologic deficit and without an adjacent bone lesion. International Orthopaedics (SICOT). 2019;43(4):785-790. doi:10.1007/s00264-018-4117-2

68. Apple DF, McDonald AP, Smith RA. Identification of herniated nucleus pulposis in spinal cord injury. Spinal Cord. 1987;25(2):78-85. doi:10.1038/sc.1987.15

69. Terhaag D, Frowein RA. Traumatic disc prolapses. Neurosurg Rev. 1989;12(S1):588-594. doi:10.1007/BF01790707 
70. Casser H-R, Seddigh S, Rauschmann M. Acute Lumbar Back Pain: Investigation, Differential Diagnosis, and Treatment. Deutsches Aerzteblatt Online. Published online April 1, 2016. doi:10.3238/arztebl.2016.0223

71. Brouwers E, van de Meent H, Curt A, Starremans B, Hosman A, Bartels R. Definitions of traumatic conus medullaris and cauda equina syndrome: a systematic literature review. Spinal Cord. 2017;55(10):886-890. doi:10.1038/sc. 2017.54

72. Weinhardt C, Bergmann EG, Thorban T, Heller KD. Konus-Kauda-Syndrom. Akt Traumatol. 2005;35(1):40-42. doi:10.1055/s-2005-837547

73. Masuhr KF, Neumann M. Duale Reihe: Neurologie. 6. Auflage. Thieme Verlag; 2007.

74. Small SA, Perron AD, Brady WJ. Orthopedic pitfalls: cauda equina syndrome. The American Journal of Emergency Medicine. 2005;23(2):159163. doi:10.1016/j.ajem.2004.03.006

75. Ekedahl H, Jönsson B, Annertz M, Frobell RB. Accuracy of Clinical Tests in Detecting Disk Herniation and Nerve Root Compression in Subjects With Lumbar Radicular Symptoms. Archives of Physical Medicine and Rehabilitation. 2018;99(4):726-735. doi:10.1016/j.apmr.2017.11.006

76. Will JS, Bury DC, Miller JA. Mechanical Low Back Pain. American Family Physician. 2018;98(7):421-428.

77. Zimmer A, Reith W. Diagnostik und Therapie des BandscheibenvorfallsDiagnostics and therapy of spinal disc herniation. Radiologe. 2014;54(11):1082-1086. doi:10.1007/s00117-014-2726-3

78. Kreiner DS, Hwang SW, Easa JE, et al. An evidence-based clinical guideline for the diagnosis and treatment of lumbar disc herniation with radiculopathy. The Spine Journal. 2014;14(1):180-191. doi:10.1016/j.spinee.2013.08.003

79. Majlesi J, Togay H, Ünalan H, Toprak S. The Sensitivity and Specificity of the Slump and the Straight Leg Raising Tests in Patients With Lumbar Disc Herniation: JCR: Journal of Clinical Rheumatology. 2008;14(2):8791. doi:10.1097/RHU.0b013e31816b2f99 
80. Jensen MC, Brant-Zawadzki MN, Obuchowski N, Modic MT, Malkasian $D$, Ross JS. Magnetic Resonance Imaging of the Lumbar Spine in People without Back Pain. The New England Journal of Medicine. 1994;(331):6973. doi:10.1056/NEJM199407143310201

81. Brinjikji W, Diehn FE, Jarvik JG, et al. MRI Findings of Disc Degeneration are More Prevalent in Adults with Low Back Pain than in Asymptomatic Controls: A Systematic Review and Meta-Analysis. AJNR Am J Neuroradiol. 2015;36(12):2394-2399. doi:10.3174/ajnr.A4498

82. van Tulder M, Becker A, Bekkering T, et al. Chapter 3 European guidelines for the management of acute nonspecific low back pain in primary care. Eur Spine J. 2006;15(S2):s169-s191. doi:10.1007/s00586-0061071-2

83. Long A, Donelson R, Fung T. Does it Matter Which Exercise?: A Randomized Control Trial of Exercise for Low Back Pain. Spine. 2004;29(23):2593-2602. doi:10.1097/01.brs.0000146464.23007.2a

84. Furlan AD, Imamura M, Dryden T, Irvin E. Massage for low-back pain. In: The Cochrane Collaboration, ed. Cochrane Database of Systematic Reviews. John Wiley \& Sons, Ltd; 2008. doi:10.1002/14651858.CD001929.pub2

85. Hofstee DJ, Gijtenbeek JMM, Hoogland PH, et al. Westeinde Sciatica Trial: randomized controlled study of bed rest and physiotherapy for acute sciatica. Journal of Neurosurgery: Spine. 2002;96(1):45-49. doi:10.3171/spi.2002.96.1.0045

86. Luijsterburg PAJ, Verhagen AP, Ostelo RWJG, van Os TAG, Peul WC, Koes BW. Effectiveness of conservative treatments for the lumbosacral radicular syndrome: a systematic review. Eur Spine J. 2007;16(7):881899. doi:10.1007/s00586-007-0367-1

87. Hayden J, van Tulder MW, Malmivaara A, Koes BW. Exercise therapy for treatment of non-specific low back pain. Cochrane Back and Neck Group, ed. Cochrane Database of Systematic Reviews. Published online July 20, 2005. doi:10.1002/14651858.CD000335.pub2 
88. Hayden JA, van Tulder MW, Tomlinson G. Systematic Review: Strategies for Using Exercise Therapy To Improve Outcomes in Chronic Low Back Pain. Ann Intern Med. 2005;142(9):776. doi:10.7326/0003-4819-142-9200505030-00014

89. Wiese M, Krämer J, Becker C, Nentwig V, Theodoridis T, Teske W. Rückenschule heute. Z Orthop Unfall. 2009;147(02):194-198. doi:10.1055/s2008-1039234

90. Hilde G, Hagen K, Jamtvedt G, Winnem M. Advice to stay active as a single treatment for low-back pain and sciatica. In: The Cochrane Collaboration, ed. Cochrane Database of Systematic Reviews. John Wiley \& Sons, Ltd; 2002. doi:10.1002/14651858.CD003632

91. Hagen KB, Hilde G, Jamtvedt G, Winnem MF. The Cochrane Review of Bed Rest for Acute Low Back Pain and Sciatica: Spine. 2000;25(22):2932-2939. doi:10.1097/00007632-200011150-00016

92. Deyo RA, Weinstein JN. Low Back Pain. The New England Journal of Medicine. 2001;344(5):363-370. doi:10.1056/NEJM200102013440508

93. Oliveira CB, Maher CG, Pinto RZ, et al. Clinical practice guidelines for the management of non-specific low back pain in primary care: an updated overview. Eur Spine J. 2018;27(11):2791-2803. doi:10.1007/s00586-0185673-2

94. Urquhart DM, Hoving JL, Assendelft WJ, Roland M, van Tulder MW. Antidepressants for non-specific low back pain. Cochrane Back and Neck Group, ed. Cochrane Database of Systematic Reviews. Published online January 23, 2008. doi:10.1002/14651858.CD001703.pub3

95. Awad JN, Moskovich R. Lumbar Disc Herniations: Surgical versus Nonsurgical Treatment. Clinical Orthopaedics and Related Research. 2006;443(:):183-197. doi:10.1097/01.blo.0000198724.54891.3a

96. Manchikanti L, Abdi S, Atluri S, et al. An Update of Comprehensive Evidence-Based Guidelines for Interventional Techniques in Chronic Spinal Pain. Part II: Guidance and Recommendations. Pain Physician. Published online April 2013:49-283. 
97. Peul WC, van Houwelingen HC, van den Hout WB, et al. Surgery versus Prolonged Conservative Treatment for Sciatica. $N$ Engl $J$ Med. 2007;356(22):2245-2256. doi:10.1056/NEJMoa064039

98. Weinstein JN, Lurie JD, Tosteson TD, et al. Surgical vs Nonoperative Treatment for Lumbar Disk Herniation: The Spine Patient Outcomes Research Trial (SPORT) Observational Cohort. JAMA. 2006;296(20):2451. doi:10.1001/jama.296.20.2451

99. Chou R, Baisden J, Carragee EJ, Resnick DK, Shaffer WO, Loeser JD. Surgery for Low Back Pain: A Review of the Evidence for an American Pain Society Clinical Practice Guideline. Spine. 2009;34(10):1094-1109. doi:10.1097/BRS.0b013e3181a105fc

100. Thomé C. Chronischer Rückenschmerz - Operative Therapieansätze bei chronischen Rückenschmerzen. Anästhesiol Intensivmed Notfallmed Schmerzther. 2009;44(01):48-55. doi:10.1055/s-0028-1128186

101. Kim KY, Kim YT, Lee CS, Kang JS, Kim YJ. Magnetic resonance imaging in the evaluation of the lumbar herniated intervertebral disc. International Orthopaedics. 1993;17(4). doi:10.1007/BF00194188

102. Vogl TJ, Balzer JO, eds. Diagnostische und interventionelle Radiologie. Springer Berlin; 2011.

103. Weishaupt D, Köchli VD, Marincek B. Wie Funktioniert MRI? 7. Auflage. Springer Berlin; 2014.

104. Pabst C. Magnetresonanz-Tomographie, Lernskript für Mediziner. Published online January 2013. Accessed April 8, 2020. https://www.ukgm.de/ugm_2/deu/umr_rdi/PDF/Grundlagen_der_Magnetresonanztomographie_MRT_2013.pdf

105. Flohr T, Bruder H, Stierstorfer K, Simon J, Schaller S, Ohnesorge B. New Technical Developments in Multislice CT, Part 2: Sub-Millimeter 16-Slice Scanning and Increased Gantry Rotation Speed for Cardiac Imaging. Rofo Fortschr Geb Rontgenstr Neuen Bildgeb Verfahr. 2002;174(8):10221027. doi:10.1055/s-2002-32930 
106. Morsbach F, Desbiolles L, Raupach R, Leschka S, Schmidt B, Alkadhi H. Noise Texture Deviation: A Measure for Quantifying Artifacts in Computed Tomography Images With Iterative Reconstructions. Investigative Radiology. 2017;52(2):87-94. doi:10.1097/RLI.0000000000000312

107. Kim J-H, van Rijn RM, van Tulder MW, et al. Diagnostic accuracy of diagnostic imaging for lumbar disc herniation in adults with low back pain or sciatica is unknown; a systematic review. Chiropr Man Therap. 2018;26(1):37. doi:10.1186/s12998-018-0207-x

108. Pomerantz SR. Myelography. In: Handbook of Clinical Neurology. Vol 135. Elsevier; 2016:193-208. doi:10.1016/B978-0-444-53485-9.00010-6

109. Kalender WA, Perman WH, Vetter JR, Klotz E. Evaluation of a prototype dual-energy computed tomographic apparatus. I. Phantom studies: Dualenergy CT evaluation. I. Phantom studies. Med Phys. 1986;13(3):334339. doi:10.1118/1.595958

110. Flohr TG, McCollough $\mathrm{CH}$, Bruder $\mathrm{H}$, et al. First performance evaluation of a dual-source CT (DSCT) system. Eur Radiol. 2006;16(2):256-268. doi:10.1007/s00330-005-2919-2

111. Seidensticker PR, Seidensticker-Hofmann, eds. Dual Source Ct Imaging: With 52 Tables. Springer Medizin Heidelberg; 2008.

112. Stolzmann PDP, Gütti R. Dual-Energy-CT. In: Wie funktioniert CT?. Springer Berlin Heidelberg; 2011:165-172. doi:10.1007/978-3-642-178030_18.

113. Siemens Healthcare $\mathrm{GmbH}$. Siemens SOMATOM Force. Published online 2019.

114. Johnson TRC, Nikolaou K, Wintersperger BJ, et al. Dual-source CT cardiac imaging: initial experience. Eur Radiol. 2006;16(7):1409-1415. doi:10.1007/s00330-006-0298-y

115. Achenbach S, Ropers D, Kuettner A, et al. Contrast-enhanced coronary artery visualization by dual-source computed tomography-Initial experience. European Journal of Radiology. 2006;57(3):331-335. doi:10.1016/j.ejrad.2005.12.017 
116. Moss AJ, Williams MC, Newby DE, Nicol ED. The Updated NICE Guidelines: Cardiac CT as the First-Line Test for Coronary Artery Disease. Curr Cardiovasc Imaging Rep. 2017;10(5):15. doi:10.1007/s12410-017-94126

117. Lima JAC. Another Step Forward in CT Angiography. JACC: Cardiovascular Imaging. 2008;1(2):187-189. doi:10.1016/j.jcmg.2008.01.009

118. Baumueller S, Alkadhi H, Stolzmann P, et al. Computed Tomography of the Lung in the High-Pitch Mode: Is Breath Holding Still Required? Investigative Radiology. 2011;46(4):240-245. doi:10.1097/RLI.0b013e3181feee1a

119. Sommer WH, Schenzle JC, Becker CR, et al. Saving Dose in Triple-RuleOut Computed Tomography Examination Using a High-Pitch Dual Spiral Technique: Investigative Radiology. 2010;45(2):64-71. doi:10.1097/RLI.0b013e3181c15842

120. Hofer M. CT-Kursbuch: Ein Arbeitsbuch Für Den Einstieg in Die Computertomographie. 9. aktualisierte Auflage. Didamed Verlag; 2016.

121. Sedlmair M. Dual-Energy CT: Physikalische Modelle und Anwendungen. [Dissertation]. München: Ludwig-Maximilians-Universität. Published online 2009.

122. Macovski A, Alvarez RE, Chan JL-H, Stonestrom JP, Zatz LM. Energy dependent reconstruction in X-ray computerized tomography. Computers in Biology and Medicine. 1976;6(4):325-336. doi:10.1016/00104825(76)90069-X

123. Johnson TRC, Krauß B, Sedlmair M, et al. Material differentiation by dual energy CT: initial experience. Eur Radiol. 2007;17(6):1510-1517. doi:10.1007/s00330-006-0517-6

124. Canellas R, Digumarthy SR, Otrakji A, Kalra M. Applications of DECT in Thoracic Oncology: Evidence So Far. Clinics in Oncology. 2016; 1:1148.

125. Zhang LJ, Zhou CS, Schoepf UJ, et al. Dual-energy CT lung ventilation/perfusion imaging for diagnosing pulmonary embolism. Eur Radiol. 2013;23(10):2666-2675. doi:10.1007/s00330-013-2907-x 
126. Achenbach S, Ropers U, Kuettner A, et al. Randomized Comparison of 64-Slice Single- and Dual-Source Computed Tomography Coronary Angiography for the Detection of Coronary Artery Disease. JACC: Cardiovascular Imaging. 2008;1(2):177-186. doi:10.1016/j.jcmg.2007.11.006

127. Stehli J, Clerc OF, Fuchs TA, et al. Impact of monochromatic coronary computed tomography angiography from single-source dual-energy CT on coronary stenosis quantification. Journal of Cardiovascular Computed Tomography. 2016;10(2):135-140. doi:10.1016/j.jcct.2015.12.008

128. Albrecht MH, Trommer J, Wichmann JL, et al. Comprehensive Comparison of Virtual Monoenergetic and Linearly Blended Reconstruction Techniques in Third-Generation Dual-Source Dual-Energy Computed Tomography Angiography of the Thorax and Abdomen: Investigative Radiology. 2016;51(9):582-590. doi:10.1097/RLI.0000000000000272

129. Albrecht MH, Scholtz J-E, Hüsers K, et al. Advanced image-based virtual monoenergetic dual-energy CT angiography of the abdomen: optimization of kiloelectron volt settings to improve image contrast. Eur Radiol. 2016;26(6):1863-1870. doi:10.1007/s00330-015-3970-2

130. Fritz J, Henes J, Fuld M, Fishman E, Horger M. Dual-Energy Computed Tomography of the Knee, Ankle, and Foot: Noninvasive Diagnosis of Gout and Quantification of Monosodium Urate in Tendons and Ligaments. Semin Musculoskelet Radiol. 2016;20(01):130-136. doi:10.1055/s-00361579709

131. Bauer RW, Schulz JR, Zedler B, Graf TG, Vogl TJ. Compound analysis of gallstones using dual energy computed tomography-Results in a phantom model. European Journal of Radiology. 2010;75(1):e74-e80. doi:10.1016/j.ejrad.2009.08.004

132. Graser A, Johnson TRC, Bader M, et al. Dual Energy CT Characterization of Urinary Calculi: Initial In Vitro and Clinical Experience. Investigative Radiology. 2008;43(2):8.

133. Pache G, Krauss B, Strohm P, et al. Dual-Energy CT Virtual Noncalcium Technique: Detecting Posttraumatic Bone Marrow Lesions-Feasibility Study. Radiology. 2010;256(2):617-624. doi:10.1148/radiol.10091230 
134. Guggenberger R, Gnannt R, Hodler J, et al. Diagnostic Performance of Dual-Energy CT for the Detection of Traumatic Bone Marrow Lesions in the Ankle: Comparison with MR Imaging. Radiology. 2012;264(1):164173. doi:10.1148/radiol.12112217

135. Wang C-K, Tsai J-M, Chuang M-T, Wang M-T, Huang K-Y, Lin R-M. Bone Marrow Edema in Vertebral Compression Fractures: Detection with DualEnergy CT. Radiology. 2013;269(2):525-533. doi:10.1148/radiol.13122577

136. Bierry G, Venkatasamy A, Kremer S, Dosch J-C, Dietemann J-L. Dualenergy CT in vertebral compression fractures: performance of visual and quantitative analysis for bone marrow edema demonstration with comparison to MRI. Skeletal Radiol. 2014;43(4):485-492. doi:10.1007/s00256013-1812-3

137. Reddy T, McLaughlin PD, Mallinson PI, et al. Detection of occult, undisplaced hip fractures with a dual-energy CT algorithm targeted to detection of bone marrow edema. Emerg Radiol. 2015;22(1):25-29. doi:10.1007/s10140-014-1249-6

138. Baffour FI, Glazebrook KN, Morris JM, et al. Clinical utility of virtual noncalcium dual-energy CT in imaging of the pelvis and hip. Skeletal Radiol. 2019;48(12):1833-1842. doi:10.1007/s00256-019-03243-8

139. Liu X, Yu L, Primak AN, McCollough CH. Quantitative imaging of element composition and mass fraction using dual-energy CT: Three-material decomposition: Three-material decomposition of mass fraction. Med Phys. 2009;36(5):1602-1609. doi:10.1118/1.3097632

140. Weiß C. Basiswissen Medizinische Statistik: mit 20 Tabellen. 6., überarbeitete Auflage. Springer; 2013.

141. Genders TSS, Spronk S, Stijnen T, Steyerberg EW, Lesaffre E, Hunink MGM. Methods for Calculating Sensitivity and Specificity of Clustered Data: A Tutorial. Radiology. 2012;265(3):910-916. doi:10.1148/radiol. 12120509 
142. Landis JR, Koch GG. The Measurement of Observer Agreement for Categorical Data. Biometrics. 1977;33(1):159. doi:10.2307/2529310

143. Kanayama M, Togawa D, Takahashi C, Terai T, Hashimoto T. Cross-sectional magnetic resonance imaging study of lumbar disc degeneration in 200 healthy individuals. SPI. 2009;11(4):501-507. doi:10.3171/2009.5.SPINE08675

144. Statistisches Bundesamt, Zweigstelle Bonn. Anzahl Der Magnet-Resonanz-Geräte (MR) Und Computer-Tomographie-Geräte (CT) in Krankenhäusern Je 100.000 Einwohner Seit 1994.; 2020. Accessed April 11, 2020. http://www.gbe-bund.de/oowa921-install/servlet/oowa/aw92/dboowasys921.xwdevkit/xwd_init?gbe.isgbe-

tol/xs_start_neu/\&p_aid=i\&p_aid=54005302\&nummer=827\&p_sprache=D\&p_indsp $=50869 \& p \_$aid $=58014128$

145. Kaup M, Wichmann JL, Scholtz J-E, et al. Dual-Energy CT-based Display of Bone Marrow Edema in Osteoporotic Vertebral Compression Fractures: Impact on Diagnostic Accuracy of Radiologists with Varying Levels of Experience in Correlation to MR Imaging. Radiology. 2016;280(2):510519. doi:10.1148/radiol.2016150472

146. Prof. Dr. med. Verheyden AP, PD Dr. med. Spiegl U, Dr. med. Hölzl A. DGU-Leitlinie 012-028 Verletzungen der thorakolumbalen Wirbelsäule. Published online August 2018.

147. Sofue K, Tsurusaki M, Mileto A, et al. Dual-energy computed tomography for non-invasive staging of liver fibrosis: Accuracy of iodine density measurements from contrast-enhanced data: Staging of liver fibrosis in dualenergy CT. Hepatol Res. 2018;48(12):1008-1019. doi:10.1111/hepr.13205

148. Volterrani L, Gentili F, Fausto A, et al. Dual-Energy CT for Locoregional Staging of Breast Cancer: Preliminary Results. American Journal of Roentgenology. 2020;214(3):707-714. doi:10.2214/AJR.18.20953

149. Al-Najami I, Lahaye MJ, Beets-Tan RGH, Baatrup G. Dual-energy CT can detect malignant lymph nodes in rectal cancer. European Journal of Radiology. 2017;90:81-88. doi:10.1016/j.ejrad.2017.02.005 
150. Cheng S-M, Ling W, Zhu J, Xu J-R, Wu L-M, Gong H-X. Dual Energy Spectral CT Imaging in the assessment of Gastric Cancer and cell proliferation: A Preliminary Study. Sci Rep. 2018;8(1):17619. doi:10.1038/s41598-018-35712-w

151. Wichmann JL, Hardie A, Schopf U, et al. Single- and dual-energy CT of the abdomen: comparison of radiation dose and image quality of $2 \mathrm{nd}$ and 3rd generation dual-source CT. European Radiology. 2017;27:642-650. doi:10.1007/s00330-016-4383-6

152. Schindera ST, Nelson RC, Toth TL, et al. Effect of Patient Size on Radiation Dose for Abdominal MDCT with Automatic Tube Current Modulation: Phantom Study. American Journal of Roentgenology. 2008;190(2):W100W105. doi:10.2214/AJR.07.2891

153. Euler A, Szücs-Farkas Z, Schindera S. Möglichkeiten der Strahlenreduktion bei der CT des Körperstamms. Radiologie up2date. 2014;14(02):163176. doi:10.1055/s-0034-1365687

154. Wassenaar M, van Rijn RM, van Tulder MW, et al. Magnetic resonance imaging for diagnosing lumbar spinal pathology in adult patients with low back pain or sciatica: a diagnostic systematic review. Eur Spine J. 2012;21(2):220-227. doi:10.1007/s00586-011-2019-8 


\section{$7 \quad$ Abbildungsverzeichnis}

Abbildung 1: Formen der fokalen Herniation; modifiziert nach

Konieczny et al., $2020^{47}$ 18

Abbildung 2: Bulging von Bandscheiben; modifiziert nach

Fardon et al., $2001^{36}$ 18

Abbildung 3: Achsenbezeichnung bei der MRT 30

Abbildung 4: Darstellung eines Dual-Source-Computertomographen .33

Abbildung 5: Vektorielle Dekomposition eines Voxels, modifiziert nach

Sedlmair (2009) ${ }^{119}$

Abbildung 6: Flow-Chart zur Selektion der Studienpopulation

Abbildung 7: Bildgebung eines 65-jährigen Mannes

Abbildung 8: Bildgebung einer 62-jährigen Frau

Abbildung 9: Vergleich der Studienparameter zum Nachweis einer

lumbalen Bandscheibenherniation

Abbildung 10: Vergleich der Studienparameter zum Nachweis einer

Beteiligung von Spinalnervenwurzeln

Abbildung 11: Qualitative Bewertung diagnostische Sicherheit

Abbildung 12: Qualitative Bewertung des Bildrauschens 64

Abbildung 13: Qualitative Bewertung der Bildqualität 65

Abbildung 14: Anzahl der MRT-Geräte und CT-Geräte in deutschen Krankenhäusern. 


\section{Tabellenverzeichnis}

Tabelle 1: "Red flags" bei akuten lumbalen Rückenschmerzen, modifiziert nach Will et al. $(2018)^{75}$ und Casser et al. $(2016)^{69}$

Tabelle 2: Charakterisierung der untersuchten Patientengruppe in dieser Studie

Tabelle 3: Untersuchungseinstellungen Dual-Source-Computertomograph.....43

Tabelle 4: Daten der Magnetresonanztomographie .45

Tabelle 5: Bewertungskriterien für die Bildanalyse, modifiziert nach Notohamiprodjo et al., $2017^{10}$

Tabelle 6: Diagnostische Genauigkeit der Standard-CT und farbkodierter VNCa-Rekonstruktionen pro Patient

Tabelle 7: Diagnostische Genauigkeit von Standard-CT und farbkodierten VNCa-Rekonstruktionen zur Klassifikation von lumbalen Bandscheibenvorfällen pro Bandscheibe ..................................................56

Tabelle 8: Vergleich der diagnostischen Genauigkeit aller Untersucher zur Erkennung lumbaler Bandscheibenvorfälle pro Bandscheibe 60

Tabelle 9: Vergleich der diagnostischen Genauigkeit aller Untersucher für die Erkennung von Affektionen von Spinalnervenwurzel pro Bandscheibe 


\section{Abkürzungsverzeichnis}

\begin{tabular}{|c|c|}
\hline Abb.: & Abbildung \\
\hline AF: & Anulus fibrosus \\
\hline bzw. & beziehungsweise \\
\hline BMI: & Body-Mass-Index \\
\hline CES: & Cauda equina-Syndrom \\
\hline CLUT: & Colour Lookup Table \\
\hline CMS: & Conus medullaris-Syndrom \\
\hline CT: & Computertomographie \\
\hline DECT: & Dual-Energy-Computertomographie \\
\hline DSCT: & Dual-Source-Computertomographie \\
\hline HU: & Hounsfield-Einheiten \\
\hline ICD-10: & International Classification of Diseases \\
\hline kV: & Kilovolt \\
\hline MDCT: & Multi-Detektor-Computertomographie \\
\hline mGy: & Milligray \\
\hline MRI: & Magnetic Resonance Imaging \\
\hline MSK: & Muskuloskelettal \\
\hline MRT: & Magnetresonanztomographie \\
\hline $\mathrm{n}:$ & Anzahl \\
\hline NASS: & North American Spine Society \\
\hline NP: & Nucleus pulposus \\
\hline NPV: & Negativer prädiktiver Wert \\
\hline NSAR: & Nicht-steroidale Antirheumatika \\
\hline PACS: & Patient Archiving and Communication System \\
\hline PPV: & Positiver prädiktiver Wert \\
\hline SECT: & Single-Energy-Computertomographie \\
\hline SD: & Standardabweichung \\
\hline TIRM: & Turbo Inversion-Recovery Magnitude \\
\hline VNCa: & Virtuelle Noncalcium \\
\hline vs.: & Versus \\
\hline Z: & Ordnungszahl \\
\hline
\end{tabular}


10 Curriculum Vitae 


\section{Schriftliche Erklärung}

Ich erkläre ehrenwörtlich, dass ich die dem Fachbereich Medizin der Johann Wolfgang Goethe-Universität Frankfurt am Main zur Promotionsprüfung eingereichte Dissertation mit dem Titel

\section{Diagnostik von Bandscheibenvorfällen der Lendenwirbelsäule mittels virtueller Noncalcium Dual-Energy-Computertomographie}

am Institut für Diagnostische und Interventionelle Radiologie unter Betreuung und Anleitung von Prof. Dr. Thomas J. Vogl mit Unterstützung von Dr. Christian Booz ohne sonstige Hilfe selbst durchgeführt und bei der Abfassung der Arbeit keine anderen als die in der Dissertation angeführten Hilfsmittel benutzt habe. Darüber hinaus versichere ich, nicht die Hilfe einer kommerziellen Promotionsvermittlung in Anspruch genommen zu haben.

Ich habe bisher an keiner in- oder ausländischen Universität ein Gesuch um Zulassung zur Promotion eingereicht. Die vorliegende Arbeit wurde bisher nicht als Dissertation eingereicht.

Vorliegende Ergebnisse der Arbeit wurden in folgendem Publikationsorgan veröffentlicht:

Booz C, Nöske J, Martin SS, Albrecht MH, Yel I, Lenga L, Gruber-Roth T, Eichler K, D'Angelo T, Vogl TJ, Wichmann JL

"Virtual Noncalcium Dual-Energy CT: Detection of Lumbar Disk Herniation in Comparison with Standard Grayscale CT."

Radiology. 2019 Feb;290(2):446-455. doi:10.1148/radiol.2018181286. 